\title{
Trigonometric Fmn-Transform of Multi-Variable Functions and Its Application to the Partial Differential Equations and Image Processing
}

Robab Alikhani

Tabriz University: University of Tabriz

Irina Perfilieva ( $\square$ irina.perfilieva@osu.cz)

University of Ostrava https://orcid.org/0000-0003-1531-1111

M. Ganjeh-Alamdari

Tabriz University: University of Tabriz

\section{Research Article}

Keywords: F-transform of multi-variable functions, Trigonometric F m-transform, Fuzzy partitions, Basic functions, Approximation, Partial differential equations, Transport equation, Image processing.

Posted Date: January 5th, 2022

DOI: https://doi.org/10.21203/rs.3.rs-1096281/v1

License: (c) (i) This work is licensed under a Creative Commons Attribution 4.0 International License. Read Full License 


\title{
Trigonometric $F^{m n}$-transform of multi-variable functions and its application to the partial differential equations and image processing
}

\author{
R. Alikhani ${ }^{a}$ \\ I. Perfilieva ${ }^{b *}$ \\ M. Ganjeh-Alamdari ${ }^{a}$ \\ ${ }^{a}$ Department of Mathematics, University of Tabriz, Tabriz, Iran \\ ${ }^{b}$ University of Ostrava \\ Institute for Research and Applications of Fuzzy Modeling
}

\begin{abstract}
In this study, we focus on the extension of the trigonometric $F^{m}$-transform technique for functions with one-variable in order to improve its approximation properties at the end points of $[a, b]$ and then generalize the extended trigonometric $F^{m}$-transform technique to functions with more variables. The approximation and convergence properties of the direct and inverse multi-variable extended trigonometric $F^{m}$-transforms are discussed. The applicability of multi-variable trigonometric $F^{m}$-transforms to approximate multi-variable functions are illustrated by some examples. Moreover, some direct formulas for the multi-variable extended trigonometric $F^{m}$-transforms of partial derivatives of multi-variable functions are obtained and they are applied to solving the Cauchy problem of the transport equation. Also, the application of multi-variable extended trigonometric $F^{m}$-transforms for image compression is described. Some examples for the validity of the obtained results about the partial differential equations and image compression are given. The results are compared with some existence ones in the literature.
\end{abstract}

Keywords: F-transform of multi-variable functions, Trigonometric $F^{m}$-transform, Fuzzy partitions, Basic functions, Approximation, Partial differential equations, Transport equation, Image processing.

\section{Introduction}

The $F$-transform is a very important topic for a fuzzy modeling. The technique of the $F$ transform for functions of one-variable was first introduced by I. Perfilieva [1]. This is reflected in a number of fields such as the construction of approximate models, approximation of functions, filtering, solution of differential equations and data compression $[2,3,4,5,6]$. Initially, the $F^{m}$-transform of one-variable functions, a generalization of the $F$-transform, was introduced in

\footnotetext{
${ }^{*}$ Corresponding Author

†Irina.Perfilieva@osu.cz
} 
[7]. The $F^{m}$-transform has polynomial components of the arbitrary degree $m \geq 0$ and has some interesting approximation properties. Later, the trigonometric $F^{m}$-transform of one-variable functions ( for short ${ }^{t} F^{m}$-transform), another generalization of the $F$-transform, was developed in [8] which has trigonometric components of the arbitrary degree $m \geq 0$. The fuzzy partitions of the ${ }^{t} F^{m}$-transform are based on sinusoidal shaped functions with smoothness property. The explicit formulas for the components of the ${ }^{t} F^{m}$-transform and some interesting approximation properties of the direct and inverse ${ }^{t} F^{m}$-transform are derived there. Also, the authors showed that, if some functions are approximated by the trigonometric function, an even better approximation are obtained. The $F^{m}$-transform and ${ }^{t} F^{m}$-transform have been studied by several authors and researchers in the theoretical and applied fields $[9,10,11]$.

Some extensions of the $F$-transform for one-variable functions at the end point of the interval $[a, b]$ were suggested in some researches (see e.g. $[3,11]$ ). These extensions are an effective tool to obtain the better approximation at the end points of the interval $[a, b]$.

Some theoretical and numerical approaches have been presented for partial differential equations and image processing in literature (see e.g. $[12,13,14,15,16,17,18,19,20]$ ).

On the other hand, the $F$-transform for functions of many variables were studied in $[21,22]$. This was the starting point of the application of the $F$-transform to partial differential equations and image processing $[23,24,25]$. Also, a generalization of the $F^{1}$-transform to two-variable functions was presented in [26] and applied in image processing [27, 29].

One of the goal of this contribution is to generalize the extended ${ }^{t} F^{m}$-transform to multivariable functions (for short ${ }^{t} \bar{F}^{m n}$-transform) and obtain some approximation properties. In this research, the accuracy of the approximation by the ${ }^{t} \bar{F}^{m n}$-transform is improved to the third order, while the accuracy for the other cases of $F$-transform (former works) is of order 2. Also, the applicability of ${ }^{t} \bar{F}^{m n}$-transform is illustrated by some examples. It worth nothing that if we approximate some functions by the inverse ${ }^{t} \bar{F}^{m n}$-transform, we get an even better approximation to them in comparing with the inverse $F$-transform and $F^{1}$-transform with polynomials basis functions. Another goal is solving the Cauchy problem of the transport equation with the help of the ${ }^{t} \bar{F}^{m n}$-transform. The approximation solution obtained for the Cauchy problem of the transport equation has been compared with some existence results in the literature and it has been shown that, without any bias, the accuracy of the approximation solutions of some problems can be increased by ${ }^{t} \bar{F}^{11}$-transform. Moreover, as an example for application of the ${ }^{t} \bar{F}^{m n}$-transform on image processing, we use the trigonometric basis on compression of image. Then, we evaluate the efficiency of the ${ }^{t} \bar{F}^{m n}$-transform by comparing with the results of other cases of $F$-transform.

In this paper we have paid our attention to two-variable ${ }^{t} F^{m n}$-transform, while the definition of the extended ${ }^{t} F^{m}$-transform for multi-variable functions can be done in a similar way.

The paper is organized as follows. In Section 2, we collect and prove some basic concepts and preliminary results of one-variable fuzzy partition, $A_{k}$, and its extension, $\bar{A}_{k}$. Moreover, some theorems and definitions related to $L_{2}\left(\bar{A}_{k}\right)$ and consequently related to the direct and inverse extended ${ }^{t} F^{m}$-transforms of a function $f$ are presented. The obtained results in [8] are improved and the explicit formula for the extended ${ }^{t} F^{m}$-transform components are obtained. Moreover, some results about approximation properties of the extended ${ }^{t} F^{m}$-transform of one-variable functions are investigated which is better than the similar results in [8]. In Section 3, a fuzzy partition on the rectangle $[a, b] \times[c, d]$ from the plane and its extension are introduced which is more general than other fuzzy partitions in the literature [21, 22]. Moreover, some examples and properties of two-variable fuzzy partition are given. We construct two-variable trigonomet- 
ric basis functions by applying Gram-Schmidt procedure to the linearly independent system of trigonometric functions in Section 4 and define the direct and inverse ${ }^{t} \bar{F}^{m n}$-transform of twovariable function $f$ with trigonometric components of degree $m n$ which are generalizations of the direct and inverse ${ }^{t} F^{m}$-transform. In Section 5, some useful approximation properties of the direct and inverse ${ }^{t} \bar{F}^{m n}$-transform components of an original function have been investigated. Also, in order to illustrate the established theory of approximation properties, we report plots of the error function for some test functions. In Section 6, some direct formulas for two-variable ${ }^{t} \bar{F}^{m n}$-transform of partial derivatives of functions and their approximation properties are derived. Then, a numerical solution of the Cauchy problem for the transport equation using the ${ }^{t} \bar{F}^{m n}$-transform is investigated and some examples for the validity of our method and comparing the obtained results with some existence results in the literature are given. In Section 7, the ${ }^{t} \bar{F}^{m n}$-transform are applied for image compression. We give various examples and compare the obtained results with the other cases of $F$-transform. Finally, Appendix A is devoted to the details of the obtained results in Section 6.

\section{2 preliminary}

In this section, we present an overview of the necessary concepts and the main idea of the extended trigonometric $F^{m}$-transform (for short ${ }^{t} \bar{F}^{m}$ ) of function of one-variable which is a modified version of ${ }^{t} F^{m}$-transform stated in [8].

\subsection{Fuzzy partition and its extension of an interval}

One of the most important concepts of ${ }^{t} F^{m}$-transform is fuzzy partition stated in [8]. So, this section is devoted to the introduction of the concept of fuzzy partition. Moreover, in order to improve the approximation properties, we will state the extension of the basic function according to [3]. We consider the interval $[a, b]$ of real numbers as a common domain of all functions throughout this section.

Definition 2.1. [1] Let $x_{0}=x_{1}<\ldots<x_{p}=x_{p+1}$ be fixed nodes within $[a, b]$ such that $x_{1}=a, x_{p}=b$ and $p \geq 3$. We say that the fuzzy sets $A_{1}, \ldots, A_{p}:[a, b] \rightarrow[0,1]$, which are identified with their membership functions, constitute a fuzzy partition of $[a, b]$ if for $k=1, \ldots, p$, they fulfill the following conditions:

(1) $A_{k}:[a, b] \rightarrow[0,1], A_{k}\left(x_{k}\right)=1$.

(2) $A_{1}(x)=0$ if $x \notin\left(x_{1}, x_{2}\right), A_{p}(x)=0$ if $x \notin\left(x_{p-1}, x_{p}\right), A_{k}(x)=0$ if $x \notin\left(x_{k-1}, x_{k+1}\right)$, $k=2, \cdots, p-1$.

(3) $A_{k}(x)$ is continuous.

(4) For each $k=2, \cdots, p, A_{k}(x)$ strictly increases on $\left[x_{k-1}, x_{k}\right]$ and for $k=1, \cdots, p-1$ strictly decreases on $\left[x_{k}, x_{k+1}\right]$.

The membership functions $A_{1}, \ldots, A_{p}$ are called basic functions.

We say that the fuzzy partition $A_{1}, \ldots, A_{p}, p \geq 3$, is $h$-uniform if nodes $x_{1}, \ldots, x_{p}$ are $h$ equidistant; i.e. $x_{k}=x_{k-1}+h$ for all $k=2, \ldots, p$ where $h=\frac{b-a}{p-1}$ and the following two additional properties are fulfilled: 
(5) $A_{k}\left(x_{k}-x\right)=A_{k}\left(x_{k}+x\right)$ for all $k=2, \ldots, p-1$ and $x \in[0, h]$.

(6) $A_{k}(x)=A_{k-1}(x-h)$ and $A_{k+1}(x)=A_{k}(x-h)$ for $k=2, \ldots, p-1$ and $x \in\left[x_{k}, x_{k+1}\right]$.

The Ruspini condition on $[a, b]$ is $\sum_{k=1}^{p} A_{k}(x)=1$.

In [3], the authors have extended a h-uniform fuzzy partition by the following technique consisting of extending $[a, b]$ to $[a-h, b+h]$,

$$
\bar{A}_{1}(x)=\left\{\begin{array}{ccc}
A_{1}(2 a-x) & x \in[a-h, a], \\
A_{1}(x) & x \in[a, b] .
\end{array} \quad \bar{A}_{p}(x)=\left\{\begin{array}{cc}
A_{p}(x) & x \in[a, b], \\
A_{p}(2 b-x) & x \in[a, b+h] .
\end{array}\right.\right.
$$

Moreover, $\bar{A}_{k}(x)=A_{k}(x)$ if $k=2, \ldots, p-1$. Hereafter, we set $x_{0}=a-h$ and $x_{p+1}=b+h$.

Lemma 2.2. Consider an extended h-uniform fuzzy partition of $[a, b]$ given by basic functions $\bar{A}_{1}, \ldots, \bar{A}_{p}, p \geq 2$. Then for $k=1, \ldots, p$, we have

$$
\int_{x_{k-1}}^{x_{k+1}} \bar{A}_{k}(x) d x=h
$$

Proof. Our proof is similar to Lemma 1 in [1].

The following example is an extended h-uniform fuzzy partition which has an essential role in our study.

Example 2.3. We consider $\bar{A}_{1}, \ldots, \bar{A}_{p}$ as an extended $h$-uniform fuzzy partition by sinusoidal shaped basic functions with the following analytic representation for $k=1, \ldots, p$

$$
\bar{A}_{k}(x)=\left\{\begin{array}{cc}
\frac{1}{2}\left(\cos \frac{\pi}{h}\left(x-x_{k}\right)+1\right), & x \in\left[x_{k-1}, x_{k+1}\right], \\
0, & \text { o.w. }
\end{array}\right.
$$

These basic functions are illustrated in Fig. 1(a).

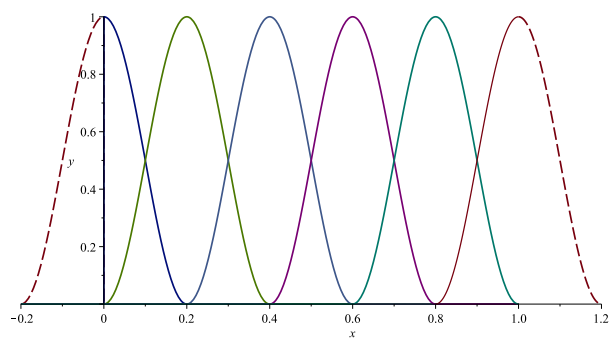

(a) Extended fuzzy partition by sinusoidal shaped basic functions

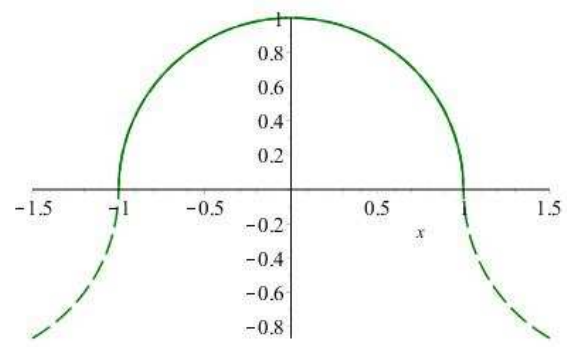

(b) Extended function for $\sqrt{1-x^{2}}$

Figure 1: Extended fuzzy partition and function. 


\subsection{Space $L_{2}\left(\bar{A}_{k}\right)$ and its subspace $L_{2}^{m}\left(\bar{A}_{k}\right)$}

In this section, we modify the linear space $L_{2}\left(A_{k}\right)$, equipped by a weighted inner product with the weight function $A_{k}$, and its subspace $L_{2}^{m}\left(A_{k}\right)$ spanned by the trigonometric basis (for more details see [8]).

Let us consider a function $f:[a, b] \rightarrow \mathbb{R}$. We can extend the function $f$ from $[a, b]$ to $[a-h, b+h]$ in different ways. As an example, the extension of the function $f(x)=\sqrt{1-x^{2}}$ from $[-1,1]$ to $[-1.5,1.5]$ is shown in Fig $1(\mathrm{~b})$. Moreover, the extension of the function $f(x)=x^{2}$ from $[-1,1]$ to $[-1.5,1.5]$ is itself. The extension of $f$ is presented by $\bar{f}$ throughout this paper.

Let $\bar{A}_{1}, \ldots, \bar{A}_{p}$ be an extended h-uniform fuzzy partition of $[a, b]$ and $k \in\{1, \ldots, p\}$. The space $L_{2}\left(\bar{A}_{k}\right)$ is a set of square-integrable functions $\bar{f}, \bar{g}:\left[x_{k-1}, x_{k+1}\right] \rightarrow \mathbb{R}$. The inner product of $\bar{f}, \bar{g} \in L_{2}\left(\bar{A}_{k}\right)$ is defined by

$$
\langle\bar{f}, \bar{g}\rangle_{k}=\frac{\int_{x_{k-1}}^{x_{k+1}} \bar{f}(x) \bar{g}(x) \bar{A}_{k}(x) d x}{\int_{x_{k-1}}^{x_{k+1}} \bar{A}_{k}(x) d x} .
$$

$L_{2}\left(\bar{A}_{k}\right)$ is a Hilbert space equipped with the norm $\|\cdot\|_{k}$ defined by $\|\bar{f}\|_{k}=\sqrt{\langle\bar{f}, \bar{f}\rangle_{k}}$.

The following lemma is a modified version of Lemmas 2 and 3 in [8].

Lemma 2.4. Let $[a, b]$ be an interval, $p \geq 2, h>0$, and $x_{0}, x_{1}, \ldots, x_{p}, x_{p+1}$ be nodes such that $x_{0}=a-h<x_{1}=a<\ldots<x_{p}=b<x_{p+1}=b+h$. Let $\bar{A}_{1}, \ldots, \bar{A}_{p}$ be an extended $h$-uniform fuzzy partition by sinusoidal shaped basic functions. Consider the set $\left\{1, \cos \frac{l \pi}{h}(x-\right.$ $\left.\left.x_{k}\right), \sin \frac{l \pi}{h}\left(x-x_{k}\right)\right\}_{l=1}^{m}$ of trigonometric functions restricted to the interval $\left[x_{k-1}, x_{k+1}\right]$ for every $k=1, \ldots, p$. Then, there exist the orthogonal trigonometric functions $u_{k}^{0}, u_{k}^{l}, v_{k}^{l}$ in $L_{2}\left(\bar{A}_{k}\right)$ as follows

$$
\begin{aligned}
& u_{k}^{0}(x)=1 \\
& u_{k}^{l}(x)=\sum_{i=1}^{l}(-1)^{l+i} \cos \frac{i \pi}{h}\left(x-x_{k}\right)+(-1)^{l} \frac{1}{2}=\cos \frac{l \pi}{h}\left(x-x_{k}\right)-u_{k}^{l-1}(x), \\
& v_{k}^{l}(x)=\sum_{i=1}^{l}(-1)^{l+i} \frac{i}{l} \sin \frac{i \pi}{h}\left(x-x_{k}\right)=\sin \frac{l \pi}{h}\left(x-x_{k}\right)-\frac{l-1}{l} v_{k}^{l-1}(x),
\end{aligned}
$$

for $l=1, \ldots, m$ and $k=1, \ldots, p$.

The orthogonal trigonometric functions $u_{k}^{0}, u_{k}^{1}, \ldots, u_{k}^{m}, v_{k}^{1}, \ldots, v_{k}^{m}$ are linearly independent. Moreover, we have for $l=1, \ldots, m, k=1, \ldots, p$

$$
\left\langle u_{k}^{0}, u_{k}^{0}\right\rangle_{k}=\langle 1,1\rangle_{k}=1, \quad\left\langle u_{k}^{l}, u_{k}^{l}\right\rangle_{k}=\frac{1}{4}, \quad\left\langle v_{k}^{l}, v_{k}^{l}\right\rangle_{k}=\frac{l+1}{4 l}
$$

and

$$
u_{k}^{l} \bar{A}_{k}=\frac{1}{4}\left(\cos \frac{(l+1) \pi}{h}\left(x-x_{k}\right)+\cos \frac{l \pi}{h}\left(x-x_{k}\right)\right), \quad v_{k}^{l} \bar{A}_{k}=\frac{1}{4}\left(\sin \frac{(l+1) \pi}{h}\left(x-x_{k}\right)+\frac{l+1}{l} \sin \frac{l \pi}{h}\left(x-x_{k}\right)\right) .
$$

Definition 2.5. Let $m \geq 0$, the linear subspace of $L_{2}\left(\bar{A}_{k}\right)$ spanned by the trigonometric basis $u_{k}^{0}, u_{k}^{1}, \ldots, u_{k}^{m}, v_{k}^{1}, \ldots, v_{k}^{m}$ is denoted by $L_{2}^{m}\left(\bar{A}_{k}\right)$.

Remark 2.6. Since we will face to fuzzy partitions on y-axis throughout this paper, we will use 
the different notations for the trigonometric basis depend on variable y as follows

$$
\begin{aligned}
\mathbf{u}_{k}^{0}(y)=1, \quad \mathbf{u}_{k}^{l}(y) & =\sum_{j=1}^{l}(-1)^{l+j} \cos \frac{j \pi}{h}\left(y-y_{k}\right)+(-1)^{l} \frac{1}{2}, \\
\mathbf{v}_{k}^{l}(y) & =\sum_{j=1}^{l}(-1)^{l+j} \frac{j}{l} \sin \frac{j \pi}{h}\left(y-y_{k}\right),
\end{aligned}
$$

for $l=1, \ldots, m$ and $k=1, \ldots, p$.

Theorem 2.7. [30] Let $H$ be a Hilbert space with the norm $\|$.$\| , and let B$ be its closed linear subspace. Then, for every element $f \in H$, there exists a unique best approximation $g_{0} \in B$ in the sense that $g_{0}$ fulfills

$$
\left\|f-g_{0}\right\|=\inf \{\|f-h\| \mid h \in B\} .
$$

Moreover, $f-g_{0} \in B^{\perp}$ and $g_{0}$ is called an orthogonal projection of $f$ on $B$.

\subsection{One-variable extended direct and inverse ${ }^{t} F^{m}$-transform}

In this section, we define the extended direct and inverse ${ }^{t} F^{m}$-transform and some their properties.

Definition 2.8. [8] Let $\bar{A}_{1}, \ldots, \bar{A}_{p}$ be extended sinusoidal shaped basic functions and $u_{k}^{0}, u_{k}^{i}, v_{k}^{i}$ $(i=1, \ldots, m)$ be the trigonometric basis of $L_{2}^{m}\left(\bar{A}_{k}\right)$. Consider $f \in L_{2}\left(\bar{A}_{k}\right)$ for $k=1, \ldots, p$. We define an extended direct ${ }^{t} F^{m}$-transform of a function $f$ with respect to $\bar{A}_{1}, \ldots, \bar{A}_{p}$ as a vector ${ }^{t} \bar{F}^{m}(f)=\left({ }^{t} \bar{F}_{1}^{m}, \ldots .{ }^{t} \bar{F}_{p}^{m}\right)$ where the $k$-th component ${ }^{t} \bar{F}_{k}^{m}$ is given by

$$
{ }^{t} \bar{F}_{k}^{m}=c_{k, 0} u_{k}^{0}+c_{k, 1} u_{k}^{1}+\ldots+c_{k, m} u_{k}^{m}+d_{k, 1} v_{k}^{1}+\ldots+d_{k, m} v_{k}^{m}
$$

where

$$
c_{k, 0}=\frac{\langle\bar{f}, 1\rangle_{k}}{\langle 1,1\rangle_{k}}=\frac{1}{2 h} \int_{x_{k-1}}^{x_{k+1}} \bar{f}(x)\left(\cos \frac{\pi}{h}\left(x-x_{k}\right)+1\right) d x,
$$

and for $i=1, \ldots, m$

$$
\begin{aligned}
& c_{k, i}=\frac{\left\langle\bar{f}, u_{k}^{i}\right\rangle_{k}}{\left\langle u_{k}^{i}, u_{k}^{i}\right\rangle_{k}}=\frac{1}{h} \int_{x_{k-1}}^{x_{k+1}} \bar{f}(x)\left(\cos \frac{(i+1) \pi}{h}\left(x-x_{k}\right)+\cos \frac{i \pi}{h}\left(x-x_{k}\right)\right) d x, \\
& d_{k, i}=\frac{\left\langle\bar{f}, v_{k}^{i}\right\rangle_{k}}{\left\langle v_{k}^{i}, v_{k}^{i}\right\rangle_{k}}=\frac{1}{h} \int_{x_{k-1}}^{x_{k+1}} \bar{f}(x)\left(\frac{i}{i+1} \sin \frac{(i+1) \pi}{h}\left(x-x_{k}\right)+\sin \frac{i \pi}{h}\left(x-x_{k}\right)\right) d x .
\end{aligned}
$$

Definition 2.9. [8] Let $\bar{A}_{1}, \ldots, \bar{A}_{p}$ be the extended sinusoidal shaped basic functions. Let $f$ : $[a, b] \rightarrow \mathbb{R}$ be a given function, $m \geq 0$, and let $\left({ }^{t} \bar{F}_{1}^{m}, \ldots,{ }^{t} \bar{F}_{p}^{m}\right)$ be the extended ${ }^{t} F^{m}$-transform of $f$. Then, the following function ${ }^{t} \bar{f}_{p}^{m}:[a-h, b+h] \rightarrow \mathbb{R}$

$$
{ }^{t} \bar{f}_{p}^{m}=\sum_{k=1}^{p}{ }^{t} \bar{F}_{k}^{m} \bar{A}_{k},
$$

is called the extended inverse ${ }^{t} F^{m}$-transform of $f$ with respect to $\left({ }^{t} \bar{F}_{1}^{m}, \ldots,{ }^{t} \bar{F}_{p}^{m}\right)$. 
In the following lemmas, we improve the similar approximation results in [8].

Lemma 2.10. Let trigonometric functions ${ }^{t} \bar{F}_{k}^{m}$ be the ${ }^{t} \bar{F}^{m}$-transform components of $f \in$ $L_{2}\left(\bar{A}_{k}\right)$. Let $\bar{f}$ be four times continuously differentiable on $[a-h, b+h]$. Then for $k=1, \ldots, p$, we have

$$
{ }^{t} \bar{F}_{k}^{m}(x)=\bar{f}(x)+O\left(h^{3}\right), \quad x \in\left[x_{k-1}, x_{k+1}\right] .
$$

Proof. We approximate $c_{k, 0}$ by trapezium formula (Euler-Maclaurin Summation Formula in [31]) with three nodes $\left\{x_{k-1}, x_{k}, x_{k+1}\right\}$ as follows

$$
c_{k, 0}=\frac{1}{2 h} \int_{x_{k-1}}^{x_{k+1}} \bar{f}(x)\left(\cos \frac{\pi}{h}\left(x-x_{k}\right)+1\right) d x=\bar{f}\left(x_{k}\right)+O\left(h^{3}\right) .
$$

In a similar way, we approximate $c_{k, i}$ for $i=1, \cdots, m$ with three nodes $\left\{x_{k-1}, x_{k}, x_{k+1}\right\}$ as follows

$$
c_{k, i}=\frac{1}{h} \int_{x_{k-1}}^{x_{k+1}} \bar{f}(x)\left(\cos \frac{(i+1) \pi}{h}\left(x-x_{k}\right)+\cos \frac{i \pi}{h}\left(x-x_{k}\right)\right) d x=O\left(h^{3}\right)
$$

Moreover, we approximate $d_{k, i}$ by trapezium formula with five nodes $\left\{x_{k-1}, x_{k}-\frac{h}{2}, x_{k}, x_{k}+\right.$ $\left.\frac{h}{2}, x_{k+1}\right\}$ and obtain

$$
d_{k, i}=\frac{1}{2}\left(\frac{i}{i+1} \sin \left(\frac{(i+1) \pi}{2}\right)+\sin \left(\frac{i \pi}{2}\right)\right)\left(\bar{f}\left(x_{k}+\frac{h}{2}\right)-\bar{f}\left(x_{k}-\frac{h}{2}\right)\right)+O\left(h^{3}\right) .
$$

By Taylor expansion, we can estimate the term $\bar{f}\left(x_{k}+\frac{h}{2}\right)-\bar{f}\left(x_{k}-\frac{h}{2}\right)$ as follows

$$
\bar{f}\left(x_{k}+\frac{h}{2}\right)-\bar{f}\left(x_{k}-\frac{h}{2}\right)=h \frac{\partial \bar{f}}{\partial x}\left(x_{k}\right)+O\left(h^{3}\right) .
$$

From Eqs. (2.1)-(2.2), we conclude

$$
d_{k, i}=\frac{1}{h} \int_{x_{k-1}}^{x_{k+1}} \bar{f}(x)\left(\frac{i}{i+1} \sin \frac{(i+1) \pi}{h}\left(x-x_{k}\right)+\sin \frac{i \pi}{h}\left(x-x_{k}\right)\right) d x=O\left(h^{3}\right) .
$$

It follows from the above equations that

$$
\begin{aligned}
{ }^{t} \bar{F}_{k}^{m}(x) & =c_{k, 0}+\sum_{i=1}^{m} c_{k, i} u_{k}^{i}(x)+\sum_{i=1}^{m} d_{k, i} v_{k}^{i}(x) \\
& =\bar{f}\left(x_{k}\right)+O\left(h^{3}\right)+O\left(h^{3}\right) \sum_{i=1}^{m} \sum_{j=1}^{i}(-1)^{i+j} \cos \frac{j \pi}{h}\left(x-x_{k}\right)+(-1)^{i} \frac{1}{2} \\
& +O\left(h^{3}\right) \sum_{i=1}^{m} \sum_{j=1}^{i}(-1)^{i+j} \frac{j}{i} \sin \frac{j \pi}{h}\left(x-x_{k}\right) \\
& =\bar{f}\left(x_{k}\right)+O\left(h^{3}\right)+O\left(h^{3}\right) \sum_{i=1}^{m} \sum_{j=1}^{i}(-1)^{i+j}\left(\sum_{n=0}^{\infty}(-1)^{n}\left(\frac{j \pi}{h}\right)^{2 n} \frac{\left(x-x_{k}\right)^{2 n}}{(2 n) !}+(-1)^{i} \frac{1}{2}\right) \\
& +O\left(h^{3}\right) \sum_{i=1}^{m} \sum_{j=1}^{i}(-1)^{i+j} \frac{j}{i}\left(\sum_{n=0}^{\infty}(-1)^{n}\left(\frac{j \pi}{h}\right)^{2 n+1} \frac{\left(x-x_{k}\right)^{2 n+1}}{(2 n+1) !}\right) \\
& =\bar{f}\left(x_{k}\right)+O\left(h^{3}\right)=\bar{f}(x)+O\left(h^{3}\right) .
\end{aligned}
$$


Lemma 2.11. Let $\bar{A}_{1}, \ldots, \bar{A}_{p}$ be the extended sinusoidal shaped basic functions and the function $\bar{f}$ be four times continuously differentiable on $[a-h, b+h]$. Let ${ }^{t} \bar{f}_{p}^{m}$ be the extended inverse ${ }^{t} F^{m}$-transform of $f$. Then, for all $x \in[a-h, b+h]$, the following estimation holds true

$$
\bar{f}(x)-{ }^{t} \bar{f}_{p}^{m}(x)=O\left(h^{3}\right) .
$$

Proof. It follows immediately from Lemma 2.10 .

\section{Fuzzy partition of a rectangle from the plane}

In this section, we focus on a generalization of the fuzzy partition for two-variable functions which the spacial case of this generalization has been presented in [21].

We will consider a rectangle $D^{2}=[a, b] \times[c, d]$ as a common domain of all real-valued functions in this section.

Definition 3.1. Let $\left(x_{k}, y_{l}\right)$ for $k=1, \ldots, p, l=1, \ldots, q$ be fixed nodes within $[a, b] \times[c, d]$ such that $a=x_{1}<\ldots<x_{p}=b, c=y_{1}<\ldots<y_{q}=d$ and $p, q \geq 2$. We say that fuzzy sets $A_{k, l}$ : $[a, b] \times[c, d] \rightarrow[0,1]$ are basic functions and constitute an fuzzy partition of $D^{2}=[a, b] \times[c, d]$ if they fulfill the following conditions for $k=1, \ldots, p, l=1, \ldots, q$.

(1) $A_{k, l}:[a, b] \times[c, d] \rightarrow[0,1], A_{k, l}\left(x_{k}, y_{l}\right)=1$.

(2) $A_{k, l}(x, y)=0$ if $(x, y) \notin\left(x_{k-1}, x_{k+1}\right) \times\left(y_{l-1}, y_{l+1}\right)$ where we let $x_{0}=a, x_{p+1}=b, y_{0}=$ $c, y_{q+1}=d$.

(3) $A_{k, l}(x, y)$ is continuous functions.

(4) For $k=2, \cdots, p, A_{k, l}(x, y)$ strictly increases with respect to $x$ on $\left[x_{k-1}, x_{k}\right]$ and for $k=$ $1, \cdots, p-1, A_{k, l}(x, y)$ strictly decreases on $\left[x_{k}, x_{k+1}\right]$ and for $l=2, \cdots, q, A_{k, l}(x, y)$ strictly increases with respect to $y$ on $\left[y_{l-1}, y_{l}\right]$ and for $l=1, \cdots, q-1, A_{k, l}(x, y)$ strictly decreases on $\left[y_{l}, y_{l+1}\right]$.

We say that the fuzzy partition $A_{k, l}$ is $h^{x} h^{y}$-uniform if $x_{k}=x_{k-1}+h^{x}, y_{l}=y_{l-1}+h^{y}$ for all $k=1, \ldots, p, l=1, \ldots, q$, where $h^{x}=\frac{b-a}{p-1}, h^{y}=\frac{d-c}{q-1}, p, q \geq 2$ and the following two additional properties are fulfilled:

(5) $A_{k, l}\left(x_{k}-x, y_{l}-y\right)=A_{k, l}\left(x_{k}+x, y_{l}+y\right)$ for $k=2, \cdots, p-1, l=2, \cdots, q-1$ and $(x, y) \in\left[0, h^{x}\right] \times\left[0, h^{y}\right]$.

(6) $A_{k, l}(x, y)=A_{k-1, l}\left(x-h^{x}, y\right)$ and $A_{k+1, l}(x, y)=A_{k, l}\left(x-h^{x}, y\right)$ for all $k=2, \cdots, p-1$ and $x \in\left[x_{k}, x_{k+1}\right]$.

$A_{k, l}(x, y)=A_{k, l-1}\left(x, y-h^{y}\right)$ and $A_{k, l+1}(x, y)=A_{k, l}\left(x, y-h^{y}\right)$ for all $l=2, \cdots, q-1$ and $y \in\left[y_{l}, y_{l+1}\right]$.

The Ruspini condition on $[a, b] \times[c, d]$ is as follows

$$
\sum_{k=1}^{p} \sum_{l=1}^{q} A_{k, l}(x, y)=1 .
$$


Hereafter, we use extended $h^{x} h^{y}$-uniform fuzzy partition and we call it extended fuzzy partition for short.

Definition 3.2. We can extend the $h^{x} h^{y}$-uniform fuzzy partition $\left[A_{k, l}\right]_{p \times q}$ to $\left[\bar{A}_{k, l}\right]_{p \times q}$ by the following technique consisting of extending $[a, b] \times[c, d]$ to $\left[a-h^{x}, b+h^{x}\right] \times\left[c-h^{y}, d+h^{y}\right]$.

$$
\begin{aligned}
& \bar{A}_{1,1}(x, y)=\left\{\begin{array}{cl}
A_{1,1}(2 a-x, 2 c-y) & (x, y) \in\left[a-h^{x}, a\right] \times\left[c-h^{y}, c\right], \\
A_{1,1}(2 a-x, y) & (x, y) \in\left[a-h^{x}, a\right] \times[c, d], \\
A_{1,1}(x, 2 c-y) & (x, y) \in[a, b] \times\left[c-h^{y}, c\right], \\
A_{1,1}(x, y) & (x, y) \in[a, b] \times[c, d] .
\end{array}\right. \\
& \bar{A}_{1, q}(x, y)=\left\{\begin{array}{cl}
A_{1, q}(2 b-x, 2 c-y) & (x, y) \in\left[b, b+h^{x}\right] \times\left[c-h^{y}, c\right], \\
A_{1, q}(2 b-x, y) & (x, y) \in\left[b, b+h^{x}\right] \times[c, d], \\
A_{1, q}(x, 2 c-y) & (x, y) \in[a, b] \times\left[c-h^{y}, c\right], \\
A_{1, q}(x, y) & (x, y) \in[a, b] \times[c, d] .
\end{array}\right. \\
& \bar{A}_{p, 1}(x, y)=\left\{\begin{array}{cl}
A_{p, 1}(2 a-x, 2 d-y) & (x, y) \in\left[a-h^{x}, a\right] \times\left[d, d+h^{y}\right], \\
A_{p, 1}(2 a-x, y) & (x, y) \in\left[a-h^{x}, a\right] \times[c, d], \\
A_{p, 1}(x, 2 d-y) & (x, y) \in[a, b] \times\left[d, d+h^{y}\right], \\
A_{p, 1}(x, y) & (x, y) \in[a, b] \times[c, d] .
\end{array}\right. \\
& \bar{A}_{p, q}(x, y)=\left\{\begin{array}{cl}
A_{p, q}(2 b-x, 2 d-y) & (x, y) \in\left[b, b+h^{x}\right] \times\left[d, d+h^{y}\right], \\
A_{p, q}(2 b-x, y) & (x, y) \in\left[b, b+h^{x}\right] \times[c, d], \\
A_{p, q}(x, 2 d-y) & (x, y) \in[a, b] \times\left[d, d+h^{y}\right], \\
A_{p, q}(x, y) & (x, y) \in[a, b] \times[c, d] .
\end{array}\right.
\end{aligned}
$$

For $l=2, \ldots, q-1$

$$
\begin{aligned}
& \bar{A}_{1, l}(x, y)=\left\{\begin{array}{cl}
A_{1, l}(x, 2 c-y) & (x, y) \in[a, b] \times\left[c-h^{y}, c\right], \\
A_{1, l}(x, y) & (x, y) \in[a, b] \times[c, d] .
\end{array}\right. \\
& \bar{A}_{p, l}(x, y)=\left\{\begin{array}{cl}
A_{p, l}(x, 2 d-y) & (x, y) \in[a, b] \times\left[d, d+h^{y}\right], \\
A_{p, l}(x, y) & (x, y) \in[a, b] \times[c, d] .
\end{array}\right.
\end{aligned}
$$

For $k=2, \ldots, p-1$

$$
\begin{aligned}
& \bar{A}_{k, 1}(x, y)=\left\{\begin{array}{cl}
A_{k, 1}(2 a-x, y) & (x, y) \in\left[a-h^{x}, a\right] \times[c, d], \\
A_{k, 1}(x, y) & (x, y) \in[a, b] \times[c, d] .
\end{array}\right. \\
& \bar{A}_{k, q}(x, y)=\left\{\begin{array}{cc}
A_{k, q}(2 b-x, y) & (x, y) \in\left[b, b+h^{x}\right] \times[c, d], \\
A_{k, q}(x, y) & (x, y) \in[a, b] \times[c, d] .
\end{array}\right.
\end{aligned}
$$

Moreover, $\bar{A}_{k, l}=A_{k, l}$ for $k=2, \ldots, p-1, l=2, \ldots, q-1$.

Remark 3.3. Based on the properties of the basic functions, $\left\{\bar{A}_{k, l}\right\}_{k(l)=1}^{p(q)}$ is equal to a shifted basic function $\bar{A}_{1,1}$, if we use uniform partition along $x$ - and $y$-axis.

Lemma 3.4. Consider $\left[\bar{A}_{k, l}\right]_{p \times q}$ as the extended fuzzy partition of $[a, b] \times[c, d]$. Let $\left(x_{k}, y_{l}\right)$ for $k=1, \cdots, p, l=1, \cdots, q$ be fixed nodes within $[a, b] \times[c, d]$ such that $a-h^{x}=x_{0}<x_{1}=a<$ $\ldots<x_{p}=b<x_{p+1}=b+h^{x}, c-h^{y}=y_{0}<y_{1}=c<\ldots<y_{q}=d<y_{q+1}=d+h^{y}$. Then for $k=1, \cdots, p, l=1, \cdots, q$, We have

$$
\int_{y_{l-1}}^{y_{l+1}} \int_{x_{k-1}}^{x_{k+1}} \bar{A}_{k, l}(x, y) d x d y=h^{x} h^{y}
$$


Proof. Since the fuzzy partition $\bar{A}_{k, l}$ is $h^{x} h^{y}$-uniform partition, we have

$$
\int_{y_{0}}^{y_{2}} \int_{x_{0}}^{x_{2}} \bar{A}_{1,1}(x, y) d x d y=\int_{y_{1}}^{y_{3}} \int_{x_{0}}^{x_{2}} \bar{A}_{1,2}(x, y) d x d y=\ldots=\int_{y_{q-1}}^{y_{q+1}} \int_{x_{p-1}}^{x_{p+1}} \bar{A}_{p, q}(x, y) d x d y .
$$

It is sufficient to prove

$$
\int_{y_{0}}^{y_{2}} \int_{x_{0}}^{x_{2}} \bar{A}_{1,1}(x, y) d x d y=h^{x} h^{y} .
$$

For this purpose, we consider $(x, y) \in\left[x_{1}, x_{2}\right] \times\left[y_{1}, y_{2}\right]$. From the Ruspini condition, we can deduce

$$
\bar{A}_{1,1}(x, y)+\bar{A}_{1,2}(x, y)+\bar{A}_{2,1}(x, y)+\bar{A}_{2,2}(x, y)=1
$$

By symmetry property of the basic functions on the rectangular $\left[x_{1}, x_{2}\right] \times\left[y_{1}, y_{2}\right]$, we have

$$
\bar{A}_{1,2}(x, y)=\bar{A}_{1,1}\left(x, y-h^{y}\right), \quad \bar{A}_{2,1}(x, y)=\bar{A}_{1,1}\left(x-h^{x}, y\right), \quad \bar{A}_{2,2}(x, y)=\bar{A}_{1,1}\left(x-h^{x}, y-h^{y}\right) .
$$

Employing the above relations and Eq. (3.1), we deduce

$$
\begin{aligned}
\int_{y_{0}}^{y_{2}} \int_{x_{0}}^{x_{2}} \bar{A}_{1,1}(x, y) d x d y & =\int_{y_{0}}^{y_{1}} \int_{x_{0}}^{x_{1}} \bar{A}_{1,1}(x, y) d x d y+\int_{y_{0}}^{y_{1}} \int_{x_{1}}^{x_{2}} \bar{A}_{1,1}(x, y) d x d y+\int_{y_{1}}^{y_{2}} \int_{x_{0}}^{x_{1}} \bar{A}_{1,1}(x, y) d x d y \\
& +\int_{y_{1}}^{y_{2}} \int_{x_{1}}^{x_{2}} \bar{A}_{1,1}(x, y) d x d y=\int_{y_{1}}^{y_{2}} \int_{x_{1}}^{x_{2}}\left(\bar{A}_{1,1}(x, y)+\bar{A}_{1,1}\left(x-h^{x}, y\right)+\bar{A}_{1,1}\left(x, y-h^{y}\right)\right. \\
& \left.+\bar{A}_{1,1}\left(x-h^{x}, y-h^{y}\right)\right) d x d y=h^{x} h^{y} .
\end{aligned}
$$

In the following, we give some examples about the extended fuzzy partition.

Example 3.5. Consider $\bar{A}_{k, l}$ as an extended fuzzy partition by basic functions with the following analytic representation which is illustrated in Fig. 2(a),

$$
\bar{A}_{k, l}(x, y)=\left\{\begin{array}{cc}
1-\left|x-x_{k}\right|, & \left|x-x_{k}\right| \leq 1,\left|y-y_{l}\right| \leq\left|x-x_{k}\right|, \\
1-\left|y-y_{l}\right|, & \left|y-y_{l}\right| \leq 1,\left|x-x_{k}\right| \leq\left|y-y_{l}\right|, \\
0 & \text { o.w. }
\end{array}\right.
$$

$\bar{A}_{k, l}$ does not satisfy the Ruspini condition.

Remark 3.6. In [21], the authors have considered $A_{k, l}(x, y)=B_{k}(x) C_{l}(y)$ where $B_{k}:[a, b] \rightarrow$ $[0,1]$ and $C_{l}:[c, d] \rightarrow[0,1]$ are basic functions and constitute a fuzzy partition of $[a, b]$ and $[c, d]$ respectively. It is the spacial case of the present fuzzy partition of $[a, b] \times[c, d]$.

Example 3.7. We consider $\bar{A}_{k, l}$ as an extended fuzzy partition by sinusoidal shaped basic functions with the following analytic representation which is illustrated in Fig. 2(b),

$$
\bar{A}_{k, l}(x, y)=\left\{\begin{array}{cc}
\frac{1}{4}\left(\cos \frac{\pi}{h^{x}}\left(x-x_{k}\right)+1\right)\left(\cos \frac{\pi}{h^{y}}\left(y-y_{l}\right)+1\right), & (x, y) \in\left[x_{k-1}, x_{k+1}\right] \times\left[y_{l-1}, y_{l+1}\right], \\
0, & \text { o.w. }
\end{array}\right.
$$

where $k=1, \ldots, p, l=1, \ldots, q$. 


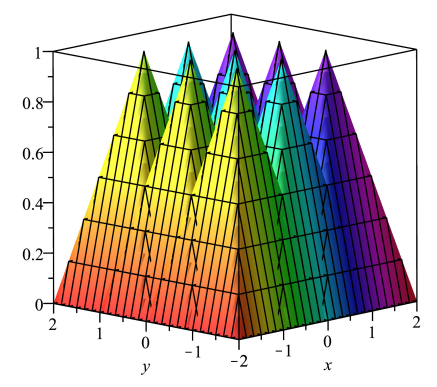

(a) Extended basic function for Example (b) Extended basic function for Ex3.5 with $m=n=2$

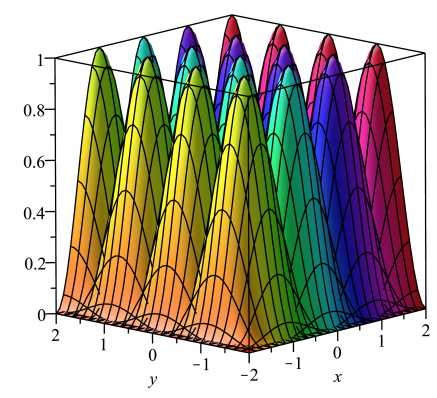

ample 3.7 with $m=n=2$

Figure 2: Extended fuzzy partition with two variable basic functions.

Definition 3.8. Let $\left[\bar{A}_{k, l}\right]_{p \times q}$ be an extended fuzzy partition of $[a, b] \times[c, d]$. Let $f \in C([a, b] \times$ $[c, d])$ and $\bar{f}$ be a continues extended of $f$ from $[a, b] \times[c, d]$ to $\left[a-h^{x}, b+h^{x}\right] \times\left[c-h^{y}, d+h^{y}\right]$. We define the direct extended $F$-transform of $f \in C([a, b] \times[c, d])$ with respect to $\bar{A}_{k, l}$ as a matrix $\bar{F}(f)=\left[\bar{F}_{k l}\right]_{p \times q}$ where the $k l$-th component $\bar{F}_{k l}$ is given by

$$
\bar{F}_{k l}=\frac{\int_{c-h^{y}}^{d+h^{y}} \int_{a-h^{x}}^{b+h^{x}} \bar{f}(x, y) \bar{A}_{k, l}(x, y) d x d y}{\int_{c-h^{y}}^{d+h^{y}} \int_{a-h^{x}}^{b+h^{x}} \bar{A}_{k, l}(x, y) d x d y}, \quad k=1, \ldots, p, l=1, \ldots, q .
$$

Definition 3.9. Let $f \in C([a, b] \times[c, d])$ and $\left[\bar{A}_{k, l}\right]_{p \times q}$ be an extended fuzzy partition of $[a, b] \times$ $[c, d]$. Let $\left[\bar{F}_{k l}\right]_{p \times q}$ be extended $F$-transform of $f$ with respect to $\bar{A}_{k, l}$. Then the function

$$
\overline{\hat{f}}_{p q}(f(x, y))=\sum_{k=1}^{p} \sum_{l=1}^{q} \bar{F}_{k l} \bar{A}_{k, l}(x, y),
$$

is called the inverse extended F-transform.

\section{Trigonometric $\bar{F}^{m n}$-transform for two-variable functions}

In this section, firstly, we introduce a space $L_{2}\left(\bar{A}_{k, l}\right)$ and its subspace. Then utilizing this space, we define the direct and inverse trigonometric $\bar{F}^{m n}$-transform of two-variable functions.

\subsection{Space $L_{2}\left(\bar{A}_{k, l}\right)$ and its subspace}

Let $\bar{A}_{k, l}$ be an extended fuzzy partition of $[a, b] \times[c, d]$ and $k \in\{1, \ldots, p\}, l \in\{1, \ldots, q\}$. The space $L_{2}\left(\bar{A}_{k, l}\right)$ is a set of square-integrable functions $\bar{f}, \bar{g}:\left[x_{k-1}, x_{k+1}\right] \times\left[y_{l-1}, y_{l+1}\right] \rightarrow \mathbb{R}$. The inner product of $\bar{f}, \bar{g} \in L_{2}\left(\bar{A}_{k, l}\right)$ is defined by

$$
\langle\bar{f}, \bar{g}\rangle_{k, l}=\frac{\int_{y_{l-1}}^{y_{l+1}} \int_{x_{k-1}}^{x_{k+1}} \bar{f}(x, y) \bar{g}(x, y) \bar{A}_{k, l}(x, y) d x d y}{\int_{y_{l-1}}^{y_{l+1}} \int_{x_{k-1}}^{x_{k+1}} \bar{A}_{k, l}(x, y) d x d y} .
$$

$L_{2}\left(\bar{A}_{k, l}\right)$ is a Hilbert space equipped with the norm $\|\cdot\|_{k, l}$ defined by $\|\bar{f}\|_{k, l}=\sqrt{\langle\bar{f}, \bar{f}\rangle_{k, l}}$.

In what follows, we are going to construct the linear subspace of $L_{2}\left(\bar{A}_{k, l}\right)$ with the extended sinusoidal 
shaped basic functions.

We consider $\bar{A}_{k, l}$ for $k=1, \ldots, p, l=1, \ldots, q$, as an extended fuzzy partition by extended sinusoidal shaped basic functions as stated in Example 3.7. For $s=1, \ldots, m, t=1, \ldots, n$, the sets $\left\{1, \cos \frac{s \pi}{h^{x}}\left(x-x_{k}\right), \cos \frac{t \pi}{h^{y}}(y-\right.$ $\left.\left.y_{l}\right), \cos \frac{s \pi}{h^{x}}\left(x-x_{k}\right) \cos \frac{t \pi}{h^{y}}\left(y-y_{l}\right)\right\},\left\{\sin \frac{s \pi}{h^{x}}\left(x-x_{k}\right) \sin \frac{t \pi}{h^{y}}\left(y-y_{l}\right)\right\},\left\{\sin \frac{t \pi}{h^{y}}\left(y-y_{l}\right), \sin \frac{t \pi}{h^{y}}\left(y-y_{l}\right) \cos \frac{s \pi}{h^{x}}\left(x-x_{k}\right)\right\}$ and $\left\{\sin \frac{s \pi}{h^{x}}\left(x-x_{k}\right), \sin \frac{s \pi}{h^{x}}\left(x-x_{k}\right) \cos \frac{t \pi}{h^{y}}\left(y-y_{l}\right)\right\}$ are linearly independent systems of trigonometric functions restricted to the interval $\left[x_{k-1}, x_{k+1}\right] \times\left[y_{l-1}, y_{l+1}\right]$. In the following lemmas, we apply the GramSchmidt process to the mentioned sets to obtain an orthogonal basis for a subspace of $L_{2}\left(\bar{A}_{k, l}\right)$.

Lemma 4.1. Let $\left(x_{k}, y_{l}\right)$ for $k=1, \ldots, p, l=1, \ldots, q,(p, q \geq 2)$ be fixed nodes within $[a, b] \times[c, d]$ such that $x_{0}=a-h^{x}, x_{k}=x_{k-1}+h^{x}$ and $y_{0}=c-h^{y}, y_{l}=y_{l-1}+h^{y}$. Let $\bar{A}_{k, l}$ be the extended fuzzy partition by the sinusoidal shaped basic functions as stated in Example 3.7.

1) Consider for $s=1, \ldots, m, t=1, \ldots, n$, the linearly independent set $\left\{1, \cos \frac{s \pi}{h^{x}}\left(x-x_{k}\right), \cos \frac{t \pi}{h^{y}}(y-\right.$ $\left.\left.y_{l}\right), \cos \frac{s \pi}{h^{x}}\left(x-x_{k}\right) \cos \frac{t \pi}{h^{y}}\left(y-y_{l}\right)\right\}$ restricted to the interval $\left[x_{k-1}, x_{k+1}\right] \times\left[y_{l-1}, y_{l+1}\right]$. Then, there exist the orthogonal trigonometric functions $\mathcal{U}_{(k, l)}^{(s, t)}(x, y)=u_{k}^{s}(x) \mathbf{u}_{l}^{t}(y)$ in $L_{2}\left(\bar{A}_{k, l}\right)$ obtained by applying the Gram-Schmidt process to the above system.

2) Consider for $s=1, \ldots, m, t=1, \ldots, n$, the linearly independent set $\left\{\sin \frac{s \pi}{h^{x}}\left(x-x_{k}\right) \sin \frac{t \pi}{h^{y}}\left(y-y_{l}\right)\right\}$ restricted to the interval $\left[x_{k-1}, x_{k+1}\right] \times\left[y_{l-1}, y_{l+1}\right]$. Then, there exist the orthogonal trigonometric functions $\mathcal{V}_{(k, l)}^{(s, t)}(x, y)=v_{k}^{s}(x) \boldsymbol{v}_{l}^{t}(y)$ in $L_{2}\left(\bar{A}_{k, l}\right)$.

3) Consider for $s=1, \ldots, m, t=1, \ldots, n$, the linearly independent set $\left\{\sin \frac{t \pi}{h^{y}}\left(y-y_{l}\right), \cos \frac{s \pi}{h^{x}}(x-\right.$ $\left.\left.x_{k}\right) \sin \frac{t \pi}{h^{y}}\left(y-y_{l}\right)\right\}$ restricted to the interval $\left[x_{k-1}, x_{k+1}\right] \times\left[y_{l-1}, y_{l+1}\right]$. Then, there exist the orthogonal trigonometric functions $\mathcal{X}_{(k, l)}^{(s, t)}(x, y)=u_{k}^{s}(x) \boldsymbol{v}_{l}^{t}(y)$ in $L_{2}\left(\bar{A}_{k, l}\right)$.

4) Consider for $s=1, \ldots, m, t=1, \ldots, n$, the linearly independent set $\left\{\sin \frac{s \pi}{h^{x}}\left(x-x_{k}\right), \sin \frac{s \pi}{h^{x}}(x-\right.$ $\left.\left.x_{k}\right) \cos \frac{t \pi}{h^{y}}\left(y-y_{l}\right)\right\}$ restricted to the interval $\left[x_{k-1}, x_{k+1}\right] \times\left[y_{l-1}, y_{l+1}\right]$. Then, there exist the orthogonal trigonometric functions $\mathcal{Y}_{(k, l)}^{(s, t)}(x, y)=v_{k}^{s}(x) \boldsymbol{u}_{l}^{t}(y)$ in $L_{2}\left(\bar{A}_{k, l}\right)$.

Proof. Case 1. Applying the Gram-Schmidt process to the system $\left\{1, \cos \frac{s \pi}{h^{x}}\left(x-x_{k}\right), \cos \frac{t \pi}{h^{y}}\left(y-y_{l}\right), \cos \frac{s \pi}{h^{x}}(x-\right.$ $\left.\left.x_{k}\right) \cos \frac{t \pi}{h^{y}}\left(y-y_{l}\right)\right\}$, we gain the orthogonal trigonometric functions $\mathcal{U}_{(k, l)}^{(s, t)}$ by the following recursive equations

$\mathcal{U}_{(k, l)}^{(0,0)}(x, y)=1$ and for $s=1, \ldots, m, t=1, \ldots, n$

$$
\begin{gathered}
\mathcal{U}_{(k, l)}^{(s, 0)}(x, y)=\cos \frac{s \pi}{h^{x}}\left(x-x_{k}\right)-\sum_{i=0}^{s-1} \lambda_{i, 0}^{(s, 0)} \mathcal{U}_{(k, l)}^{(i, 0)}(x, y), \\
\mathcal{U}_{(k, l)}^{(0, t)}(x, y)=\cos \frac{t \pi}{h^{y}}\left(y-y_{l}\right)-\sum_{j=0}^{t-1} \lambda_{0, j}^{(0, t)} \mathcal{U}_{(k, l)}^{(0, j)}(x, y), \\
\mathcal{U}_{(k, l)}^{(s, t)}(x, y)=\cos \frac{s \pi}{h^{x}}\left(x-x_{k}\right) \cos \frac{t \pi}{h^{y}}\left(y-y_{l}\right)-\sum_{j=0}^{t-1} \lambda_{s, j}^{(s, t)} \mathcal{U}_{(k, l)}^{(s, j)}(x, y)-\sum_{i=0}^{s-1} \lambda_{i, t}^{(s, t)} \mathcal{U}_{(k, l)}^{(i, t)}(x, y) \\
-\sum_{i=0}^{s-1} \sum_{j=0}^{t-1} \lambda_{i, j}^{(s, t)} \mathcal{U}_{(k, l)}^{(i, j)}(x, y),
\end{gathered}
$$

where

$$
\left.\lambda_{i, j}^{(s, t)}=\frac{\left\langle\cos \frac{s \pi}{h^{x}}\left(x-x_{k}\right) \cos \frac{t \pi}{h^{y}}\left(y-y_{l}\right), \mathcal{U}_{(k, l)}^{(i, j)}(x, y)\right\rangle_{k, l}}{\left\langle\mathcal{U}_{(k, l)}^{(i, j)}(x, y), \mathcal{U}_{(k, l)}^{(i, j)}(x, y)\right\rangle_{k, l}}, \quad i=0, \ldots, s, j=0, \ldots, t \quad \text { (except } \mathrm{i}=\mathrm{s}, \mathrm{j}=\mathrm{t}\right) .
$$


We use the double mathematics induction in order to prove $\mathcal{U}_{(k, l)}^{(s, t)}(x, y)=u_{k}^{s}(x) \mathbf{u}_{l}^{t}(y)$ for $s=0, \ldots, m$, $t=0, \ldots, n$. From Lemma 2.4 , it is clear that

$$
\mathcal{U}_{(k, l)}^{(0,0)}(x, y)=1=u_{k}^{0}(x) \mathbf{u}_{l}^{0}(y) .
$$

We consider the following mathematics induction hypothesis on $s$

$$
\mathcal{U}_{(k, l)}^{(s, 0)}(x, y)=u_{k}^{s}(x) \mathbf{u}_{l}^{0}(y) .
$$

We conclude

$$
\begin{aligned}
& \mathcal{U}_{(k, l)}^{(s+1,0)}(x, y)=\cos \frac{(s+1) \pi}{h^{x}}\left(x-x_{k}\right)-\sum_{i=0}^{s} \lambda_{i, 0}^{(s+1,0)} \mathcal{U}_{(k, l)}^{(i, 0)}(x, y) \\
& =\cos \frac{(s+1) \pi}{h^{x}}\left(x-x_{k}\right)-\sum_{i=0}^{s} \frac{\left\langle\cos \frac{(s+1) \pi}{h^{x}}\left(x-x_{k}\right), u_{k}^{i}(x)\right\rangle_{k}}{\left\langle u_{k}^{i}(x), u_{k}^{i}(x)\right\rangle_{k}} u_{k}^{i}(x) \\
& =\cos \frac{(s+1) \pi}{h^{x}}\left(x-x_{k}\right)-u_{k}^{s}(x)=u_{k}^{s+1}(x) \mathbf{u}_{l}^{0}(y) .
\end{aligned}
$$

In a similar way, we have $\mathcal{U}_{(k, l)}^{(0, t)}(x, y)=u_{k}^{0}(x) \mathbf{u}_{l}^{t}(y)$ for $t \in \mathbb{N} \cup 0$. Now we use the double mathematics induction in order to prove $\mathcal{U}_{(k, l)}^{(s, t)}(x, y)=u_{k}^{s}(x) \mathbf{u}_{l}^{t}(y)$. Therefore, we assume $\mathcal{U}_{(k, l)}^{(s+1, t)}(x, y)=u_{k}^{s+1}(x) \mathbf{u}_{l}^{t}(y)$ and $\mathcal{U}_{(k, l)}^{(s, t+1)}(x, y)=u_{k}^{s}(x) \mathbf{u}_{l}^{t+1}(y)$ for some $s, t \in \mathbb{N} \cup\{0\}$. Thus, we will conclude $\mathcal{U}_{(k, l)}^{(s+1, t+1)}(x, y)=$ $u_{k}^{s+1}(x) \mathbf{u}_{l}^{t+1}(y)$. It follows from Eq. (4.1) that

$$
\begin{aligned}
& \mathcal{U}_{(k, l)}^{(s+1, t+1)}(x, y)=\cos \frac{(s+1) \pi}{h^{x}}\left(x-x_{k}\right) \cos \frac{(t+1) \pi}{h^{y}}\left(y-y_{l}\right)-\sum_{j=0}^{t} \lambda_{s+1, j}^{(s+1, t+1)} u_{k}^{s+1}(x) \mathbf{u}_{l}^{j}(y) \\
& -\sum_{i=0}^{s} \lambda_{i, t+1}^{(s+1, t+1)} u_{k}^{i}(x) \mathbf{u}_{l}^{t+1}(y)-\sum_{i=0}^{s} \sum_{j=0}^{t} \lambda_{i, j}^{(s+1, t+1)} u_{k}^{i}(x) \mathbf{u}_{l}^{j}(y) \\
& =\cos \frac{(s+1) \pi}{h^{x}}\left(x-x_{k}\right) \cos \frac{(t+1) \pi}{h^{y}}\left(y-y_{l}\right) \\
& -\frac{\left\langle\cos \frac{(s+1) \pi}{h^{x}}\left(x-x_{k}\right), u_{k}^{s+1}(x)\right\rangle_{k}}{\left\langle u_{k}^{s+1}(x), u_{k}^{s+1}(x)\right\rangle_{k}} u_{k}^{s+1}(x) \sum_{j=0}^{t} \frac{\left\langle\cos \frac{(t+1) \pi}{h^{y}}\left(y-y_{l}\right), \mathbf{u}_{l}^{j}(y)\right\rangle_{l}}{\left\langle\mathbf{u}_{l}^{j}(y), \mathbf{u}_{l}^{j}(y)\right\rangle_{l}} \mathbf{u}_{l}^{j}(y) \\
& -\frac{\left\langle\cos \frac{(t+1) \pi}{h^{y}}\left(y-y_{l}\right), \mathbf{u}_{l}^{t+1}(y)\right\rangle_{l}}{\left\langle\mathbf{u}_{l}^{t+1}(y), \mathbf{u}_{l}^{t+1}(y)\right\rangle_{l}} \mathbf{u}_{l}^{t+1}(y) \sum_{i=0}^{s} \frac{\left\langle\cos \frac{(s+1) \pi}{h^{x}}\left(x-x_{k}\right), u_{k}^{i}(x)\right\rangle_{k}}{\left\langle u_{k}^{i}(x), u_{k}^{i}(x)\right\rangle_{k}} u_{k}^{i}(x)
\end{aligned}
$$




$$
\begin{aligned}
& -\sum_{i=0}^{s} \frac{\left\langle\cos \frac{(s+1) \pi}{h^{x}}\left(x-x_{k}\right), u_{k}^{i}(x)\right\rangle_{k}}{\left\langle u_{k}^{i}(x), u_{k}^{i}(x)\right\rangle_{k}} u_{k}^{i}(x) \sum_{j=0}^{t} \frac{\left\langle\cos \frac{(t+1) \pi}{h^{y}}\left(y-y_{l}\right), \mathbf{u}_{l}^{j}(y)\right\rangle_{l}}{\left\langle\mathbf{u}_{l}^{j}(y), \mathbf{u}_{l}^{j}(y)\right\rangle_{l}} \mathbf{u}_{l}^{j}(y) \\
= & \cos \frac{(s+1) \pi}{h^{x}}\left(x-x_{k}\right) \cos \frac{(t+1) \pi}{h^{y}}\left(y-y_{l}\right) \\
& -\frac{\left\langle\cos \frac{(s+1) \pi}{h^{x}}\left(x-x_{k}\right), \cos \frac{(s+1) \pi}{h^{x}}\left(x-x_{k}\right)-\cos \frac{s \pi}{h^{x}}\left(x-x_{k}\right)\right\rangle_{k}}{\left\langle u_{k}^{s+1}(x), u_{k}^{s+1}(x)\right\rangle_{k}} u_{k}^{s+1}(x) \frac{\left\langle\cos \frac{(t+1) \pi}{h^{y}}\left(y-y_{l}\right), \mathbf{u}_{l}^{t}(y)\right\rangle_{l}}{\left\langle\mathbf{u}_{l}^{t}(y), \mathbf{u}_{l}^{t}(y)\right\rangle_{l}} \mathbf{u}_{l}^{t}(y) \\
& -\frac{\left\langle\cos \frac{(t+1) \pi}{h^{y}}\left(y-y_{l}\right), \cos \frac{(t+1) \pi}{h^{y}}\left(y-y_{l}\right)-\cos \frac{t \pi}{h^{y}}\left(y-y_{l}\right)\right\rangle_{l}}{\left\langle\mathbf{u}_{l}^{t+1}(y), \mathbf{u}_{l}^{t+1}(y)\right\rangle_{l}} \mathbf{u}_{l}^{t+1}(y) \frac{\left\langle\cos \frac{(s+1) \pi}{h^{x}}\left(x-x_{k}\right), u_{k}^{s}(x)\right\rangle_{k}}{\left\langle u_{k}^{s}(x), u_{k}^{s}(x)\right\rangle_{k}} u_{k}^{s}(x) \\
& -\frac{\left\langle\cos \frac{(s+1) \pi}{h^{x}}\left(x-x_{k}\right), u_{k}^{s}(x)\right\rangle_{k}}{\left\langle u_{k}^{s}(x), u_{k}^{s}(x)\right\rangle_{k}} u_{k}^{s}(x) \frac{\left\langle\cos \frac{(t+1) \pi}{h^{y}}\left(y-y_{l}\right), \mathbf{u}_{l}^{t}(y)\right\rangle_{l}}{\left\langle\mathbf{u}_{l}^{t}(y), \mathbf{u}_{l}^{t}(y)\right\rangle_{l}} \mathbf{u}_{l}^{t}(y) \\
= & \cos \frac{(s+1) \pi}{h^{x}}\left(x-x_{k}\right) \cos \frac{(t+1) \pi}{h^{y}}\left(y-y_{l}\right)-u_{k}^{s+1}(x) \mathbf{u}_{l}^{t}(y)-u_{k}^{s}(x) \mathbf{u}_{l}^{t+1}(y)-u_{k}^{s}(x) \mathbf{u}_{l}^{t}(y) \\
= & \cos \frac{(s+1) \pi}{h^{x}}\left(x-x_{k}\right) \cos \frac{(t+1) \pi}{h^{y}}\left(y-y_{l}\right)-u_{k}^{s+1}(x) \mathbf{u}_{l}^{t}(y) \\
- & \left(\cos \frac{(t+1) \pi}{h^{y}}\left(y-y_{l}\right)-\mathbf{u}_{l}^{t}(y)\right) u_{k}^{s}(x)-u_{k}^{s}(x) \mathbf{u}_{l}^{t}(y) \\
= & \cos \frac{(t+1) \pi}{h^{y}}\left(y-y_{l}\right)\left(\cos \frac{(s+1) \pi}{h^{x}}\left(x-x_{k}\right)-u_{k}^{s}(x)\right)-u_{k}^{s+1}(x) \mathbf{u}_{l}^{t}(y) \\
= & \left(\cos \frac{(t+1) \pi}{h^{y}}\left(y-y_{l}\right)-\mathbf{u}_{l}^{t}(y)\right) u_{k}^{s+1}(x)=u_{k}^{s+1}(x) \mathbf{u}_{l}^{t+1}(y) .
\end{aligned}
$$

Case 2. Applying the Gram-Schmidt process to the system $\left\{\sin \frac{s \pi}{h^{x}}\left(x-x_{k}\right) \sin \frac{t \pi}{h^{y}}\left(y-y_{l}\right)\right\}$, we gain the orthogonal trigonometric functions $\mathcal{V}_{(k, l)}^{(s, t)}$ by the following recursive equations

$$
\mathcal{V}_{(k, l)}^{(1,1)}(x, y)=\sin \frac{\pi}{h^{x}}\left(x-x_{k}\right) \sin \frac{\pi}{h^{y}}\left(y-y_{l}\right)
$$

and for $s=1, \ldots, m, t=1, \ldots, n$

$$
\begin{aligned}
& \mathcal{V}_{(k, l)}^{(s, 1)}(x, y)=\sin \frac{s \pi}{h^{x}}\left(x-x_{k}\right) \sin \frac{\pi}{h^{y}}\left(y-y_{l}\right)-\sum_{i=1}^{s-1} \mu_{i, 1}^{(s, 1)} \mathcal{V}_{(k, l)}^{(i, 1)}(x, y), \\
& \mathcal{V}_{(k, l)}^{(1, t)}(x, y)=\sin \frac{t \pi}{h^{y}}\left(y-y_{l}\right) \sin \frac{\pi}{h^{x}}\left(x-x_{k}\right)-\sum_{j=1}^{t-1} \mu_{1, j}^{(1, t)} \mathcal{V}_{(k, l)}^{(1, j)}(x, y), \\
& \mathcal{V}_{(k, l)}^{(s, t)}(x, y)=\sin \frac{s \pi}{h^{x}}\left(x-x_{k}\right) \sin \frac{t \pi}{h^{y}}\left(y-y_{l}\right)-\sum_{j=1}^{t-1} \mu_{s, j}^{(s, t)} \mathcal{V}_{(k, l)}^{(s, j)}(x, y)-\sum_{i=1}^{s-1} \mu_{i, t}^{(s, t)} \mathcal{V}_{(k, l)}^{(i, t)}(x, y) \\
&-\sum_{i=1}^{s-1} \sum_{j=1}^{t-1} \mu_{i, j}^{(s, t)} \mathcal{V}_{(k, l)}^{(i, j)}(x, y),
\end{aligned}
$$

where

$$
\mu_{i, j}^{(s, t)}=\frac{\left\langle\sin \frac{s \pi}{h^{x}}\left(x-x_{k}\right) \sin \frac{t \pi}{h^{y}}\left(y-y_{l}\right), \mathcal{V}_{(k, l)}^{(i, j)}(x, y)\right\rangle_{k, l}}{\left\langle\mathcal{V}_{(k, l)}^{(i, j)}(x, y), \mathcal{V}_{(k, l)}^{(i, j)}(x, y)\right\rangle_{k, l}}, \quad i=1, \ldots, s, j=1, \ldots, t, \quad(\text { except } \mathrm{i}=\mathrm{s}, \mathrm{j}=\mathrm{t}) .
$$


We use the double mathematics induction in order to prove

$$
\mathcal{V}_{(k, l)}^{(s, t)}(x, y)=v_{k}^{s}(x) \mathbf{v}_{l}^{t}(y), \quad s=1, \ldots, m, t=1, \ldots, n .
$$

From Lemma 2.4 , it is clear that $\mathcal{V}_{(k, l)}^{(1,1)}(x, y)=v_{k}^{1}(x) \mathbf{v}_{l}^{1}(y)$. We consider the following mathematics induction hypothesis on $s$

$$
\mathcal{V}_{(k, l)}^{(s, 1)}(x, y)=v_{k}^{s}(x) \mathbf{v}_{l}^{1}(y)
$$

and we conclude

$$
\begin{aligned}
\mathcal{V}_{(k, l)}^{(s+1,1)}(x, y) & =\sin \frac{(s+1) \pi}{h^{x}}\left(x-x_{k}\right) \sin \frac{\pi}{h^{y}}\left(y-y_{l}\right)-\sum_{i=1}^{s} \mu_{i, 1}^{(s+1,1)} \mathcal{V}_{(k, l)}^{(i, 1)}(x, y) \\
& =\sin \frac{(s+1) \pi}{h^{x}}\left(x-x_{k}\right) \sin \frac{\pi}{h^{y}}\left(y-y_{l}\right) \\
& -\sum_{i=1}^{s} \frac{\left\langle\sin \frac{(s+1) \pi}{h^{x}}\left(x-x_{k}\right) \sin \frac{\pi}{h^{y}}\left(y-y_{l}\right), \mathcal{V}_{(k, l)}^{(i, 1)}(x, y)\right\rangle_{k, l}}{\left\langle\mathcal{V}_{(k, l)}^{(i, 1)}(x, y), \mathcal{V}_{(k, l)}^{(i, 1)}(x, y)\right\rangle_{k, l}} \mathcal{V}_{(k, l)}^{(i, 1)}(x, y) \\
& =\sin \frac{(s+1) \pi}{h^{x}}\left(x-x_{k}\right) \sin \frac{\pi}{h^{y}}\left(y-y_{l}\right)-\frac{s}{s+1} v_{k}^{s}(x) \mathbf{v}_{l}^{1}(y) \\
& =\mathbf{v}_{l}^{1}(y)\left(\sin \frac{(s+1) \pi}{h^{x}}\left(x-x_{k}\right)-\frac{s}{s+1} v_{k}^{s}(x)\right)=v_{k}^{s+1}(x) \mathbf{v}_{l}^{1}(y)
\end{aligned}
$$

In a similar way, we have $\mathcal{V}_{(k, l)}^{(1, t)}(x, y)=v_{k}^{1}(x) \mathbf{v}_{l}^{t}(y)$ for $t \in \mathbb{N}$.

Now, we assume $\mathcal{V}_{(k, l)}^{(s+1, t)}(x, y)=v_{k}^{s+1}(x) \mathbf{v}_{l}^{t}(y)$ and $\mathcal{V}_{(k, l)}^{(s, t+1)}(x, y)=v_{k}^{s}(x) \mathbf{v}_{l}^{t+1}(y)$ for some $s, t \in \mathbb{N}$, then we will conclude $\mathcal{V}_{(k, l)}^{(s+1, t+1)}(x, y)=v_{k}^{s+1}(x) \mathbf{v}_{l}^{t+1}(y)$. It follows from Eq. (4.2), that

$$
\begin{aligned}
\mathcal{V}_{(k, l)}^{(s+1, t+1)}(x, y) & =\sin \frac{(s+1) \pi}{h^{x}}\left(x-x_{k}\right) \sin \frac{(t+1) \pi}{h^{y}}\left(y-y_{l}\right) \\
& -\frac{\left\langle\sin \frac{(s+1) \pi}{h^{x}}\left(x-x_{k}\right), v_{k}^{s+1}(x)\right\rangle_{k}}{\left\langle v_{k}^{s+1}(x), v_{k}^{s+1}(x)\right\rangle_{k}} v_{k}^{s+1}(x) \sum_{j=1}^{t} \frac{\left\langle\sin \frac{(t+1) \pi}{h^{y}}\left(y-y_{l}\right), \mathbf{v}_{l}^{j}(y)\right\rangle_{l}}{\left\langle\mathbf{v}_{l}^{j}(y), \mathbf{v}_{l}^{j}(y)\right\rangle_{l}} \mathbf{v}_{l}^{j}(y) \\
& -\frac{\left\langle\sin \frac{(t+1) \pi}{h^{y}}\left(y-y_{l}\right), \mathbf{v}_{l}^{t+1}(y)\right\rangle_{l}}{\left\langle\mathbf{v}_{l}^{t+1}(y), \mathbf{v}_{l}^{t+1}(y)\right\rangle_{l}} \mathbf{v}_{l}^{t+1}(y) \sum_{i=1}^{s} \frac{\left\langle\sin \frac{(s+1) \pi}{h^{x}}\left(x-x_{k}\right), v_{k}^{i}(x)\right\rangle_{k}}{\left\langle v_{k}^{i}(x), v_{k}^{i}(x)\right\rangle_{k}} v_{k}^{i}(x)
\end{aligned}
$$




$$
\begin{aligned}
& -\sum_{i=1}^{s} \frac{\left\langle\sin \frac{(s+1) \pi}{h^{x}}\left(x-x_{k}\right), v_{k}^{i}(x)\right\rangle_{k}}{\left\langle v_{k}^{i}(x), v_{k}^{i}(x)\right\rangle_{k}} v_{k}^{i}(x) \sum_{j=1}^{t} \frac{\left\langle\sin \frac{(t+1) \pi}{h^{y}}\left(y-y_{l}\right), \mathbf{v}_{l}^{j}(y)\right\rangle_{l}}{\left\langle\mathbf{v}_{l}^{j}(y), \mathbf{v}_{l}^{j}(y)\right\rangle_{l}} \mathbf{v}_{l}^{j}(y) \\
= & \sin \frac{(s+1) \pi}{h^{x}}\left(x-x_{k}\right) \sin \frac{(t+1) \pi}{h^{y}}\left(y-y_{l}\right) \\
& -\frac{\left\langle\sin \frac{(s+1) \pi}{h^{x}}\left(x-x_{k}\right), \sin \frac{(s+1) \pi}{h^{x}}\left(x-x_{k}\right)-\frac{s}{s+1} \sin \frac{s \pi}{h^{x}}\left(x-x_{k}\right)\right\rangle_{k}}{\left\langle v_{k}^{s+1}(x), v_{k}^{s+1}(x)\right\rangle_{k}} v_{k}^{s+1}(x) \\
& \frac{\left\langle\sin \frac{(t+1) \pi}{h^{y}}\left(y-y_{l}\right), \sin \frac{t \pi}{h^{y}}\left(y-y_{l}\right)\right\rangle_{l}}{\left\langle\mathbf{v}_{l}^{t}(y), \mathbf{v}_{l}^{t}(y)\right\rangle_{l}}(y) \\
& -\frac{\left\langle\sin \frac{(t+1) \pi}{h^{y}}\left(y-y_{l}\right), \sin \frac{(t+1) \pi}{h^{y}}\left(y-y_{l}\right)-\frac{t}{t+1} \sin \frac{t \pi}{h^{y}}\left(y-y_{l}\right)\right\rangle_{l}}{\left\langle\mathbf{v}_{l}^{t+1}(y), \mathbf{v}_{l}^{t+1}(y)\right\rangle_{l}} \\
& \frac{\left\langle\sin \frac{(s+1) \pi}{h^{x}}\left(x-x_{k}\right), \sin \frac{s \pi}{h^{x}}\left(x-x_{k}\right)\right\rangle_{k}}{\left\langle v_{k}^{s}(x), v_{k}^{s}(x)\right\rangle_{k}} v_{k}^{s}(x) \\
- & \frac{\left\langle\sin \frac{(s+1) \pi}{h^{x}}\left(x-x_{k}\right), \sin \frac{s \pi}{h^{x}}\left(x-x_{k}\right)\right\rangle_{k}}{\left\langle v_{k}^{s}(x), v_{k}^{s}(x)\right\rangle_{k}} v_{k}^{s}(x) \frac{\left\langle\sin \frac{(t+1) \pi}{h^{y}}\left(y-y_{l}\right), \sin \frac{t \pi}{h^{y}}\left(y-y_{l}\right)\right\rangle_{l}}{\left\langle\mathbf{v}_{l}^{t}(y), \mathbf{v}_{l}^{t}(y)\right\rangle_{l}} \mathbf{v}_{l}^{t}(y) \\
= & \sin \frac{(s+1) \pi}{h^{x}}\left(x-x_{k}\right) \sin \frac{(t+1) \pi}{h^{y}}\left(y-y_{l}\right) \\
- & \frac{t}{t+1}\left(s i n \frac{(s+1) \pi}{h^{x}}\left(x-x_{k}\right)-\frac{s}{s+1} v_{k}^{s}(x)\right) \mathbf{v}_{l}^{t}(y)-\frac{s}{s+1} \mathbf{v}_{l}^{t+1}(y) v_{k}^{s}(x) \\
- & \left(\frac{s}{s+1}\right)\left(\frac{t}{t+1}\right) \mathbf{v}_{l}^{t}(y) v_{k}^{s}(x)=\sin \frac{(s+1) \pi}{h^{x}}\left(x-x_{k}\right)\left(\sin \frac{(t+1) \pi}{h^{y}}\left(y-y_{l}\right)-\frac{t}{t+1} \mathbf{v}_{l}^{t}(y)\right) \\
- & \left(\frac{s}{s+1}\right) \mathbf{v}_{l}^{t+1}(y) v_{k}^{s}(x)=v_{k}^{s+1}(x) \mathbf{v}_{l}^{t+1}(y) .
\end{aligned}
$$

The proof of Cases 3 and 4 are similar.

Lemma 4.2. The system $\left\{\mathcal{U}_{(k, l)}^{(s, t)}(x, y), \mathcal{V}_{(k, l)}^{(s, t)}(x, y), \mathcal{X}_{(k, l)}^{(s, t)}(x, y), \mathcal{Y}_{(k, l)}^{(s, t)}(x, y)\right\}$ is orthogonal and linearly independent in $L_{2}\left(\bar{A}_{k, l}\right)$.

Proof. From Lemmas 2.4, 4.1 and properties of inner product, we have

$$
\left\langle\mathcal{U}_{(k, l)}^{(s, t)}(x, y), \mathcal{V}_{(k, l)}^{(s, t)}(x, y)\right\rangle_{k, l}=\left\langle u_{k}^{s}(x), v_{k}^{s}(x)\right\rangle_{k}\left\langle\mathbf{u}_{l}^{t}(y), \mathbf{v}_{l}^{t}(y)\right\rangle_{l}=0 .
$$

In a similar way as above, we can immediately conclude that the set

$$
\left\{\mathcal{U}_{(k, l)}^{(s, t)}(x, y), \mathcal{V}_{(k, l)}^{(s, t)}(x, y), \mathcal{X}_{(k, l)}^{(s, t)}(x, y), \mathcal{Y}_{(k, l)}^{(s, t)}(x, y)\right\},
$$

is orthogonal in $L_{2}\left(\bar{A}_{k, l}\right)$. In order to proof linear independence of the mentioned systems, we consider

$$
a \mathcal{U}_{(k, l)}^{(s, t)}(x, y)+b \mathcal{V}_{(k, l)}^{(s, t)}(x, y)+c \mathcal{X}_{(k, l)}^{(s, t)}(x, y)+d \mathcal{Y}_{(k, l)}^{(s, t)}(x, y)=0 .
$$

From definition of inner product, we have

$$
\left\langle a \mathcal{U}_{(k, l)}^{(s, t)}(x, y)+b \mathcal{V}_{(k, l)}^{(s, t)}(x, y)+c \mathcal{X}_{(k, l)}^{(s, t)}(x, y)+d \mathcal{Y}_{(k, l)}^{(s, t)}(x, y), \mathcal{U}_{(k, l)}^{(s, t)}(x, y)\right\rangle_{k, l}=0 .
$$

From the orthogonality of mentioned set, we conclude that

$$
a\left\langle\mathcal{U}_{(k, l)}^{(s, t)}(x, y), \mathcal{U}_{(k, l)}^{(s, t)}(x, y)\right\rangle_{k, l}=0 .
$$

Therefore $a=0$. Similarly, we can conclude $b=c=d=0$.

It shows that the set $\left\{\mathcal{U}_{(k, l)}^{(s, t)}(x, y), \mathcal{V}_{(k, l)}^{(s, t)}(x, y), \mathcal{X}_{(k, l)}^{(s, t)}(x, y), \mathcal{Y}_{(k, l)}^{(s, t)}(x, y)\right\}$ is linearly independent. 
Remark 4.3. Let $m, n \geq 0$. We denote by $L_{2}^{m n}\left(\bar{A}_{k, l}\right)$, the linear subspace of $L_{2}\left(\bar{A}_{k, l}\right)$ spanned by trigonometric basis $\left\{\mathcal{U}_{(k, l)}^{(s, t)}, \mathcal{V}_{(k, l)}^{(s, t)}, \mathcal{X}_{(k, l)}^{(s, t)}, \mathcal{Y}_{(k, l)}^{(s, t)}\right\}$.

Lemma 4.4. Let trigonometric functions ${ }^{t} \bar{F}_{k l}^{m n}$ be the orthogonal projection of $f \in L_{2}\left(\bar{A}_{k, l}\right)$ on $L_{2}^{m n}\left(\bar{A}_{k, l}\right)$. Then,

$$
\begin{aligned}
{ }^{t} \bar{F}_{k l}^{m n}(x, y) & =\sum_{s=0}^{m} \sum_{t=0}^{n} a_{(k, l)}^{(s, t)} \mathcal{U}_{(k, l)}^{(s, t)}(x, y)+\sum_{s=1}^{m} \sum_{t=1}^{n} b_{(k, l)}^{(s, t)} \mathcal{V}_{(k, l)}^{(s, t)}(x, y) \\
& +\sum_{s=0}^{m} \sum_{t=1}^{n} c_{(k, l)}^{(s, t)} \mathcal{X}_{(k, l)}^{(s, t)}(x, y)+\sum_{s=1}^{m} \sum_{t=0}^{n} d_{(k, l)}^{(s, t)} \mathcal{Y}_{(k, l)}^{(s, t)}(x, y)
\end{aligned}
$$

where

$a_{(k, l)}^{(0,0)}=\frac{1}{4 h^{x} h^{y}} \int_{y_{l-1}}^{y_{l+1}} \int_{x_{k-1}}^{x_{k+1}} \bar{f}(x, y)\left(\cos \frac{\pi}{h^{x}}\left(x-x_{k}\right)+1\right)\left(\cos \frac{\pi}{h^{y}}\left(y-y_{l}\right)+1\right) d x d y$,

for $t=1, \ldots, n$

$a_{(k, l)}^{(0, t)}=\frac{1}{2 h^{x} h^{y}} \int_{y_{l-1}}^{y_{l+1}} \int_{x_{k-1}}^{x_{k+1}} \bar{f}(x, y)\left(\cos \frac{(t+1) \pi}{h^{y}}\left(y-y_{l}\right)+\cos \frac{t \pi}{h^{y}}\left(y-y_{l}\right)\right)\left(\cos \frac{\pi}{h^{x}}\left(x-x_{k}\right)+1\right) d x d y$,

for $s=1, \ldots, m$

$a_{(k, l)}^{(s, 0)}=\frac{1}{2 h^{x} h^{y}} \int_{y_{l-1}}^{y_{l+1}} \int_{x_{k-1}}^{x_{k+1}} \bar{f}(x, y)\left(\cos \frac{(s+1) \pi}{h^{x}}\left(x-x_{k}\right)+\cos \frac{s \pi}{h^{x}}\left(x-x_{k}\right)\right)\left(\cos \frac{\pi}{h^{y}}\left(y-y_{l}\right)+1\right) d x d y$,

for $s=1, \ldots, m, t=1, \ldots, n$

$a_{(k, l)}^{(s, t)}=\frac{1}{h^{x} h^{y}} \int_{y_{l-1}}^{y_{l+1}} \int_{x_{k-1}}^{x_{k+1}} \bar{f}(x, y)\left(\cos \frac{(s+1) \pi}{h^{x}}\left(x-x_{k}\right)+\cos \frac{s \pi}{h^{x}}\left(x-x_{k}\right)\right)$

$$
\left(\cos \frac{(t+1) \pi}{h^{y}}\left(y-y_{l}\right)+\cos \frac{t \pi}{h^{y}}\left(y-y_{l}\right)\right) d x d y,
$$

$b_{(k, l)}^{(s, t)}=\frac{1}{h^{x} h^{y}} \int_{y_{l-1}}^{y_{l+1}} \int_{x_{k-1}}^{x_{k+1}} \bar{f}(x, y)\left(\frac{s}{s+1} \sin \frac{(s+1) \pi}{h^{x}}\left(x-x_{k}\right)+\sin \frac{s \pi}{h^{x}}\left(x-x_{k}\right)\right)$

$$
\left(\frac{t}{t+1} \sin \frac{(t+1) \pi}{h^{y}}\left(y-y_{l}\right)+\sin \frac{t \pi}{h^{y}}\left(y-y_{l}\right)\right) d x d y,
$$

for $t=1, \ldots, n$

$c_{(k, l)}^{(0, t)}=\frac{1}{2 h^{x} h^{y}} \int_{y_{l-1}}^{y_{l+1}} \int_{x_{k-1}}^{x_{k+1}} \bar{f}(x, y)\left(\frac{t}{t+1} \sin \frac{(t+1) \pi}{h^{y}}\left(y-y_{l}\right)+\sin \frac{t \pi}{h^{y}}\left(y-y_{l}\right)\right)\left(\cos \frac{\pi}{h^{x}}\left(x-x_{k}\right)+1\right) d x d y$,

for $s=1, \ldots, m, t=1, \ldots, n$

$c_{(k, l)}^{(s, t)}=\frac{1}{h^{x} h^{y}} \int_{y_{l-1}}^{y_{l+1}} \int_{x_{k-1}}^{x_{k+1}} \bar{f}(x, y)\left(\frac{t}{t+1} \sin \frac{(t+1) \pi}{h^{y}}\left(y-y_{l}\right)+\sin \frac{t \pi}{h^{y}}\left(y-y_{l}\right)\right)$

$$
\left(\cos \frac{(s+1) \pi}{h^{x}}\left(x-x_{k}\right)+\cos \frac{s \pi}{h^{x}}\left(x-x_{k}\right)\right) d x d y,
$$

for $s=1, \ldots, m$

$d_{(k, l)}^{(s, 0)}=\frac{1}{2 h^{x} h^{y}} \int_{y_{l-1}}^{y_{l+1}} \int_{x_{k-1}}^{x_{k+1}} \bar{f}(x, y)\left(\frac{s}{s+1} \sin \frac{(s+1) \pi}{h^{x}}\left(x-x_{k}\right)+\sin \frac{s \pi}{h^{x}}\left(x-x_{k}\right)\right)\left(\cos \frac{\pi}{h^{y}}\left(y-y_{l}\right)+1\right) d x d y$, 
and for $s=1, \ldots, m, t=1, \ldots, n$

$$
\begin{aligned}
& d_{(k, l)}^{(s, t)}=\frac{1}{h^{x} h^{y}} \int_{y_{l-1}}^{y_{l+1}} \int_{x_{k-1}}^{x_{k+1}} \bar{f}(x, y)\left(\frac{s}{s+1} \sin \frac{(s+1) \pi}{h^{x}}\left(x-x_{k}\right)+\sin \frac{s \pi}{h^{x}}\left(x-x_{k}\right)\right) \\
& \left(\cos \frac{(t+1) \pi}{h^{y}}\left(y-y_{l}\right)+\cos \frac{t \pi}{h^{y}}\left(y-y_{l}\right)\right) d x d y .
\end{aligned}
$$

Proof. It follows from the assumption of the present theorem that $\bar{f}-{ }^{t} \bar{F}_{k l}^{m n} \perp L_{2}^{m n}\left(\bar{A}_{k, l}\right)$. Utilizing the properties of orthogonality along with the basic functions of $L_{2}^{m n}\left(\bar{A}_{k, l}\right)$, we arrive at

$a_{(k, l)}^{(s, t)}=\frac{\left\langle\bar{f}, \mathcal{U}_{(k, l)}^{(s, t)}\right\rangle_{k, l}}{\left\langle\mathcal{U}_{(k, l)}^{(s, t)}, \mathcal{U}_{(k, l)}^{s, t)}\right\rangle_{k, l}}, \quad b_{(k, l)}^{(s, t)}=\frac{\left\langle\bar{f}, \mathcal{V}_{(k, l)}^{(s, t)}\right\rangle_{k, l}}{\left\langle\mathcal{V}_{(k, l)}^{(s, t)}, \mathcal{V}_{(k, l)}^{(s, t)}\right\rangle_{k, l}}, \quad c_{(k, l)}^{(s, t)}=\frac{\left\langle\bar{f}, \mathcal{X}_{(k, l)}^{(s, t)}\right\rangle_{k, l}}{\left\langle\mathcal{X}_{(k, l)}^{(s, t)}, \mathcal{X}_{(k, l)}^{(s, t)}\right\rangle_{k, l}}, \quad d_{(k, l)}^{(s, t)}=\frac{\left\langle\bar{f}, \mathcal{Y}_{(k, l)}^{(s, t)}\right\rangle_{k, l}}{\left\langle\mathcal{Y}_{(k, l)}^{(s, t)}, \mathcal{Y}_{(k, l)}^{(s, t)}\right\rangle_{k, l}}$

From Lemmas 2.4, 4.1 and definition of inner product, we can immediately complete the proof.

\subsection{Direct and inverse trigonometric $\bar{F}^{m n}$-transforms}

Here, we generalize the direct and inverse ${ }^{t} \bar{F}^{m}$-transform of one-variable functions to two-variable functions and we call it ${ }^{t} \bar{F}^{m n}$-transform. This is also a generalization of the $\bar{F}$-transform of two-variable functions.

Definition 4.5. Let $\bar{A}_{k, l}$ be the extended sinusoidal shaped basic functions and $\left\{\mathcal{U}_{(k, l)}^{(s, t)}, \mathcal{V}_{(k, l)}^{(s, t)}, \mathcal{X}_{(k, l)}^{(s, t)}, \mathcal{Y}_{(k, l)}^{(s, t)}\right\}$ be the trigonometric basis of $L_{2}^{m n}\left(\bar{A}_{k, l}\right)$. A direct ${ }^{t} \bar{F}^{m n}$-transform of a function $f \in L_{2}\left(\bar{A}_{k, l}\right)$ is defined as a matrix ${ }^{t} \bar{F}^{m n}(f)=\left[{ }^{t} \bar{F}_{k l}^{m n}\right]_{p \times q}$ such that $k l$-th component ${ }^{t} \bar{F}_{k l}^{m n}$ is given as follows

${ }^{t} \bar{F}_{k l}^{m n}(x, y)=\sum_{s=0}^{m} \sum_{t=0}^{n} a_{(k, l)}^{(s, t)} \mathcal{U}_{(k, l)}^{(s, t)}(x, y)+\sum_{s=1}^{m} \sum_{t=1}^{n} b_{(k, l)}^{(s, t)} \mathcal{V}_{(k, l)}^{(s, t)}(x, y)+\sum_{s=0}^{m} \sum_{t=1}^{n} c_{(k, l)}^{(s, t)} \mathcal{X}_{(k, l)}^{(s, t)}(x, y)+\sum_{s=1}^{m} \sum_{t=0}^{n} d_{(k, l)}^{(s, t)} \mathcal{Y}_{(k, l)}^{(s, t)}(x, y)$,

where $a_{(k, l)}^{(s, t)}, b_{(k, l)}^{(s, t)}, c_{(k, l)}^{(s, t)}$, and $d_{(k, l)}^{(s, t)}$ have been defined in Lemma 4.4.

Lemma 4.6. The ${ }^{t} \bar{F}^{m n}$-transform is a generalization of the $\bar{F}$-transform of two-variable functions.

Proof. Let $m, n=0$, and $\left[{ }^{t} \bar{F}_{k l}^{00}\right]_{p \times q}$ be the ${ }^{t} \bar{F}^{m n}$-transform of $f$. We claim that

$$
\left[{ }^{t} \bar{F}_{k l}^{00}\right]_{p \times q}=\left[\bar{F}_{k l}\right]_{p \times q}
$$

We know that ${ }^{t} \bar{F}_{k l}^{00}=a_{(k, l)}^{(0,0)}$ for $k=1, \cdots, p$ and $l=1, \cdots, q$. On the other hand, we have

$$
a_{(k, l)}^{(0,0)}=\frac{\left\langle\bar{f}, \mathcal{U}_{(k, l)}^{(0,0)}\right\rangle_{k, l}}{\left\langle\mathcal{U}_{(k, l)}^{(0,0)}, \mathcal{U}_{(k, l)}^{(0,0)}\right\rangle_{k, l}}=\frac{\int_{y_{l-1}}^{y_{l+1}} \int_{x_{k-1}}^{x_{k+1}} \bar{f}(x, y) \bar{A}_{k, l}(x, y) d x d y}{\int_{y_{l-1}}^{y_{l+1}} \int_{x_{k-1}}^{x_{k+1}} \bar{A}_{k, l}(x, y) d x d y}=\bar{F}_{k l} \quad k=1, \ldots, p, l=1, \ldots, q .
$$

This completes the proof.

Definition 4.7. Let $\bar{A}_{k, l}$ be the extended sinusoidal shaped basic functions. Let $f:[a, b] \times[c, d] \rightarrow \mathbb{R}$ be a given function, $(m, n \geq 0)$ and let $\left[{ }^{t} \bar{F}_{k l}^{m n}\right]_{p \times q}$ be the ${ }^{t} \bar{F}^{m n}$-transform of $f$. Then, the following function ${ }^{t} \stackrel{\hat{\hat{f}}}{p q}^{m n}:\left[a-h^{x}, b+h^{x}\right] \times\left[c-h^{y}, d+h^{y}\right] \rightarrow \mathbb{R}$

$$
{ }^{t} \overline{\hat{f}}_{p q}^{m n}(f(x, y))=\sum_{k=1}^{p} \sum_{l=1}^{q}{ }^{t} \bar{F}_{k l}^{m n} \bar{A}_{k, l}(x, y),
$$

is called the inverse ${ }^{t} \bar{F}^{m n}$-transform of $f$. 


\section{Error analysis of the direct and inverse ${ }^{t} \bar{F}^{m n}$-transform}

In this section, using the trapezium formula (Euler-Maclaurin Summation Formula in [31]) for approximating integrals, the error analysis of the direct and inverse ${ }^{t} \bar{F}^{m n}$-transform of a given function is investigated.

\subsection{Error analysis of the direct ${ }^{t} \bar{F}^{m n}$-transform}

Theorem 5.1. Let $f \in L_{2}\left(\bar{A}_{k, l}\right)$ and $\bar{f}$ be four times continuously differentiable w.r.t. $x$ and $y$ in $\left[a-h^{x}, b+h^{x}\right] \times\left[c-h^{y}, d+h^{y}\right]$. Moreover, let ${ }^{t} \bar{F}_{k l}^{m n}$ be the ${ }^{t} \bar{F}^{m n}$-transform components of $f$. Then for every $k=1, \ldots, p, l=1, \ldots, q$

$$
{ }^{t} \bar{F}_{k l}^{m n}(x, y)=\bar{f}(x, y)+O\left(\max \left(\left(h^{x}\right)^{3},\left(h^{y}\right)^{3}\right)\right), \quad(x, y) \in\left[x_{k-1}, x_{k+1}\right] \times\left[y_{l-1}, y_{l+1}\right] .
$$

Proof. We are going to approximate integrals (4.3)-(4.11) with the help of the trapezium formula as follows

$$
\begin{aligned}
a_{(k, l)}^{(0,0)} & =\bar{f}\left(x_{k}, y_{l}\right)+O\left(\max \left(\left(h^{x}\right)^{3},\left(h^{y}\right)^{3}\right)\right), & & a_{(k, l)}^{(s, 0)}=O\left(\max \left(\left(h^{x}\right)^{3},\left(h^{y}\right)^{3}\right)\right) \\
a_{(k, l)}^{(0, t)} & =O\left(\max \left(\left(h^{x}\right)^{3},\left(h^{y}\right)^{3}\right)\right), & & a_{(k, l)}^{(s, t)}=O\left(\max \left(\left(h^{x}\right)^{3},\left(h^{y}\right)^{3}\right)\right) \\
b_{(k, l)}^{(s, t)} & =O\left(\max \left(\left(h^{x}\right)^{3},\left(h^{y}\right)^{3}\right)\right), & & c_{(k, l)}^{(0, t)}=O\left(\max \left(\left(h^{x}\right)^{3},\left(h^{y}\right)^{3}\right)\right) \\
c_{(k, l)}^{(s, t)} & =O\left(\max \left(\left(h^{x}\right)^{3},\left(h^{y}\right)^{3}\right)\right), & & d_{(k, l)}^{(s, 0)}=O\left(\max \left(\left(h^{x}\right)^{3},\left(h^{y}\right)^{3}\right)\right) \\
d_{(k, l)}^{(s, t)} & =O\left(\max \left(\left(h^{x}\right)^{3},\left(h^{y}\right)^{3}\right)\right) . & &
\end{aligned}
$$

We just approximate Eq. (4.8) and the others are approximated similarly. We assume

$$
I(x)=\int_{y_{l-1}}^{y_{l+1}} \bar{f}(x, y)\left(\frac{t}{t+1} \sin \frac{(t+1) \pi}{h^{y}}\left(y-y_{l}\right)+\sin \frac{t \pi}{h^{y}}\left(y-y_{l}\right)\right) d y .
$$

It follows from Eq. (4.8), that

$$
c_{(k, l)}^{(0, t)}=\frac{1}{2 h^{x} h^{y}} \int_{x_{k-1}}^{x_{k+1}} I(x)\left(\cos \frac{\pi}{h^{x}}\left(x-x_{k}\right)+1\right) d x .
$$

Five nodes $\left\{y_{l-1}, y_{l}-\frac{h^{y}}{2}, y_{l}, y_{l}+\frac{h^{y}}{2}, y_{l+1}\right\}$ are considered in the trapezium formula in order to approximate $I(x)$.

$$
I(x)=\frac{h^{y}}{2}\left(\frac{t}{t+1} \sin \frac{(t+1) \pi}{2}+\sin \frac{t \pi}{2}\right)\left(\bar{f}\left(x, y_{l}+\frac{h^{y}}{2}\right)-\bar{f}\left(x, y_{l}-\frac{h^{y}}{2}\right)\right)+O\left(\left(h^{y}\right)^{4}\right) .
$$

On the other hand, the term $\bar{f}\left(x, y_{l}+\frac{h^{y}}{2}\right)-\bar{f}\left(x, y_{l}-\frac{h^{y}}{2}\right)$ can be estimated by Taylor expansion as follows

$$
\bar{f}\left(x, y_{l}+\frac{h^{y}}{2}\right)-\bar{f}\left(x, y_{l}-\frac{h^{y}}{2}\right)=h^{y} \frac{\partial}{\partial y} \bar{f}\left(x, y_{l}\right)+O\left(\left(h^{y}\right)^{3}\right) .
$$

Putting Eq. (5.3) into (5.2), we conclude that

$$
I(x)=M_{t} h^{y}\left(h^{y} \frac{\partial}{\partial y} \bar{f}\left(x, y_{l}\right)+O\left(\left(h^{y}\right)^{3}\right)\right)+O\left(\left(h^{y}\right)^{3}\right),
$$

where $M_{t}=\frac{t}{2 t+2} \sin \frac{(t+1) \pi}{2}+\frac{1}{2} \sin \frac{t \pi}{2}$. Similarly, we approximate Eq. (5.1) with the help of the trapezium formula with three nodes $\left\{x_{k-1}, x_{k}, x_{k+1}\right\}$ as follows

$$
c_{(k, l)}^{(0, t)}=M_{t} h^{y} \frac{\partial \bar{f}}{\partial y}\left(x_{k}, y_{l}\right)+O\left(\max \left(\left(h^{x}\right)^{3},\left(h^{y}\right)^{3}\right)\right) .
$$


By Definition 4.5 and the above approximations, we deduce that

$$
\begin{aligned}
& { }^{t} \bar{F}_{k l}^{m n}(x, y)=a_{(k, l)}^{(0,0)} \mathcal{U}_{(k, l)}^{(0,0)}(x, y)+\sum_{t=1}^{n} a_{(k, l)}^{(0, t)} \mathcal{U}_{(k, l)}^{(0, t)}(x, y)+\sum_{s=1}^{m} a_{(k, l)}^{(s, 0)} \mathcal{U}_{(k, l)}^{(s, 0)}(x, y)+\sum_{s=1}^{m} \sum_{t=1}^{n} a_{(k, l)}^{(s, t)} \mathcal{U}_{(k, l)}^{(s, t)}(x, y) \\
& +\sum_{s=1}^{m} \sum_{t=1}^{n} b_{(k, l)}^{(s, t)} \mathcal{V}_{(k, l)}^{(s, t)}(x, y)+\sum_{t=1}^{n} c_{(k, l)}^{(0, t)} \mathcal{X}_{(k, l)}^{(0, t)}(x, y)+\sum_{s=1}^{m} \sum_{t=1}^{n} c_{(k, l)}^{(s, t)} \mathcal{X}_{(k, l)}^{(s, t)}(x, y)+\sum_{s=1}^{m} d_{(k, l)}^{(s, 0)} \mathcal{Y}_{(k, l)}^{(s, 0)}(x, y) \\
& +\sum_{s=1}^{m} \sum_{t=1}^{n} d_{(k, l)}^{(s, t)} \mathcal{Y}_{(k, l)}^{(s, t)}(x, y) \\
& =\bar{f}\left(x_{k}, y_{l}\right)+O\left(\max \left(\left(h^{x}\right)^{3},\left(h^{y}\right)^{3}\right)\right)+O\left(\max \left(\left(h^{x}\right)^{3},\left(h^{y}\right)^{3}\right)\right)\left(\sum_{t=1}^{n} \sum_{j=1}^{t}(-1)^{t+j} \cos \frac{j \pi}{h^{y}}\left(y-y_{l}\right)+(-1)^{t} \frac{1}{2}\right. \\
& +\sum_{s=1}^{m} \sum_{i=1}^{s}(-1)^{s+i} \cos \frac{i \pi}{h^{x}}\left(x-x_{k}\right)+(-1)^{s} \frac{1}{2} \\
& \left.+\sum_{s=1}^{m} \sum_{t=1}^{n} \sum_{i=1}^{s} \sum_{j=1}^{t}\left((-1)^{s+i} \cos \frac{i \pi}{h^{x}}\left(x-x_{k}\right)+(-1)^{s} \frac{1}{2}\right)\left((-1)^{t+j} \cos \frac{j \pi}{h^{y}}\left(y-y_{l}\right)+(-1)^{t} \frac{1}{2}\right)\right) \\
& +O\left(\max \left(\left(h^{x}\right)^{3},\left(h^{y}\right)^{3}\right)\right) \sum_{s=1}^{m} \sum_{t=1}^{n} \sum_{i=1}^{s} \sum_{j=1}^{t}\left((-1)^{s+i} \frac{i}{s} \sin \frac{i \pi}{h^{x}}\left(x-x_{k}\right)\right)\left((-1)^{t+j} \frac{j}{t} \sin \frac{j \pi}{h^{y}}\left(y-y_{l}\right)\right) \\
& \left.+O\left(\max \left(\left(h^{x}\right)^{3},\left(h^{y}\right)^{3}\right)\right)\left(\sum_{t=1}^{q} \sum_{j=1}^{t}(-1)^{t+j} \frac{j}{t} \sin \frac{j \pi}{h^{y}}\left(y-y_{l}\right)+\sum_{s=1}^{m} \sum_{i=1}^{s}(-1)^{s+i} \frac{i}{s} \sin \frac{i \pi}{h^{x}}\left(x-x_{k}\right)\right)\right) \\
& +O\left(\max \left(\left(h^{x}\right)^{3},\left(h^{y}\right)^{3}\right)\right)\left(\sum_{s=1}^{m} \sum_{t=1}^{n} \sum_{i=1}^{s} \sum_{j=1}^{t}\left((-1)^{s+i} \cos \frac{i \pi}{h^{x}}\left(x-x_{k}\right)+(-1)^{s} \frac{1}{2}\right)\left((-1)^{t+j} \frac{j}{t} \sin \frac{j \pi}{h^{y}}\left(y-y_{l}\right)\right)\right. \\
& \left.+\sum_{s=1}^{m} \sum_{t=1}^{n} \sum_{i=1}^{s} \sum_{j=1}^{t}\left((-1)^{s+i} \frac{i}{s} \sin \frac{i \pi}{h^{x}}\left(x-x_{k}\right)\right)\left((-1)^{t+j} \cos \frac{j \pi}{h^{y}}\left(y-y_{l}\right)+(-1)^{t} \frac{1}{2}\right)\right) .
\end{aligned}
$$

Applying Taylor expansion for sin and cos appeared above, we conclude

$$
{ }^{t} \bar{F}_{k l}^{m n}(x, y)=\bar{f}\left(x_{k}, y_{l}\right)+O\left(\max \left(\left(h^{x}\right)^{3},\left(h^{y}\right)^{3}\right)\right)=\bar{f}(x, y)+O\left(\max \left(\left(h^{x}\right)^{3},\left(h^{y}\right)^{3}\right)\right) .
$$

Remark 5.2. It worth noting that if $(x, y) \in\left[x_{k-1}, x_{k+1}\right] \times\left[y_{l-1}, y_{l+1}\right]$ for $k=2, \ldots, p-1, l=2, \ldots, q-1$, then

$$
{ }^{t} \bar{F}_{k l}^{m n}(x, y)=f(x, y)+O\left(\max \left(\left(h^{x}\right)^{3},\left(h^{y}\right)^{3}\right)\right) .
$$

The following lemma shows that if we increase $m$ and $n$, then the approximation property of the components of the direct ${ }^{t} \bar{F}^{m n}$-transform get better.

Lemma 5.3. Let $\bar{A}_{k, l}$ be sinusoidal shaped basic functions. Moreover, let trigonometric functions ${ }^{t} \bar{F}_{k l}^{m n}$, ${ }^{t} \bar{F}_{k l}^{(m+1)(n+1)}(m, n \geq 0)$ be orthogonal projections of $f \in L_{2}\left(\bar{A}_{k, l}\right)$ on $L_{2}^{m n}\left(\bar{A}_{k, l}\right)$ and $L_{2}^{(m+1)(n+1)}\left(\bar{A}_{k, l}\right)$, respectively. Then,

$$
\left\|\left.f\right|_{\left[x_{k-1}, x_{k+1}\right] \times\left[y_{l-1}, y_{l+1}\right]}-{ }^{t} \bar{F}_{k l}^{(m+1)(n+1)}\right\|_{k l} \leq\left\|\left.f\right|_{\left[x_{k-1}, x_{k+1}\right] \times\left[y_{l-1}, y_{l+1}\right]}-{ }^{t} \bar{F}_{k l}^{m n}\right\|_{k l} .
$$

Proof. Our proof is similar to Lemma 2 in [32].

\subsection{Error analysis of the inverse ${ }^{t} \bar{F}^{m n}$-transform}

In this section, we give an error estimation of the inverse ${ }^{t} \bar{F}^{m n}$-transform in the space of continuous functions. 
Theorem 5.4. Let $\bar{f}$ be four times continuously differentiable on $\left[a-h^{x}, b+h^{x}\right] \times\left[c-h^{y}, d+h^{y}\right]$. Let $\bar{A}_{k, l}$ be sinusoidal shaped functions and ${ }^{t} \overline{\hat{f}}_{p q}^{m n}$ be the inverse ${ }^{t} \bar{F}^{m n}$-transform of $f$. Then, for $(x, y) \in[a, b] \times[c, d]$, the following approximation is derived

$$
f(x, y)-{ }^{t} \overline{\hat{f}}_{p q}^{m n}(f(x, y))=O\left(\max \left(\left(h^{x}\right)^{3},\left(h^{y}\right)^{3}\right)\right) .
$$

Proof. Utilizing the proof of Theorem 3 in [8] for $(x, y) \in[a, b] \times[c, d]$, we have $\sum_{k=1}^{p} A_{k}(x)=\sum_{l=1}^{q} A_{l}(y)=$ 1. Therefore, we conclude

$$
\sum_{k=1}^{p} \sum_{l=1}^{q} \bar{A}_{k, l}(x, y)=\sum_{k=1}^{p} \sum_{l=1}^{q} A_{k, l}(x, y)=\sum_{k=1}^{p} A_{k}(x) \sum_{l=1}^{q} A_{l}(y)=1 .
$$

From Remark 5.2, we deduce that there are $k \in\{1, \cdots, p-1\}$ and $l \in\{1, \cdots, q-1\}$ such that $(x, y) \in\left[x_{k}, x_{k+1}\right] \times\left[y_{l}, y_{l+1}\right]$. Therefore, we have

$$
\begin{aligned}
& f(x, y)-{ }^{t} \overline{\hat{f}}_{p q}^{m n}(f(x, y))=f(x, y)-\sum_{k=1}^{p} \sum_{l=1}^{q}{ }^{t} \bar{F}_{k l}^{m n}(x, y) A_{k, l}(x, y)=f(x, y) \sum_{k=1}^{p} \sum_{l=1}^{q} A_{k, l}(x, y) \\
& -\sum_{k=1}^{p} \sum_{l=1}^{q}{ }^{t} \bar{F}_{k l}^{m n}(x, y) A_{k, l}(x, y)=\sum_{k=1}^{p} \sum_{l=1}^{q} A_{k, l}(x, y)\left(f(x, y)-{ }^{t} \bar{F}_{k l}^{m n}(x, y)\right)=O\left(\max \left(\left(h^{x}\right)^{3},\left(h^{y}\right)^{3}\right)\right) .
\end{aligned}
$$

Lemma 5.5. Let ${ }^{t} \bar{F}_{k l}^{m n}(f),{ }^{t} \bar{F}_{k l}^{m n}(g)$ be the ${ }^{t} \bar{F}^{m n}$-transform components of $f$ and $g$,

$$
\begin{aligned}
{ }^{t} \bar{F}_{k l}^{m n}(f) & =\sum_{s=0}^{m} \sum_{t=0}^{n} a_{(k, l)}^{(s, t)}(f) \mathcal{U}_{(k, l)}^{(s, t)}+\sum_{s=1}^{m} \sum_{t=1}^{n} b_{(k, l)}^{(s, t)}(f) \mathcal{V}_{(k, l)}^{(s, t)}+\sum_{s=0}^{m} \sum_{t=1}^{n} c_{(k, l)}^{(s, t)}(f) \mathcal{X}_{(k, l)}^{(s, t)}+\sum_{s=1}^{m} \sum_{t=0}^{n} d_{(k, l)}^{(s, t)}(f) \mathcal{Y}_{(k, l)}^{(s, t)} \\
{ }^{t} \bar{F}_{k l}^{m n}(g) & =\sum_{s=0}^{m} \sum_{t=0}^{n} a_{(k, l)}^{(s, t)}(g) \mathcal{U}_{(k, l)}^{(s, t)}+\sum_{s=1}^{m} \sum_{t=1}^{n} b_{(k, l)}^{(s, t)}(g) \mathcal{V}_{(k, l)}^{(s, t)}+\sum_{s=0}^{m} \sum_{t=1}^{n} c_{(k, l)}^{(s, t)}(g) \mathcal{X}_{(k, l)}^{(s, t)}+\sum_{s=1}^{m} \sum_{t=0}^{n} d_{(k, l)}^{(s, t)}(g) \mathcal{Y}_{(k, l)}^{(s, t)}
\end{aligned}
$$

Then,

$$
{ }^{t} \bar{F}_{k l}^{m n}(f g)=\sum_{s=0}^{m} \sum_{t=0}^{n} a_{(k, l)}^{(s, t)}(f g) \mathcal{U}_{(k, l)}^{(s, t)}+\sum_{s=1}^{m} \sum_{t=1}^{n} b_{(k, l)}^{(s, t)}(f g) \mathcal{V}_{(k, l)}^{(s, t)}+\sum_{s=0}^{m} \sum_{t=1}^{n} c_{(k, l)}^{(s, t)}(f g) \mathcal{X}_{(k, l)}^{(s, t)}+\sum_{s=1}^{m} \sum_{t=0}^{n} d_{(k, l)}^{(s, t)}(f g) \mathcal{Y}_{(k, l)}^{(s, t)}
$$

where

$$
a_{(k, l)}^{(0,0)}(f g)=a_{(k, l)}^{(0,0)}(f) a_{(k, l)}^{(0,0)}(g)+O\left(\max \left(\left(h^{x}\right)^{3},\left(h^{y}\right)^{3}\right)\right)
$$

for $s=1, \ldots, m$

$$
\begin{aligned}
& a_{(k, l)}^{(s, 0)}(f g)=a_{(k, l)}^{(s, 0)}(f) a_{(k, l)}^{(0,0)}(g)+O\left(\max \left(\left(h^{x}\right)^{3},\left(h^{y}\right)^{3}\right)\right), \\
& d_{(k, l)}^{(s, 0)}(f g)=d_{(k, l)}^{(s, 0)}(f) a_{(k, l)}^{(0,0)}(g)+d_{(k, l)}^{(s, 0)}(g) a_{(k, l)}^{(0,0)}(f)+O\left(\max \left(\left(h^{x}\right)^{3},\left(h^{y}\right)^{3}\right)\right),
\end{aligned}
$$

for $t=1, \ldots, n$

$$
\begin{aligned}
& a_{(k, l)}^{(0, t)}(f g)=a_{(k, l)}^{(0, t)}(f) a_{(k, l)}^{(0,0)}(g)+O\left(\max \left(\left(h^{x}\right)^{3},\left(h^{y}\right)^{3}\right)\right), \\
& c_{(k, l)}^{(0, t)}(f g)=c_{(k, l)}^{(0, t)}(f) a_{(k, l)}^{(0,0)}(g)+c_{(k, l)}^{(0, t)}(g) a_{(k, l)}^{(0,0)}(f)+O\left(\max \left(\left(h^{x}\right)^{3},\left(h^{y}\right)^{3}\right)\right),
\end{aligned}
$$




$$
\begin{aligned}
& \text { for } s=1, \ldots, m, t=1, \ldots, n \\
& \qquad \begin{aligned}
a_{(k, l)}^{(s, t)}(f g) & =a_{(k, l)}^{(s, t)}(f) a_{(k, l)}^{(0,0)}(g)+O\left(\max \left(\left(h^{x}\right)^{3},\left(h^{y}\right)^{3}\right)\right), \\
c_{(k, l)}^{(s, t)}(f g) & =c_{(k, l)}^{(s, t)}(f) a_{(k, l)}^{(0,0)}(g)+c_{(k, l)}^{(s, t)}(g) a_{(k, l)}^{(0,0)}(f)+O\left(\max \left(\left(h^{x}\right)^{3},\left(h^{y}\right)^{3}\right)\right), \\
d_{(k, l)}^{(s, t)}(f g) & =d_{(k, l)}^{(s, t)}(f) a_{(k, l)}^{(0,0)}(g)+d_{(k, l)}^{(s, t)}(g) a_{(k, l)}^{(0,0)}(f)+O\left(\max \left(\left(h^{x}\right)^{3},\left(h^{y}\right)^{3}\right)\right), \\
b_{(k, l)}^{(s, t)}(f g) & =b_{(k, l)}^{(s, t)}(f) a_{(k, l)}^{(0,0)}(g)+c_{(k, l)}^{(0, t)}(f) d_{(k, l)}^{(s, 0)}(g)+c_{(k, l)}^{(0, t)}(g) d_{(k, l)}^{(s, 0)}(f)+b_{(k, l)}^{(s, t)}(g) a_{(k, l)}^{(0,0)}(f) \\
& +O\left(\max \left(\left(h^{x}\right)^{3},\left(h^{y}\right)^{3}\right)\right) .
\end{aligned}
\end{aligned}
$$

Proof. For reasons of brevity, we just prove Eq. (5.12) and the others are proved similarly. We approximate Eq. (4.11) with the help of the trapezium formula as follows

$$
d_{(k, l)}^{(s, t)}(f)=h^{x}\left(\frac{s}{s+1} \sin \frac{(s+1) \pi}{2}+\sin \frac{s \pi}{2}\right) \frac{\partial f\left(x_{k}, y_{l}\right)}{\partial x}+O\left(\max \left(\left(h^{x}\right)^{3},\left(h^{y}\right)^{3}\right)\right) .
$$

On the other hand, from Theorem 5.1 we have

$$
a_{(k, l)}^{(0,0)}=f\left(x_{k}, y_{l}\right)+O\left(\max \left(\left(h^{x}\right)^{3},\left(h^{y}\right)^{3}\right)\right) .
$$

Utilizing the above achievements, we can deduce

$$
\begin{aligned}
d_{(k, l)}^{(s, t)}(f g) & =h^{x}\left(\frac{s}{s+1} \sin \frac{(s+1) \pi}{2}+\sin \frac{s \pi}{2}\right) \frac{\partial\left(f\left(x_{k}, y_{l}\right) g\left(x_{k}, y_{l}\right)\right)}{\partial x}+O\left(\max \left(\left(h^{x}\right)^{3},\left(h^{y}\right)^{3}\right)\right) \\
& =h^{x}\left(\frac{s}{s+1} \sin \frac{(s+1) \pi}{2}+\sin \frac{s \pi}{2}\right)\left(\frac{\partial f\left(x_{k}, y_{l}\right)}{\partial x} g\left(x_{k}, y_{l}\right)+\frac{\partial g\left(x_{k}, y_{l}\right)}{\partial x} f\left(x_{k}, y_{l}\right)\right)+O\left(\max \left(\left(h^{x}\right)^{3},\left(h^{y}\right)^{3}\right)\right) \\
& =\left(d_{(k, l)}^{(s, t)}(f)+O\left(\max \left(\left(h^{x}\right)^{3},\left(h^{y}\right)^{3}\right)\right)\right)\left(a_{(k, l)}^{(0,0)}(g)+O\left(\max \left(\left(h^{x}\right)^{3},\left(h^{y}\right)^{3}\right)\right)\right) \\
& +\left(d_{(k, l)}^{(s, t)}(g)+O\left(\max \left(\left(h^{x}\right)^{3},\left(h^{y}\right)^{3}\right)\right)\right)\left(a_{(k, l)}^{(0,0)}(f)+O\left(\max \left(\left(h^{x}\right)^{3},\left(h^{y}\right)^{3}\right)\right)\right) \\
& =d_{(k, l)}^{(s, t)}(f) a_{(k, l)}^{(0,0)}(g)+d_{(k, l)}^{(s, t)}(g) a_{(k, l)}^{(0,0)}(f)+O\left(\max \left(\left(h^{x}\right)^{3},\left(h^{y}\right)^{3}\right)\right)
\end{aligned}
$$

\subsection{Examples}

In this subsection, we give some examples and we plot the error function $E(x, y)=\left|f(x, y)-{ }^{t} \overline{\hat{f}}_{p q}^{m n}(f(x, y))\right|$ to show that accuracy of approximation by the inverse ${ }^{t} \bar{F}^{m n}$-transform of given two-variable functions. We are going to approximate these functions applying the inverse ${ }^{t} \bar{F}^{m n}$-transform for various amounts of $h^{x}, h^{y}, m$, and $n$.

Example 5.6. In this example, we consider $f(x, y)=\sin (x y)$ on $[0,1] \times[0,1]$. We can find an extension of $f$ such that $\bar{f}$ is four times continuously differentiable. We illustrate $E(x, y)$ in Fig. 3(a), 3(b), and 3(c) for various amounts of $h^{x}, h^{y}, m$, and $n$. These figures show that if we decrease $h^{x}, h^{y}$ and increase $m$ and $n$, then the error function decrease. It worth nothing that the influence of the growth of $m$ and $n$ leads to more reduction of the error function. Moreover, the approximation error of $f$ without extending the inverse ${ }^{t} F^{m n}$-transform in Fig. 3(d) has been plotted. It illustrates that the error along the end lines is more than the extended case. 


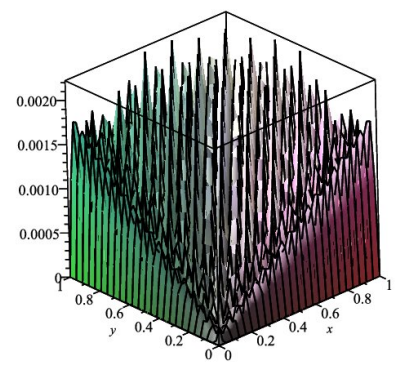

(a) $h^{x}=h^{y}=0.1, p=q=5$

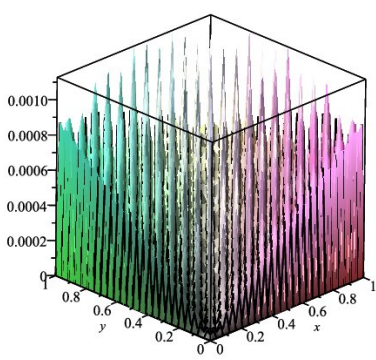

(b) $h^{x}=h^{y}=0.05, p=q=5$

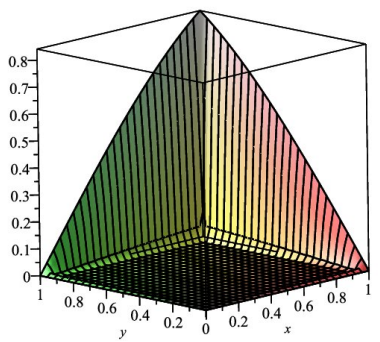

(d) $h^{x}=h^{y}=0.1, p=q=5$

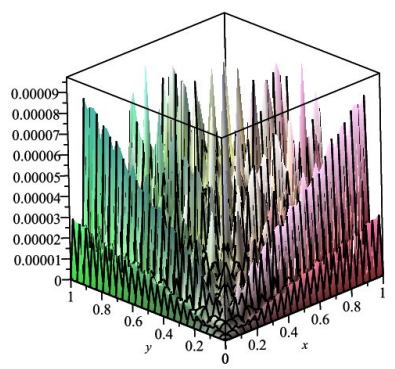

(c) $h^{x}=h^{y}=0.1, p=q=10$

Figure 3: Plot of the absolute error for Example 5.6.

Example 5.7. Consider $f(x, y)=\cos (10 \pi x) \cos (10 \pi y)$ on $[0,1] \times[0,1] . \bar{f}$ is considered the same as $f$ on $\left[-h^{x}, 1+h^{x}\right] \times\left[-h^{y}, 1+h^{y}\right]$ such that $\bar{f}$ is four times continuously differentiable. We report the error function of this example in Fig. 4. Because of the structure of basis functions, in Fig. 4(a), 4(c) we see that the error function has the least value in the case $h^{x}=h^{y}=0.1$ at the middle points of $[0,1] \times[0,1]$. Also, with $h^{x}=h^{y}=0.1$ along with increasing $m$ and $n$, we can improve the approximation on whole of domain.

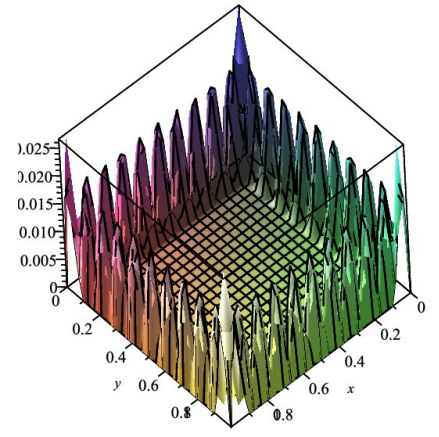

(a) $h^{x}=h^{y}=0.1, p=q=5$

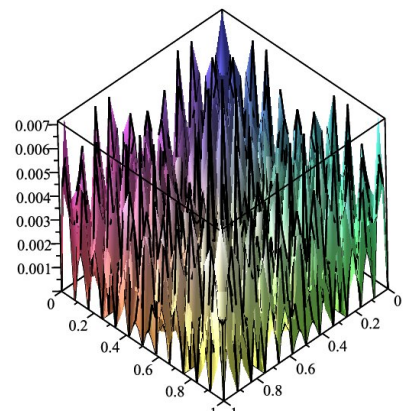

(b) $h^{x}=h^{y}=0.05, p=q=5$

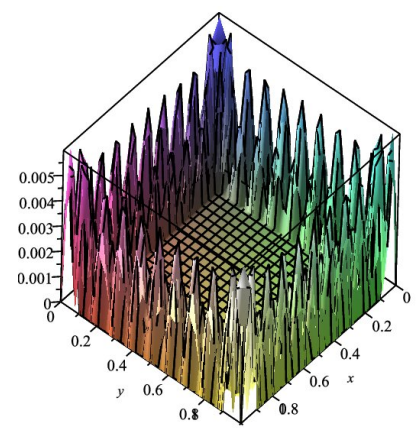

(c) $h^{x}=h^{y}=0.1, p=q=10$

Figure 4: Plot of the absolute error for Example 5.7.

Example 5.8. Consider $f(x, y)=\sqrt{x y}+\sqrt{1-x y}$ on $[0,1] \times[0,1]$. For this function, we can find an extension of $f$ such that it is once continuously differentiable. Therefore, we use the error analysis established in this section for the interior points of $[0,1] \times[0,1]$. We report the error function of this example in Fig. 5. 


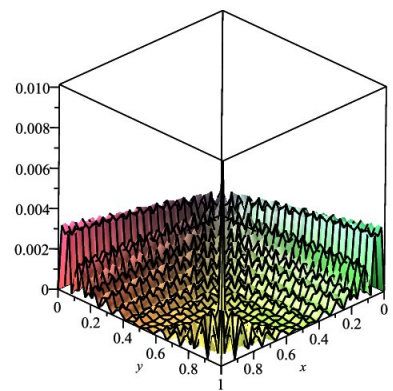

(a) $h^{x}=h^{y}=0.1, p=q=5$

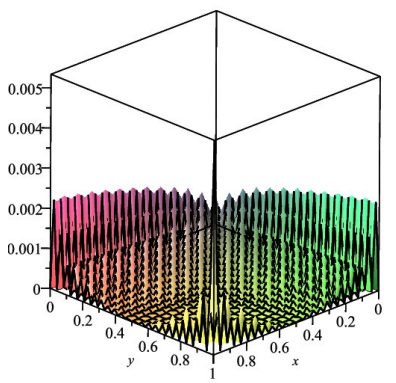

(b) $h^{x}=h^{y}=0.05, p=q=5$

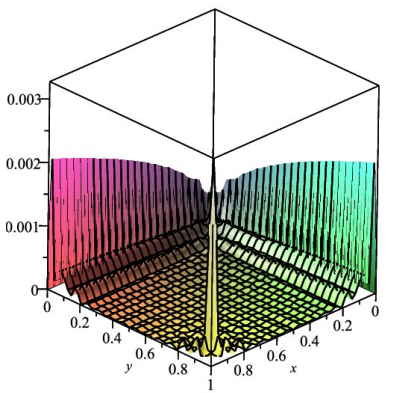

(c) $h^{x}=h^{y}=0.1, p=q=10$

Figure 5: Plot of the absolute error for Example 5.8.

Example 5.9. Consider $f(x, y)=\sin \left(\frac{1}{x y}\right)$ on $(0,1] \times(0,1]$. For this function, we consider $f$ on $[0.11,1] \times$ $[0.11,1] \rightarrow \mathbb{R}$ and $\bar{f}$ the same as $f$ on $\left[0.11-h^{x}, 1+h^{x}\right] \times\left[0.11-h^{y}, 1+h^{y}\right] \rightarrow \mathbb{R}$ such that is four times continuously differentiable. In order to illustrate the established theory, we report the error function of this example in Fig. 6 for the various amounts of $h^{x}, h^{y}, m$, and $n$.

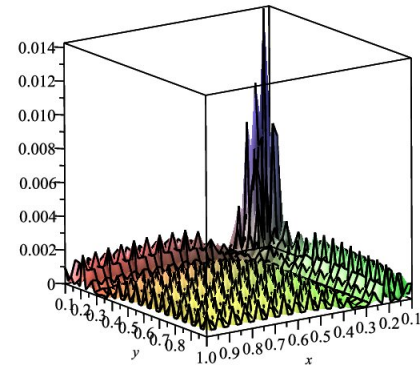

(a) $h^{x}=h^{y}=0.1, p=q=10$

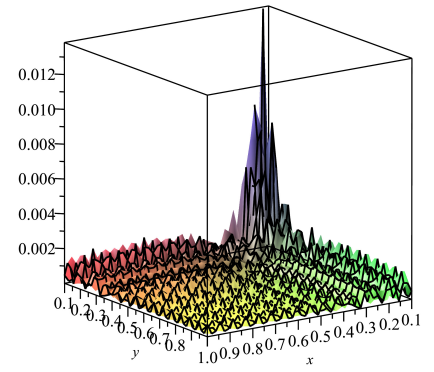

(b) $h^{x}=h^{y}=0.06, p=q=10$

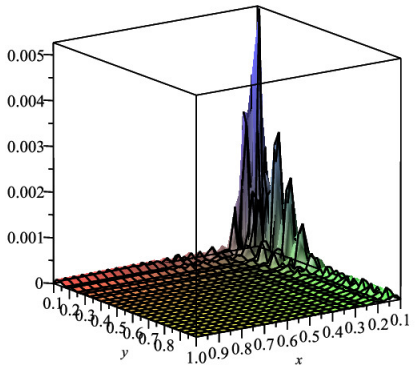

(c) $h^{x}=h^{y}=0.1, p=q=15$

Figure 6: Plot of the absolute error for Example 5.9.

\section{Application of the ${ }^{t} \bar{F}^{m n}$-transform to partial differential equa- tions}

In this section, we are going to apply the ${ }^{t} \bar{F}^{m n}$-transform in order to solve the Cauchy problem of the transport equation. For this purpose, some direct formulas for the ${ }^{t} \bar{F}^{m n}$-transform of the partial derivatives of two-variable functions are obtained and they are applied to solving the transport equation.

\subsection{The ${ }^{t} \bar{F}^{m n}$-transform of the partial derivatives of two-variable function}

In this section, we will obtain ${ }^{t} \bar{F}^{m n}$-transform of the partial derivatives of two-variable function $f$ using the components of ${ }^{t} \bar{F}^{m n}(f)$. For simplicity, we assume that $\left\{\phi_{(k, l)}^{(i, j)}\right\}_{i(j)=1}^{m(n)}$ is the set of the trigonometric basis functions of linear subspace $L_{2}^{m n}\left(\bar{A}_{k, l}\right)$ stated in Sec. 4, i.e. $\phi_{(k, l)}^{(i, j)} \in\left\{\mathcal{U}_{(k, l)}^{(i, j)}, \mathcal{V}_{(k, l)}^{(i, j)}, \mathcal{X}_{(k, l)}^{(i, j)}, \mathcal{Y}_{(k, l)}^{(i, j)}\right\}$. On the other hand, we consider 


$$
{ }^{t} \bar{F}_{k l}^{m n}(f)=\sum_{i=1}^{m} \sum_{j=1}^{n} e_{(k, l)}^{(i, j)}(f) \phi_{(k, l)}^{(i, j)}(x, y),
$$

where $e_{(k, l)}^{(i, j)} \in\left\{a_{(k, l)}^{(i, j)}, b_{(k, l)}^{(i, j)}, c_{(k, l)}^{(i, j)}, d_{(k, l)}^{(i, j)}\right\}$.

In the following, some notations which will be used throughout this section are considered.

Notations: Let $\triangle_{x}^{0} f=\triangle_{y}^{0} f=\triangle_{x, y}^{0,0} f=f$. For $k=1, \cdots, p, l=1, \cdots, q, i=1, \cdots m, j=1, \cdots n$ and $m_{1}, n_{1} \in \mathbb{N}$, we consider the following notations

$$
\begin{array}{ll}
\triangle_{x} f(x, y)=f\left(x+h^{x}, y\right)-f(x, y), & \triangle_{x}^{m_{1}} f(x, y)=\triangle_{x}\left(\triangle_{x}^{m_{1}-1} f(x, y)\right), \\
\triangle_{y} f(x, y)=f\left(x, y+h^{y}\right)-f(x, y), & \triangle_{y}^{n_{1}} f(x, y)=\triangle_{y}\left(\triangle_{y}^{n_{1}-1} f(x, y)\right), \\
\triangle_{x, y} f(x, y)=\triangle_{x} \triangle_{y} f(x, y), & \triangle_{x, y}^{m_{1}, n_{1}} f(x, y)=\triangle_{x, y}\left(\triangle_{x, y}^{m_{1}-1, n_{1}-1} f(x, y)\right), \\
\triangle_{x}\left(e_{(k, l)}^{(i, j)}(f(x, y))\right)=e_{(k+1, l)}^{(i, j)}(f(x, y))-e_{(k, l)}^{(i, j)}(f(x, y)), & \\
\triangle_{y}\left(e_{(k, l)}^{(i, j)}(f(x, y))\right)=e_{(k, l+1)}^{(i, j)}(f(x, y))-e_{(k, l)}^{(i, j)}(f(x, y)), & \\
\triangle_{x, y}\left(e_{(k, l)}^{(i, j)}(f(x, y))\right)=\triangle_{x} \triangle_{y}\left(e_{(k, l)}^{(i, j)}(f(x, y))\right) . &
\end{array}
$$

Theorem 6.1. Let $k=1, \cdots, p, l=1, \cdots, q, i=1, \cdots m, j=1, \cdots n, m_{1}, n_{1} \in \mathbb{N}$ and $f: \mathbb{R}^{2} \rightarrow \mathbb{R}$ be continues. Then the following statements for $(x, y) \in[a, b] \times[c, d]$ are satisfied.

$e_{(k, l)}^{(i, j)}\left(\triangle_{x}^{m_{1}} f(x, y)\right)=\triangle_{x}^{m_{1}}\left(e_{(k, l)}^{(i, j)}(f(x, y))\right), \quad e_{(k, l)}^{(i, j)}\left(\triangle_{y}^{n_{1}} f(x, y)\right)=\triangle_{y}^{n_{1}}\left(e_{(k, l)}^{(i, j)}(f(x, y))\right)$,

$e_{(k, l)}^{(i, j)}\left(\triangle_{x, y}^{m_{1}, n_{1}} f(x, y)\right)=\triangle_{x, y}^{m_{1}, n_{1}}\left(e_{(k, l)}^{(i, j)}(f(x, y))\right)$.

Proof. First we prove that $e_{(k, l)}^{(i, j)}\left(\triangle_{x, y} f(x, y)\right)=\triangle_{x, y}\left(e_{(k, l)}^{(i, j)}(f(x, y))\right)$.

$$
\begin{aligned}
e_{(k, l)}^{(i, j)}\left(\triangle_{x, y} f(x, y)\right) & =\frac{<\triangle_{x, y} f, \phi_{(k, l)}^{(i, j)}>_{k, l}}{<\phi_{(k, l)}^{(i, j)}, \phi_{(k, l)}^{(i, j)}>_{k, l}} \\
& =\frac{1}{h^{x} h^{y}\left\|\phi_{(k, l)}^{(i, j)}\right\|_{k, l}^{2}} \int_{y_{l-1}}^{y_{l+1}} \int_{x_{k-1}}^{x_{k+1}}\left(f\left(x+h^{x}, y+h^{y}\right)-f\left(x+h^{x}, y\right)\right. \\
& \left.-f\left(x, y+h^{y}\right)+f(x, y)\right) \phi_{(k, l)}^{(i, j)}(x, y) \bar{A}_{k, l}(x, y) d x d y \\
& =\frac{1}{h^{x} h^{y}\left\|\phi_{(k, l)}^{(i, j)}\right\|_{k, l}^{2}}\left(\int_{y_{l}}^{y_{l+2}} \int_{x_{k}}^{x_{k+2}} f(x, y) \phi_{(k, l)}^{(i, j)}\left(x-h^{x}, y-h^{y}\right) \bar{A}_{k, l}\left(x-h^{x}, y-h^{y}\right) d x d y\right. \\
& -\int_{y_{l-1}}^{y_{l+1}} \int_{x_{k}}^{x_{k+2}} f(x, y) \phi_{(k, l)}^{(i, j)}\left(x-h^{x}, y\right) \bar{A}_{k, l}\left(x-h^{x}, y\right) d x d y \\
& -\int_{y_{l}}^{y_{l+2}} \int_{x_{k-1}}^{x_{k+1}} f(x, y) \phi_{(k, l)}^{(i, j)}\left(x, y-h^{y}\right) \bar{A}_{k, l}\left(x, y-h^{y}\right) d x d y \\
& \left.+\int_{y_{l-1}}^{y_{l+1}} \int_{x_{k-1}}^{x_{k+1}} f(x, y) \phi_{(k, l)}^{(i, j)}(x, y) \bar{A}_{k, l}(x, y) d x d y\right) \\
& =e_{(k+1, l+1)}^{(i, j)}(f(x, y))-e_{(k+1, l)}^{(i, j)}(f(x, y))-e_{(k, l+1)}^{(i, j)}(f(x, y))+e_{(k, l)}^{(i, j)}(f(x, y)) \\
& =\triangle_{x, y}\left(e_{(k, l)}^{(i, j)}(f(x, y))\right) .
\end{aligned}
$$

In a similar way, we can show that $e_{(k, l)}^{(i, j)}\left(\triangle_{x} f(x, y)\right)=\triangle_{x}\left(e_{(k, l)}^{(i, j)}(f(x, y))\right)$ and $e_{(k, l)}^{(i, j)}\left(\triangle_{y} f(x, y)\right)=$ $\triangle_{y}\left(e_{(k, l)}^{(i, j)}(f(x, y))\right)$. 
The double mathematics induction is used in order to prove $e_{(k, l)}^{(i, j)}\left(\triangle_{x, y}^{m_{1}, n_{1}} f(x, y)\right)=\triangle_{x, y}^{m_{1}, n_{1}}\left(e_{(k, l)}^{(i, j)}(f(x, y))\right)$. We consider the mathematics induction hypothesis on $m_{1}$

$$
e_{(k, l)}^{(i, j)}\left(\triangle_{x, y}^{m_{1}, 1} f(x, y)\right)=\triangle_{x, y}^{m_{1}, 1}\left(e_{(k, l)}^{(i, j)}(f(x, y))\right) .
$$

Then, we conclude

$e_{(k, l)}^{(i, j)}\left(\triangle_{x, y}^{m_{1}+1,1} f(x, y)\right)=e_{(k, l)}^{(i, j)}\left(\triangle_{x}\left(\triangle_{x, y}^{m_{1}, 1} f(x, y)\right)\right)=\triangle_{x}\left(\triangle_{x, y}^{m_{1}, 1}\left(e_{(k, l)}^{(i, j)}(f(x, y))\right)\right)=\triangle_{x, y}^{m_{1}+1,1}\left(e_{k, l)}^{(i, j)}(f(x, y))\right)$.

Similarly, we have $e_{(k, l)}^{(i, j)}\left(\triangle_{x, y}^{1, n_{1}} f(x, y)\right)=\triangle_{x, y}^{1, n_{1}}\left(e_{(k, l)}^{(i, j)}(f(x, y))\right)$. Now we assume for $m_{1}, n_{1} \in \mathbb{N}$

$$
\begin{aligned}
& e_{(k, l)}^{(i, j)}\left(\triangle_{x, y}^{m_{1}+1, n_{1}} f(x, y)\right)=\triangle_{x, y}^{m_{1}+1, n_{1}}\left(e_{(k, l)}^{(i, j)}(f(x, y))\right), \\
& e_{(k, l)}^{(i, j)}\left(\triangle_{x, y}^{m_{1}, n_{1}+1} f(x, y)\right)=\triangle_{x, y}^{m_{1}, n_{1}+1}\left(e_{(k, l)}^{(i, j)}(f(x, y))\right) .
\end{aligned}
$$

We claim that $e_{(k, l)}^{(i, j)}\left(\triangle_{x, y}^{m_{1}+1, n_{1}+1} f(x, y)\right)=\triangle_{x, y}^{m_{1}+1, n_{1}+1}\left(e_{(k, l)}^{(i, j)}(f(x, y))\right)$. For this purpose, we have

$$
\begin{aligned}
e_{(k, l)}^{(i, j)}\left(\triangle_{x, y}^{m_{1}+1, n_{1}+1} f(x, y)\right) & =\frac{<\triangle_{x, y}^{m_{1}+1, n_{1}+1} f, \phi_{(k, l)}^{(i, j)}>_{k, l}}{<\phi_{(k, l)}^{(i, j)}, \phi_{(k, l)}^{(i, j)}>_{k, l}} \\
& =\frac{1}{h^{x} h^{y}\left\|\phi_{(k, l)}^{(i, j)}\right\|_{k, l}^{2}} \int_{y_{l-1}}^{y_{l+1}} \int_{x_{k-1}}^{x_{k+1}}\left(\triangle_{x, y}^{m_{1}, n_{1}} f\left(x+h^{x}, y+h^{y}\right)-\triangle_{x, y}^{m_{1}, n_{1}} f\left(x+h^{x}, y\right)\right. \\
& \left.-\triangle_{x, y}^{m_{1}, n_{1}} f\left(x, y+h^{y}\right)+\triangle_{x, y}^{m_{1}, n_{1}} f(x, y)\right) \phi_{(k, l)}^{(i, j)}(x, y) \bar{A}_{k, l}(x, y) d x d y \\
& =e_{(k+1, l+1)}^{(i, j)}\left(\triangle_{x, y}^{m_{1}, n_{1}} f(x, y)\right)-e_{(k+1, l)}^{(i, j)}\left(\triangle_{x, y}^{m_{1}, n_{1}} f(x, y)\right)-e_{(k, l+1)}^{(i, j)}\left(\triangle_{x, y}^{m_{1}, n_{1}} f(x, y)\right) \\
& +e_{(k, l)}^{(i, j)}\left(\triangle_{x, y}^{m_{1}, n_{1}} f(x, y)\right)=\triangle_{x, y}^{m_{1}+1, n_{1}+1}\left(e_{(k, l)}^{(i, j)}(f(x, y))\right) .
\end{aligned}
$$

This completes the claim. Similarly, we can prove

$$
\begin{gathered}
e_{(k, l)}^{(i, j)}\left(\triangle_{x}^{m_{1}} f(x, y)\right)=\triangle_{x}^{m_{1}}\left(e_{(k, l)}^{(i, j)}(f(x, y))\right), \\
e_{(k, l)}^{(i, j)}\left(\triangle_{y}^{n_{1}} f(x, y)\right)=\triangle_{y}^{n_{1}}\left(e_{(k, l)}^{(i, j)}(f(x, y))\right) .
\end{gathered}
$$

Theorem 6.2. Let $\left\{\phi_{(k, l)}^{(i, j)}\right\}_{i(j)=1}^{m(n)}$ be the trigonometric basis functions and $f$ be sufficiently differentiable. Moreover, let the ${ }^{t} \bar{F}^{m n}$-transform of the partial derivatives of $f$ be given as follows

$$
{ }^{t} \bar{F}^{m n}\left(\frac{\partial^{m_{1}} f}{\partial x^{m_{1}}}\right)=\left[{ }^{t} \bar{F}_{k l}^{m n}\left(\frac{\partial^{m_{1}} f}{\partial x^{m_{1}}}\right)\right]_{p \times q}, \quad \quad{ }^{t} \bar{F}^{m n}\left(\frac{\partial^{n_{1}} f}{\partial y^{n_{1}}}\right)=\left[{ }^{t} \bar{F}_{k l}^{m n}\left(\frac{\partial^{n_{1}} f}{\partial y^{n_{1}}}\right)\right]_{p \times q},
$$

${ }^{t} \bar{F}^{m n}\left(\frac{\partial^{m_{1}+n_{1}} f}{\partial x^{m_{1}} \partial y^{n_{1}}}\right)=\left[{ }^{t} \bar{F}_{k l}^{m n}\left(\frac{\partial^{m_{1}+n_{1}} f}{\partial x^{m_{1}} \partial y^{n_{1}}}\right)\right]_{p \times q}$,

where for $m_{1}, n_{1} \in \mathbb{N}$

$$
\begin{aligned}
& { }^{t} \bar{F}_{k l}^{m n}\left(\frac{\partial^{m_{1}} f}{\partial x^{m_{1}}}\right)=\sum_{i=1}^{m} \sum_{j=1}^{n} e_{(k, l)}^{(i, j)}\left(\frac{\partial^{m_{1}} f}{\partial x^{m_{1}}}\right) \phi_{(k, l)}^{(i, j)}(x, y), \\
& { }^{t} \bar{F}_{k l}^{m n}\left(\frac{\partial^{n_{1}} f}{\partial y^{n_{1}}}\right)=\sum_{i=1}^{m} \sum_{j=1}^{n} e_{(k, l)}^{(i, j)}\left(\frac{\partial^{n_{1}} f}{\partial y^{n_{1}}}\right) \phi_{(k, l)}^{(i, j)}(x, y), \\
& { }^{t} \bar{F}_{k l}^{m n}\left(\frac{\partial^{m_{1}+n_{1}} f}{\partial x^{m_{1}} \partial y^{n_{1}}}\right)=\sum_{i=1}^{m} \sum_{j=1}^{n} e_{(k, l)}^{(i, j)}\left(\frac{\partial^{m_{1}+n_{1}} f}{\partial x^{m_{1}} \partial y^{n_{1}}}\right) \phi_{(k, l)}^{(i, j)}(x, y) .
\end{aligned}
$$


Then for $k=1, \cdots, p, l=1, \cdots, q$, we have

$$
\begin{gathered}
e_{(k, l)}^{(i, j)}\left(\frac{\partial^{m_{1}} f}{\partial x^{m_{1}}}\right)=\frac{1}{\left(h^{x}\right)^{m_{1}}}\left(a_{1} \triangle_{x}\left(e_{(k, l)}^{(i, j)}(f)\right)+a_{2} \triangle_{x}^{2}\left(e_{(k, l)}^{(i, j)}(f)\right)+a_{3} \triangle_{x}^{3}\left(e_{(k, l)}^{(i, j)}(f)\right)+a_{4} \triangle_{x}^{4}\left(e_{(k, l)}^{(i, j)}(f)\right)+\cdots\right), \\
e_{(k, l)}^{(i, j)}\left(\frac{\partial^{n_{1}} f}{\partial y^{n_{1}}}\right)=\frac{1}{\left(h^{y}\right)^{n_{1}}}\left(b_{1} \triangle_{y}\left(e_{(k, l)}^{(i, j)}(f)\right)+b_{2} \triangle_{y}^{2}\left(e_{(k, l)}^{(i, j)}(f)\right)+b_{3} \triangle_{y}^{3}\left(e_{(k, l)}^{(i, j)}(f)\right)+b_{4} \triangle_{y}^{4}\left(e_{(k, l)}^{(i, j)}(f)\right)+\cdots\right), \\
e_{(k, l)}^{(i, j)}\left(\frac{\partial^{m_{1}+n_{1}} f}{\partial x^{m_{1}} \partial y^{n_{1}}}\right)=\frac{1}{\left(h^{x}\right)^{m_{1}}\left(h^{y}\right)^{n_{1}}}\left(a_{1,1} \triangle_{x, y}^{1,1}\left(e_{(k, l)}^{(i, j)}(f)\right)+a_{2,1} \triangle_{x, y}^{2,1}\left(e_{(k, l)}^{(i, j)}(f)\right)+a_{1,2} \triangle_{x, y}^{1,2}\left(e_{(k, l)}^{(i, j)}(f)\right)\right. \\
\left.+a_{2,2} \triangle_{x, y}^{2,2}\left(e_{(k, l)}^{(i, j)}(f)\right)+a_{3,1} \triangle_{x, y}^{3,1}\left(e_{(k, l)}^{(i, j)}(f)\right)+a_{1,3} \triangle_{x, y}^{1,3}\left(e_{(k, l)}^{(i, j)}(f)\right)+\cdots\right),
\end{gathered}
$$

where

$$
\begin{aligned}
& a_{i_{1}}=\left.\frac{\partial^{m_{1}}}{\partial \theta_{1}^{m_{1}}}\left(\frac{\theta_{1}\left(\theta_{1}-1\right) \cdots\left(\theta_{1}-i_{1}+1\right)}{i_{1} !}\right)\right|_{\theta_{1}=0}, \quad i_{1}=1,2, \cdots \\
& b_{j_{1}}=\left.\frac{\partial^{n_{1}}}{\partial \theta_{2}^{n_{1}}}\left(\frac{\theta_{2}\left(\theta_{2}-1\right) \cdots\left(\theta_{2}-j_{1}+1\right)}{j_{1} !}\right)\right|_{\theta_{2}=0}, \quad j_{1}=1,2, \cdots \\
& a_{i_{1}, j_{1}}=\left.\frac{\partial^{m_{1}+n_{1}}}{\partial \theta_{1}^{m_{1}} \partial \theta_{2}^{n_{1}}}\left(\frac{\theta_{1}\left(\theta_{1}-1\right) \cdots\left(\theta_{1}-i_{1}+1\right) \theta_{2}\left(\theta_{2}-1\right) \cdots\left(\theta_{2}-j_{1}+1\right)}{i_{1} ! j_{1} !}\right)\right|_{\theta_{1}\left(\theta_{2}\right)=0}, \quad i_{1}=1,2, \cdots, j_{1}=1,2, \cdots
\end{aligned}
$$

Proof. Utilizing numerical differentiation, we have

$$
\frac{\partial^{m_{1}+n_{1}} f}{\partial x^{m_{1}} \partial y^{n_{1}}}=\frac{1}{\left(h^{x}\right)^{m_{1}}\left(h^{y}\right)^{n_{1}}}\left(a_{1,1} \triangle_{x, y}^{1,1} f+a_{2,1} \triangle_{x, y}^{2,1} f+a_{1,2} \triangle_{x, y}^{1,2} f+a_{2,2} \triangle_{x, y}^{2,2} f+a_{3,1} \triangle_{x, y}^{3,1} f+a_{1,3} \triangle_{x, y}^{1,3} f+\cdots\right) .
$$

Since $e_{(k, l)}^{(i, j)}$ is linear, the following relation can be conclude immediately

$$
\begin{aligned}
e_{(k, l)}^{(i, j)}\left(\frac{\partial^{m_{1}+n_{1}} f}{\partial x^{m_{1}} \partial y^{n_{1}}}\right) & =\frac{1}{\left(h^{x}\right)^{m_{1}}\left(h^{y}\right)^{n_{1}}}\left(a_{1,1} \triangle_{x, y}^{1,1}\left(e_{(k, l)}^{(i, j)}(f)\right)+a_{2,1} \triangle_{x, y}^{2,1}\left(e_{(k, l)}^{(i, j)}(f)\right)+a_{1,2} \triangle_{x, y}^{1,2}\left(e_{(k, l)}^{(i, j)}(f)\right)\right. \\
& \left.+a_{2,2} \triangle_{x, y}^{2,2}\left(e_{(k, l)}^{(i, j)}(f)\right)+a_{3,1} \triangle_{x, y}^{3,1}\left(e_{(k, l)}^{(i, j)}(f)\right)+a_{1,3} \triangle_{x, y}^{1,3}\left(e_{(k, l)}^{(i, j)}(f)\right)+\cdots\right) .
\end{aligned}
$$

This proves Eq. (6.3). In a similar way, we can obtain Eqs. (6.1)-(6.2).

\subsection{Error analysis for the partial derivatives of $f$ using ${ }^{t} \bar{F}^{m n}$-transform}

By approximating the ${ }^{t} \bar{F}^{m n}$-transform of the partial derivatives of $f$ using the components of ${ }^{t} \bar{F}^{m n}(f)$, we face two sources of error. The first comes from ${ }^{t} \bar{F}^{m n}$-transform of the partial derivative of $f$ as follows

$$
\frac{\partial^{m_{1}+n_{1}} f}{\partial x^{m_{1}} \partial y^{n_{1}}} \approx{ }^{t} \overline{\hat{f}}_{p q}^{m n}\left(\frac{\partial^{m_{1}+n_{1}} f}{\partial x^{m_{1}} \partial y^{n_{1}}}\right)=\sum_{k=1}^{p} \sum_{l=1}^{q}{ }^{t} \bar{F}_{k l}^{m n}\left(\frac{\partial^{m_{1}+n_{1}} f}{\partial x^{m_{1}} \partial y^{n_{1}}}\right) \bar{A}_{k, l}(x, y)
$$

and

$$
\left|\frac{\partial^{m_{1}+n_{1}} f}{\partial x^{m_{1}} \partial y^{n_{1}}}-{ }^{t} \overline{\hat{f}}_{p q}^{m n}\left(\frac{\partial^{m_{1}+n_{1}} f}{\partial x^{m_{1}} \partial y^{n_{1}}}\right)\right|=O\left(\max \left(\left(h^{x}\right)^{3},\left(h^{y}\right)^{3}\right)\right) .
$$

The second comes from the results of Theorem 11(a), that is

$$
\begin{aligned}
& \frac{\partial^{m_{1}} f}{\partial x^{m_{1}}} \approx{ }^{t} \bar{f}_{p q}^{m n}\left(\frac{\partial^{m_{1}} f}{\partial x^{m_{1}}}\right)=\sum_{k=1}^{p} \sum_{l=1}^{q}{ }^{t} \bar{F}_{k l}^{m n}\left(\frac{\partial^{m_{1}} f}{\partial x^{m_{1}}}\right) \bar{A}_{k, l}(x, y), \\
& \frac{\partial^{n_{1}} f}{\partial y^{n_{1}}} \approx{ }^{t} \bar{f}_{p q}^{m n}\left(\frac{\partial^{n_{1}} f}{\partial y^{n_{1}}}\right)=\sum_{k=1}^{p} \sum_{l=1}^{q}{ }^{t} \bar{F}_{k l}^{m n}\left(\frac{\partial^{n_{1}} f}{\partial y^{n_{1}}}\right) \bar{A}_{k, l}(x, y), \\
& \frac{\partial^{m_{1}+n_{1}} f}{\partial x^{m_{1}} \partial y^{n_{1}}} \approx t \bar{f}_{p q}^{m n}\left(\frac{\partial^{m_{1}+n_{1}} f}{\partial x^{m_{1}} \partial y^{n_{1}}}\right)=\sum_{k=1}^{p} \sum_{l=1}^{q}{ }^{t} \bar{F}_{k l}^{m n}\left(\frac{\partial^{m_{1}+n_{1}} f}{\partial x^{m_{1}} \partial y^{n_{1}}}\right) \bar{A}_{k, l}(x, y),
\end{aligned}
$$


where

$$
\begin{aligned}
& { }^{t} \overline{\breve{F}}_{k l}^{m n}\left(\frac{\partial^{m_{1}} f}{\partial x^{m_{1}}}\right)=\sum_{i=1}^{m} \sum_{j=1}^{n} \check{e}_{(k, l)}^{(i, j)}\left(\frac{\partial^{m_{1}} f}{\partial x^{m_{1}}}\right) \phi_{(k, l)}^{(i, j)}(x, y), \quad{ }^{t} \bar{F}_{k l}^{m n}\left(\frac{\partial^{n_{1}} f}{\partial y^{n_{1}}}\right)=\sum_{i=1}^{m} \sum_{j=1}^{n} \check{e}_{(k, l)}^{(i, j)}\left(\frac{\partial^{n_{1}} f}{\partial y^{n_{1}}}\right) \phi_{(k, l)}^{(i, j)}(x, y), \\
& { }^{t} \overline{\breve{F}}_{k l}^{m n}\left(\frac{\partial^{m_{1}+n_{1}} f}{\partial x^{m_{1}} \partial y^{n_{1}}}\right)=\sum_{i=1}^{m} \sum_{j=1}^{n} \check{e}_{(k, l)}^{(i, j)}\left(\frac{\partial^{m_{1}+n_{1}} f}{\partial x^{m_{1}} \partial y^{n_{1}}}\right) \phi_{(k, l)}^{(i, j)}(x, y),
\end{aligned}
$$

and $\check{e}_{(k, l)}^{(i, j)}\left(\frac{\partial^{m_{1} f}}{\partial x^{m_{1}}}\right), \check{e}_{(k, l)}^{(i, j)}\left(\frac{\partial^{n_{1}} f}{\partial y^{n_{1}}}\right), \check{e}_{(k, l)}^{(i, j)}\left(\frac{\partial^{m_{1}+n_{1} f}}{\partial x^{m_{1}} \partial y^{n_{1}}}\right)$ are determined by selecting a finite number of the sentences of Eqs. (6.1)-(6.3). We estimate an error bound of $\left|\frac{\partial^{m_{1}+n_{1}} f}{\partial x^{m_{1}} \partial y^{n_{1}}}-{ }^{t} \bar{f}_{p q}^{m_{n}}\left(\frac{\partial^{m_{1}+n_{1}} f}{\partial x^{m_{1}} \partial y^{n_{1}}}\right)\right|$ as follows

$$
\begin{array}{r}
\left|\frac{\partial^{m_{1}+n_{1}} f}{\partial x^{m_{1}} \partial y^{n_{1}}}-{ }^{t} \bar{f}_{p q}^{m n}\left(\frac{\partial^{m_{1}+n_{1}} f}{\partial x^{m_{1}} \partial y^{n_{1}}}\right)\right| \leq\left|\frac{\partial^{m_{1}+n_{1}} f}{\partial x^{m_{1}} \partial y^{n_{1}}}-{ }^{t} \overline{\hat{f}}_{p q}^{m n}\left(\frac{\partial^{m_{1}+n_{1}} f}{\partial x^{m_{1}} \partial y^{n_{1}}}\right)\right| \\
+\left|{ }^{t} \bar{f}_{p q}^{m n}\left(\frac{\partial^{m_{1}+n_{1}} f}{\partial x^{m_{1}} \partial y^{n_{1}}}\right)-{ }^{t} \overline{\hat{f}}_{p q}^{m n}\left(\frac{\partial^{m_{1}+n_{1}} f}{\partial x^{m_{1}} \partial y^{n_{1}}}\right)\right|,
\end{array}
$$

where

$$
\begin{aligned}
& \left|{ }^{t} \bar{f}_{p q}^{m n}\left(\frac{\partial^{m_{1}+n_{1}} f}{\partial x^{m_{1}} \partial y^{n_{1}}}\right)-{ }^{t} \overline{\hat{f}}_{p q}^{m n}\left(\frac{\partial^{m_{1}+n_{1}} f}{\partial x^{m_{1}} \partial y^{n_{1}}}\right)\right| \leq \sum_{k=1}^{p} \sum_{l=1}^{q}\left|{ }^{t} \bar{F}_{k l}^{m n}\left(\frac{\partial^{m_{1}+n_{1}} f}{\partial x^{m_{1}} \partial y^{n_{1}}}\right)-{ }^{t}{\overline{F_{k l}}}^{m n}\left(\frac{\partial^{m_{1}+n_{1}} f}{\partial x^{m_{1}} \partial y^{n_{1}}}\right)\right| \bar{A}_{k, l}(x, y) \\
& =\sum_{k=1}^{p} \sum_{l=1}^{q} \sum_{i=1}^{m} \sum_{j=1}^{n}\left|\check{e}_{(k, l)}^{(i, j)}\left(\frac{\partial^{m_{1}+n_{1}} f}{\partial x^{m_{1}} \partial y^{n_{1}}}\right)-e_{(k, l)}^{(i, j)}\left(\frac{\partial^{m_{1}+n_{1}} f}{\partial x^{m_{1}} \partial y^{n_{1}}}\right)\right|\left|\phi_{(k, l)}^{(i, j)}(x, y)\right| \bar{A}_{k, l}(x, y) .
\end{aligned}
$$

Since $\phi_{(k, l)}^{(i, j)} \in\left\{\mathcal{U}_{(k, l)}^{(i, j)}, \mathcal{V}_{(k, l)}^{(i, j)}, \mathcal{X}_{(k, l)}^{(i, j)}, \mathcal{Y}_{(k, l)}^{(i, j)}\right\}$, we assume $\left|\phi_{(k, l)}^{(i, j)}(x, y)\right| \leq M, \forall x, y \in[a, b] \times[c, d]$. Depending on the number of sentences in which we select in Eq. (6.3) we have

$$
\left|{ }^{t} \bar{f}_{p q}^{m n}\left(\frac{\partial^{m_{1}+n_{1}} f}{\partial x^{m_{1}} \partial y^{n_{1}}}\right)-{ }^{t} \overline{\hat{f}}_{p q}^{m n}\left(\frac{\partial^{m_{1}+n_{1}} f}{\partial x^{m_{1}} \partial y^{n_{1}}}\right)\right| \leq O\left(\left(h^{x}\right)^{q_{1}}+\left(h^{y}\right)^{q_{2}}\right) \sum_{k=1}^{p} \sum_{l=1}^{q} M \bar{A}_{k, l}(x, y),
$$

where $q_{1}$ and $q_{2}$ depended on the number of sentences of Eq. (6.3). Therefore, we can conclude

$$
\left|\frac{\partial^{m_{1}+n_{1}} f}{\partial x^{m_{1}} \partial y^{n_{1}}}-{ }^{t} \bar{f}_{p q}^{m n}\left(\frac{\partial^{m_{1}+n_{1}} f}{\partial x^{m_{1}} \partial y^{n_{1}}}\right)\right| \leq O\left(\max \left(\left(h^{x}\right)^{3},\left(h^{y}\right)^{3}\right)\right)+O\left(\left(h^{x}\right)^{q_{1}}+\left(h^{y}\right)^{q_{2}}\right) .
$$

In a similar way, we can obtain $\left|\frac{\partial^{m_{1}} f}{\partial x^{m_{1}}}-{ }^{t} \bar{f}_{p q}^{m n}\left(\frac{\partial^{m_{1}} f}{\partial x^{m_{1}}}\right)\right| \leq O\left(\max \left(\left(h^{x}\right)^{3},\left(h^{y}\right)^{3}\right)\right)+O\left(\left(h^{x}\right)^{q_{1}}\right)$ and $\mid \frac{\partial^{n_{1}} f}{\partial y^{n_{1}}}-$ ${ }^{t} \bar{f}_{p q}^{m n}\left(\frac{\partial^{n_{1}} f}{\partial y^{n_{1}}}\right) \mid \leq O\left(\max \left(\left(h^{x}\right)^{3},\left(h^{y}\right)^{3}\right)\right)+O\left(\left(h^{y}\right)^{q_{2}}\right)$.

The details of the ${ }^{t} \bar{F}^{m n}$-transform of the partial derivations of $f$ based on the forward, central, and backward differences w.r.t. $x$ and $y$ can be found in Tables 5,6 and 7 in Appendix A respectively.

\subsection{The approximation solution of the Cauchy problem of the transport equation}

We consider the following transport problem

$$
\begin{array}{rlrl}
u_{t}(x, t) & =-\vartheta(x, t) u_{x}(x, t)+\omega(x, t) u(x, t)+\eta(x, t) & & (x, t) \in[0, \ell] \times[0, T], \\
u(x, 0) & =\sigma(x) & & x \in[0, \ell], \\
u(0, t) & =\delta(t) & t \in[0, T] .
\end{array}
$$

We assume

$$
{ }^{t} \bar{F}^{11}(u)=\left[{ }^{t} \bar{F}_{k l}^{11}(u)\right]_{p \times q},
$$


where

$$
{ }^{t} \bar{F}_{k l}^{11}(u)=\sum_{i=0}^{1} \sum_{j=0}^{1} a_{(k, l)}^{(i, j)}(u) \mathcal{U}_{(k, l)}^{(i, j)}+b_{(k, l)}^{(1,1)}(u) \mathcal{V}_{(k, l)}^{(1,1)}+\sum_{i=0}^{1} c_{(k, l)}^{(i, 1)}(u) \mathcal{X}_{(k, l)}^{(i, 1)}+\sum_{j=0}^{1} d_{(k, l)}^{(1, j)}(u) \mathcal{Y}_{(k, l)}^{(1, j)} .
$$

We apply ${ }^{t} \bar{F}^{11}$-transform to the both sides of Eq. (6.4) as follows

$$
{ }^{t} \bar{F}^{11}\left(u_{t}(x, t)\right)=-{ }^{t} \bar{F}^{11}\left(\vartheta(x, t) u_{x}(x, t)\right)+{ }^{t} \bar{F}^{11}(\omega(x, t) u(x, t))+{ }^{t} \bar{F}^{11}(\eta(x, t)) .
$$

From the initial condition $u(x, 0)=\sigma(x)$ for $k=1, \cdots, p$, we have

$\begin{array}{lll}a_{(k, 1)}^{(0,1)}(u)=a_{(k, 1)}^{(1,1)}(u)=0, & c_{(k, 1)}^{(0,1)}(u)=c_{(k, 1)}^{(1,1)}(u)=0, & b_{(k, 1)}^{(1,1)}(u)=d_{(k, 1)}^{(1,1)}(u)=0, \\ a_{(k, 1)}^{(0,0)}(u)=c_{k, 0}(\sigma(x)), & d_{(k, 1)}^{(1,0)}(u)=d_{k, 1}(\sigma(x)), & a_{(k, 1)}^{(1,0)}(u)=c_{k, 1}(\sigma(x)) .\end{array}$

From the boundary condition $u(0, t)=\delta(t)$ for $l=1,2, \cdots, q$, we get

$\begin{array}{lll}a_{(1, l)}^{(1,0)}(u)=a_{(1, l)}^{(1,1)}(u)=0, & b_{(1, l)}^{(1,1)}(u)=c_{(1, l)}^{(1,1)}(u)=0, & d_{(1, l)}^{(1,0)}(u)=d_{(1, l)}^{(1,1)}(u)=0, \\ a_{(1, l)}^{(0,0)}(u)=c_{l, 0}(\delta(t)), & a_{(1, l)}^{(0,1)}(u)=c_{l, 1}(\delta(t)), & c_{(1, l)}^{(0,1)}(u)=d_{l, 1}(\delta(t)),\end{array}$

From Tables 5 and 7 in Appendix A and Lemma 5.5, we have the following recursive equation

$$
z_{(i, j)}^{(l+1)}=\left(A_{(i, j)}^{(l)}+I\right) z_{(i, j)}^{(l)}+\mathbf{Z}_{(i, j)}^{(l)}, \quad l=1,2, \cdots, q-1, \quad i, j=0,1,
$$

where $r=\frac{h^{t}}{h^{x}}$ and $z_{(i, j)}^{(l)}=\left[a_{(2, l)}^{(i, j)}(u), \cdots, a_{(p, l)}^{(i, j)}(u)\right]$,

$$
A_{(i, j)}^{(l)}=\left(\begin{array}{cccc}
a_{(2, l)}^{(i, j)}\left(h^{t} \omega-r \vartheta\right) & 0 & \ldots & 0 \\
r a_{(3, l)}^{(i, j)}(\vartheta) & \ddots & \ldots & 0 \\
\vdots & \ddots & \ddots & \vdots \\
0 & \cdots & r a_{(p, l)}^{(i, j)}(\vartheta) & a_{(p, l)}^{(i, j)}\left(h^{t} \omega-r \vartheta\right)
\end{array}\right)
$$

and

$$
\mathbf{Z}_{(i, j)}^{(l)}=\left[r a_{(2, l)}^{(i, j)}(\vartheta) a_{(1, l)}^{(i, j)}(u)+h^{t} a_{(2, l)}^{(i, j)}(\eta), h^{t} a_{(3, l)}^{(i, j)}(\eta), \cdots, h^{t} a_{(p, l)}^{(i, j)}(\eta)\right] .
$$

We solve Eq. (6.5) and obtain $z_{(i, j)}^{(l)}$ for $i, j=0,1$ and $l=1,2, \cdots, q$. Similarly, by the results of Tables 5 and 7 in Appendix A and Lemma 5.5, we have

$$
x_{(i, 1)}^{(l+1)}=C_{(i, 1)}^{(l)} z_{(0,0)}^{(l)}+\left(A_{(0,0)}^{(l)}+I\right) x_{(i, 1)}^{(l)}+\mathbf{X}_{(i, 1)}^{(l)}, \quad l=1,2, \cdots, q-1, \quad i=0,1,
$$

where $x_{(i, 1)}^{(l)}=\left[c_{(2, l)}^{(i, 1)}(u), \cdots, c_{(p, l)}^{(i, 1)}(u)\right]$,

$$
C_{(i, 1)}^{(l)}=\left(\begin{array}{cccc}
c_{(2, l)}^{(i, 1)}\left(h^{t} \omega-r \vartheta\right) & 0 & \ldots & 0 \\
r c_{(3, l)}^{(i, 1)}(\vartheta) & \ddots & \ldots & 0 \\
\vdots & \ddots & \ddots & \vdots \\
0 & \cdots & r c_{(p, l)}^{(i, 1)}(\vartheta) & c_{(p, l)}^{(i, 1)}\left(h^{t} \omega-r \vartheta\right)
\end{array}\right)
$$

and

$$
\mathbf{X}_{(i, 1)}^{(l)}=\left[r a_{(2, l)}^{(0,0)}(\vartheta) c_{(1, l)}^{(i, 1)}(u)+r c_{(2, l)}^{(i, 1)}(\vartheta) a_{(1, l)}^{(0,0)}(u)+h^{t} c_{(2, l)}^{(i, 1)}(\eta), h^{t} c_{(3, l)}^{(i, 1)}(\eta), \cdots, h^{t} c_{(p, l)}^{(i, 1)}(\eta)\right] .
$$


Utilizing Tables 5 and 7 in Appendix A and Lemma 5.5, we get

$$
y_{(1, j)}^{(l+1)}=D_{(1, j)}^{(l)} z_{(0,0)}^{(l)}+\left(A_{(0,0)}^{(l)}+I\right) y_{(1, j)}^{(l)}+\mathbf{Y}_{(1, j)}^{(l)}, \quad l=1,2, \cdots, q-1, \quad j=0,1,
$$

where $y_{(1, j)}^{(l)}=\left[d_{(2, l)}^{(1, j)}(u), \cdots, d_{(p, l)}^{(1, j)}(u)\right]$

$$
D_{(1, j)}^{(l)}=\left(\begin{array}{cccc}
d_{(2, l)}^{(1, j)}\left(h^{t} \omega-r \vartheta\right) & 0 & \ldots & 0 \\
r d_{(3, l)}^{(1, j)}(\vartheta) & \ddots & \ldots & 0 \\
\vdots & \ddots & \ddots & \vdots \\
0 & \cdots & r d_{(p, l)}^{(1, j)}(\vartheta) & d_{(p, l)}^{(1, j)}\left(h^{t} \omega-r \vartheta\right)
\end{array}\right)
$$

and

$$
\mathbf{Y}_{(1, j)}^{(l)}=\left[r d_{(2, l)}^{(1, j)}(\vartheta) a_{(1, l)}^{(0,0)}(u)+h^{t} d_{(2, l)}^{(1, j)}(\eta), h^{t} d_{(3, l)}^{(1, j)}(\eta), \cdots, h^{t} d_{(p, l)}^{(1, j)}(\eta)\right] .
$$

Finally, we deduce

$$
v^{(l+1)}=B^{(l)} z_{(0,0)}^{(l)}+C_{(0,1)}^{(l)} y_{(1,0)}^{(l)}+D_{(1,0)}^{(l)} x_{(0,1)}^{(l)}+\left(A_{(0,0)}^{(l)}+I\right) v^{(l)}+\mathbf{V}^{(l)}, \quad l=1,2, \cdots, q-1,
$$

where $v^{(l)}=\left[b_{(2, l)}^{(1,1)}(u), \cdots, b_{(p, l)}^{(1,1)}(u)\right]$,

$$
B^{(l)}=\left(\begin{array}{cccc}
b_{(2, l)}^{(1,1)}\left(h^{t} \omega-r \vartheta\right) & 0 & \ldots & 0 \\
r b_{(3, l)}^{(1,1)}(\vartheta) & \ddots & \ldots & 0 \\
\vdots & \ddots & \ddots & \vdots \\
0 & \cdots & r b_{(p, l)}^{(1,1)}(\vartheta) & b_{(p, l)}^{(1,1)}\left(h^{t} \omega-r \vartheta\right)
\end{array}\right)
$$

and

$$
\mathbf{V}^{(l)}=\left[r b_{(2, l)}^{(1,1)}(\vartheta) a_{(1, l)}^{(0,0)}(u)+r d_{(2, l)}^{(1,0)}(\vartheta) c_{(1, l)}^{(0,1)}(u)+h^{t} b_{(2, l)}^{(1,1)}(\eta), h^{t} b_{(3, l)}^{(1,1)}(\eta), \cdots, h^{t} b_{(p, l)}^{(1,1)}(\eta)\right] .
$$

Applying the above achievements, we solve the reductive equation (6.8) and obtain $v^{(l)}$ for $l=1,2, \cdots, q$. Finally, we approximate $u(x, t)$ as follows

$$
{ }^{t} \hat{\hat{f}}_{p q}^{11}(u(x, t))=\sum_{k=1}^{p} \sum_{l=1}^{q}{ }^{t} \bar{F}_{k l}^{11}(u(x, t)) \bar{A}_{k, l}(x, t) .
$$

In the following, we give some examples and apply the established theory in the previous subsection to obtain the approximative solution of the given problems.

Example 6.3. Consider the following Cauchy equation

$$
u_{t}(x, t)=-\frac{x}{2} u_{x}(x, t)+u(x, t)+\left(x^{4}-x^{2}\right)(t+1) \sin \left(x^{2} t\right)-\cos \left(x^{2} t\right) \quad(x, t) \in\left[0, \frac{1}{2}\right] \times\left[0, \frac{1}{2}\right],
$$

with initial and boundary conditions

$$
\begin{array}{ll}
u(x, 0)=1-x^{2} & 0 \leq x \leq \frac{1}{2} . \\
u(0, t)=1 & 0 \leq t \leq \frac{1}{2} .
\end{array}
$$

The exact solution is $u(x, t)=\left(1-x^{2}\right) \cos \left(x^{2} t\right)$. The numerical results using the ${ }^{t} \bar{F}^{11}$-transform for $p=q=20, p=q=40$ are obtained in Table 1 and compared with the $\bar{F}$-transform with the same $p$ and $q$. 
Table 1: The absolute error for Example 6.3

\begin{tabular}{|c|c|c|c|c|}
\hline$\left(x_{i}, t_{i}\right)$ & $\begin{array}{c}\bar{F} \text {-transform } \\
p=q=20\end{array}$ & $\begin{array}{c}{ }^{t} \bar{F}^{11} \text {-transform } \\
p=q=20\end{array}$ & $\begin{array}{c}\bar{F} \text {-transform } \\
p=q=40\end{array}$ & $\begin{array}{c}{ }^{t} \bar{F}^{11} \text {-transform } \\
p=q=40\end{array}$ \\
\hline$\left(\frac{1}{16}, \frac{1}{16}\right)$ & $4.41 \times 10^{-7}$ & $3.25 \times 10^{-7}$ & $3.6 \times 10^{-8}$ & $3.51 \times 10^{-8}$ \\
\hline$\left(\frac{1}{16}, \frac{3}{16}\right)$ & $4.85 \times 10^{-6}$ & $3 \times 10^{-6}$ & $5.38 \times 10^{-7}$ & $3.33 \times 10^{-7}$ \\
\hline$\left(\frac{1}{16}, \frac{5}{16}\right)$ & $1.43 \times 10^{-5}$ & $4.46 \times 10^{-6}$ & $1.60 \times 10^{-6}$ & $9.42 \times 10^{-7}$ \\
\hline$\left(\frac{1}{16}, \frac{7}{16}\right)$ & $2.90 \times 10^{-5}$ & $1.67 \times 10^{-5}$ & $3.23 \times 10^{-6}$ & $1.86 \times 10^{-6}$ \\
\hline$\left(\frac{3}{16}, \frac{1}{16}\right)$ & $1.81 \times 10^{-6}$ & $9.25 \times 10^{-7}$ & $1.92 \times 10^{-7}$ & $9.98 \times 10^{-8}$ \\
\hline$\left(\frac{3}{16}, \frac{3}{16}\right)$ & $8.97 \times 10^{-6}$ & $7.11 \times 10^{-6}$ & $9.93 \times 10^{-7}$ & $7.89 \times 10^{-7}$ \\
\hline$\left(\frac{3}{16}, \frac{5}{16}\right)$ & $2.91 \times 10^{-5}$ & $2.03 \times 10^{-5}$ & $3.22 \times 10^{-6}$ & $2.25 \times 10^{-6}$ \\
\hline$\left(\frac{3}{16}, \frac{7}{16}\right)$ & $6.19 \times 10^{-5}$ & $4.05 \times 10^{-5}$ & $6.90 \times 10^{-6}$ & $4.51 \times 10^{-6}$ \\
\hline$\left(\frac{5}{16}, \frac{1}{16}\right)$ & $7.57 \times 10^{-6}$ & $1.78 \times 10^{-6}$ & $8.32 \times 10^{-7}$ & $1.95 \times 10^{-7}$ \\
\hline$\left(\frac{5}{16}, \frac{3}{16}\right)$ & $7.71 \times 10^{-6}$ & $7.13 \times 10^{-6}$ & $8.54 \times 10^{-7}$ & $7.91 \times 10^{-7}$ \\
\hline$\left(\frac{5}{16}, \frac{5}{16}\right)$ & $4.67 \times 10^{-5}$ & $1.89 \times 10^{-5}$ & $2.35 \times 10^{-6}$ & $2.19 \times 10^{-6}$ \\
\hline$\left(\frac{5}{16}, \frac{7}{16}\right)$ & $4.77 \times 10^{-5}$ & $3.94 \times 10^{-5}$ & $5.33 \times 10^{-6}$ & $4.08 \times 10^{-6}$ \\
\hline$\left(\frac{7}{16}, \frac{1}{16}\right)$ & $1.97 \times 10^{-5}$ & $2.94 \times 10^{-6}$ & $2.19 \times 10^{-6}$ & $3.26 \times 10^{-7}$ \\
\hline$\left(\frac{7}{16}, \frac{3}{16}\right)$ & $3.4 \times 10^{-6}$ & $1.19 \times 10^{-6}$ & $3.84 \times 10^{-7}$ & $1.34 \times 10^{-7}$ \\
\hline$\left(\frac{7}{16}, \frac{5}{16}\right)$ & $1.15 \times 10^{-5}$ & $1.55 \times 10^{-7}$ & $1.26 \times 10^{-6}$ & $2.49 \times 10^{-8}$ \\
\hline$\left(\frac{7}{16}, \frac{7}{16}\right)$ & $2.55 \times 10^{-5}$ & $3.46 \times 10^{-7}$ & $2.78 \times 10^{-6}$ & $2.14 \times 10^{-8}$ \\
\hline
\end{tabular}

Our numerical results confirm that the accuracy of the solution can be increased by replacing ${ }^{t} \bar{F}^{11}$ transform with $\bar{F}$-transform. Moreover, Fig. 7 shows that the better results can be obtained by using the higher degree ${ }^{t} \bar{F}^{m n}$-transform.

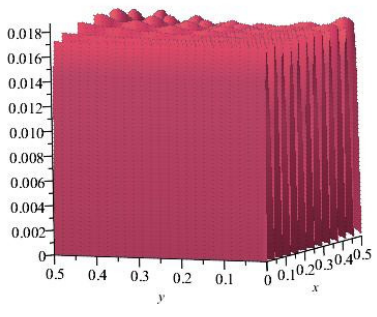

(a) $\quad p=q=5, m=n=1$

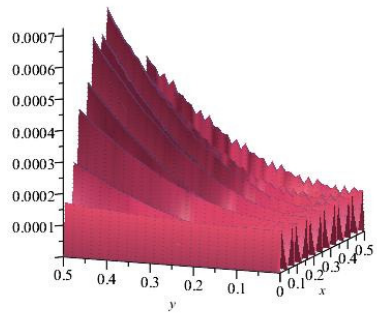

(b) $\quad p=q=5, m=n=5$

Figure 7: Plot of the absolute error for Example 6.3 .

In the following example, to compare the numerical solution obtained by ${ }^{t} \bar{F}^{11}$-transform with the $F^{1}$-transform (see e.g. [27]), we omit some sentences of ${ }^{t} \bar{F}^{11}$-transform and call it ${ }^{t} \bar{F}^{1}$-transform

$$
{ }^{t} \bar{F}^{1}(f)=\left[{ }^{t} \bar{F}_{k l}^{1}(f)\right]_{p \times q},
$$

which the $k l$-component of ${ }^{t} \bar{F}^{1}$ is

$$
{ }^{t} \bar{F}_{k l}^{1}(f)=a_{(k, l)}^{(0,0)} \mathcal{U}_{(k, l)}^{(0,0)}+a_{(k, l)}^{(0,1)} \mathcal{U}_{(k, l)}^{(0,1)}+d_{(k, l)}^{(1,0)} \mathcal{Y}_{(k, l)}^{(1,0)} .
$$

Example 6.4. Let the following transport equation

$$
u_{t}(x, t)=-\frac{2}{3} \sin (x t) u_{x}(x, t)+\frac{\sin (20 \pi t)-\sin (x t) \cos (10 \pi x) \sin (20 \pi x)}{(10 \pi)^{2}}, \quad(x, t) \in\left[0, \frac{1}{2}\right] \times\left[0, \frac{1}{2}\right],
$$


with initial and boundary conditions

$$
\begin{array}{ll}
u(x, 0)=\left(\frac{\cos (10 \pi x)}{10 \pi}\right)^{3} & 0 \leq x \leq \frac{1}{2} . \\
u(0, t)=\frac{1+\sin ^{2}(10 \pi t)}{(10 \pi)^{3}} & 0 \leq t \leq \frac{1}{2} .
\end{array}
$$

The exact solution is $u(x, t)=\frac{\cos ^{3}(10 \pi x)+\sin ^{2}(10 \pi t)}{(10 \pi)^{3}}$.

To illustrate, we give the numerical results using the ${ }^{t} \bar{F}^{11}$-transform, ${ }^{t} \bar{F}^{1}$-transform, and $F^{1}$ transform for $p=q=30$ in Table 2 and Fig. 8 .

Table 2: The absolute error for Example 6.4

\begin{tabular}{|c|c|c|c|}
\hline$\left(x_{i}, t_{i}\right)$ & $F^{1}$-transform & ${ }^{t} \bar{F}^{1}$-transform & ${ }^{t} \bar{F}^{11}$-transform \\
\hline$\left(\frac{1}{15}, \frac{2}{15}\right)$ & $2.60 \times 10^{-4}$ & $1.84 \times 10^{-4}$ & $1.34 \times 10^{-5}$ \\
\hline$\left(\frac{1}{15}, \frac{4}{15}\right)$ & $1.72 \times 10^{-4}$ & $1.23 \times 10^{-4}$ & $1.41 \times 10^{-5}$ \\
\hline$\left(\frac{1}{15}, \frac{6}{15}\right)$ & $4.43 \times 10^{-5}$ & $3.02 \times 10^{-5}$ & $2.32 \times 10^{-6}$ \\
\hline$\left(\frac{3}{15}, \frac{2}{15}\right)$ & $2.16 \times 10^{-4}$ & $1.54 \times 10^{-4}$ & $1.24 \times 10^{-5}$ \\
\hline$\left(\frac{3}{15}, \frac{4}{15}\right)$ & $2.27 \times 10^{-4}$ & $1.46 \times 10^{-4}$ & $1.87 \times 10^{-5}$ \\
\hline$\left(\frac{3}{15}, \frac{6}{15}\right)$ & $3.78 \times 10^{-7}$ & $5.83 \times 10^{-23}$ & $4.85 \times 10^{-24}$ \\
\hline$\left(\frac{5}{15}, \frac{2}{15}\right)$ & $2.75 \times 10^{-4}$ & $1.21 \times 10^{-4}$ & $1.13 \times 10^{-5}$ \\
\hline$\left(\frac{5}{15}, \frac{4}{15}\right)$ & $2.60 \times 10^{-4}$ & $1.74 \times 10^{-4}$ & $1.44 \times 10^{-5}$ \\
\hline$\left(\frac{5}{15}, \frac{6}{15}\right)$ & $4.35 \times 10^{-5}$ & $3.02 \times 10^{-5}$ & $3.01 \times 10^{-6}$ \\
\hline$\left(\frac{7}{15}, \frac{2}{15}\right)$ & $2.62 \times 10^{-4}$ & $1.54 \times 10^{-4}$ & $1.75 \times 10^{-5}$ \\
\hline$\left(\frac{7}{15}, \frac{4}{15}\right)$ & $1.98 \times 10^{-4}$ & $1.11 \times 10^{-4}$ & $1.02 \times 10^{-5}$ \\
\hline$\left(\frac{7}{15}, \frac{6}{15}\right)$ & $4.43 \times 10^{-5}$ & $3 \times 10^{-5}$ & $2.29 \times 10^{-6}$ \\
\hline
\end{tabular}

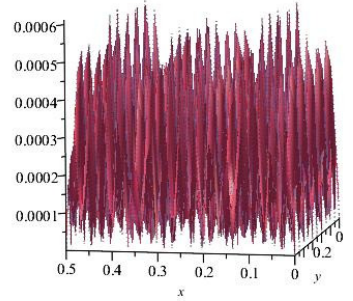

(a) $\quad p=q=30, F^{1}$

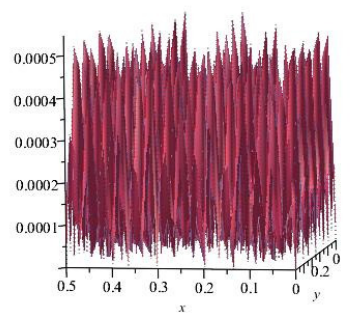

(b) $\quad p=q=30,{ }^{t} \bar{F}^{1}$

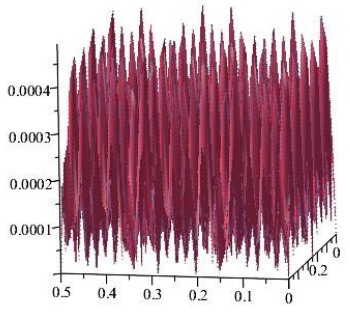

(c) $\quad p=q=30,{ }^{t} \bar{F}^{11}$

Figure 8: Plot of the absolute error for Example 6.4 .

In [27] authors generalized $F$-transform to $F^{1}$-transform with polynomial components. In this example, we show that our numerical results are better than $F^{1}$-transform. Without any bias, we say that the accuracy of some approximation solutions of given problems can be increased by ${ }^{t} \bar{F}^{11}$-transform.

\section{Application of the ${ }^{t} \bar{F}^{m n}$-transform in image compression}

Let $R$ be an array of grey scale image with $P \times Q$ pixels which is discrete two variable function $R: E \rightarrow$ $[0,1]$ is defined over the set of pixels $E=\{(i, j) \mid i=1, \cdots, P, j=1, \cdots, Q\}$ such that $R$ is normalized by dividing the value of each pixel to the length of the gray scale of image. Let $\left(x_{k}, y_{l}\right)$ for $k=1, \ldots, p$, $l=1, \ldots, q,(p, q \geq 2)$ be fixed nodes within $[1, P] \times[1, Q]$ where $1-h^{x}=x_{0}<x_{1}=1<\cdots<x_{p}=$ 
$P<x_{p+1}=P+h^{x}, 1-h^{y}=y_{0}<y_{1}=1<\cdots<y_{q}=Q<y_{q+1}=Q+h^{y}, x_{k}=1+(k-1) h^{x}$ and

$y_{l}=1+(l-1) h^{y}$ such that $h^{x}=\frac{P-1}{p-1}, h^{y}=\frac{Q-1}{q-1}, 2 \leq p \ll P$ and $2 \leq q \ll Q$. We consider $\left[A_{k, l}\right]_{p \times q}$ as the extended sinusoidal shaped basic functions on $\left[x_{0}, x_{p+1}\right] \times\left[y_{0}, y_{q+1}\right]$. To this end, the zero-padding is extended to fill all empty areas of image $R$. We compress image $R$ by ${ }^{t} \bar{F}^{11}$-transform for $k=1, \cdots, p$ and $l=1, \cdots, q$ as follows

$$
{ }^{t} \bar{F}_{k l}^{11}(i, j)=\sum_{s=0}^{1} \sum_{t=0}^{1} a_{(k, l)}^{(s, t)} \mathcal{U}_{(k, l)}^{(s, t)}(i, j)+b_{(k, l)}^{(1,1)} \mathcal{V}_{(k, l)}^{(1,1)}(i, j)+\sum_{s=0}^{1} c_{(k, l)}^{(s, 1)} \mathcal{X}_{(k, l)}^{(s, 1)}(i, j)+\sum_{t=0}^{1} d_{(k, l)}^{(1, t)} \mathcal{Y}_{(k, l)}^{(1, t)}(i, j)
$$

From Lemma 4.4, the coefficients $a_{(k, l)}^{(s, t)}, b_{(k, l)}^{(1,1)}, c_{(k, l)}^{(s, 1)}$, and $d_{(k, l)}^{(1, t)}$ for $s=0,1$ and $t=0,1$ can be obtained by replacing the integrals (4.3)-(4.11) with finite sums as follows

$$
\begin{aligned}
& a_{(k, l)}^{(0,0)}=\frac{1}{h^{x} h^{y}} \sum_{i=1}^{P} \sum_{j=1}^{Q} R(i, j) \bar{A}_{k, l}(i, j), \\
& a_{(k, l)}^{(1,0)}=\frac{4}{h^{x} h^{y}} \sum_{i=1}^{P} \sum_{j=1}^{Q} R(i, j)\left(\cos \left(\frac{\pi}{h^{x}}\left(i-x_{k}\right)\right)-\frac{1}{2}\right) \bar{A}_{k, l}(i, j), \\
& a_{(k, l)}^{(0,1)}=\frac{4}{h^{x} h^{y}} \sum_{i=1}^{P} \sum_{j=1}^{Q} R(i, j)\left(\cos \left(\frac{\pi}{h^{y}}\left(j-y_{l}\right)\right)-\frac{1}{2}\right) \bar{A}_{k, l}(i, j), \\
& a_{(k, l)}^{(1,1)}=\frac{16}{h^{x} h^{y}} \sum_{i=1}^{P} \sum_{j=1}^{Q} R(i, j)\left(\cos \left(\frac{\pi}{h^{x}}\left(i-x_{k}\right)\right)-\frac{1}{2}\right)\left(\cos \left(\frac{\pi}{h^{y}}\left(j-y_{l}\right)\right)-\frac{1}{2}\right) \bar{A}_{k, l}(i, j), \\
& b_{(k, l)}^{(1,1)}=\frac{16}{h^{x} h^{y}} \sum_{i=1}^{P} \sum_{j=1}^{Q} R(i, j) \sin \left(\frac{\pi}{h^{x}}\left(i-x_{k}\right)\right) \sin \left(\frac{\pi}{h^{y}}\left(j-y_{l}\right)\right) \bar{A}_{k, l}(i, j), \\
& c_{(k, l)}^{(0,1)}=\frac{4}{h^{x} h^{y}} \sum_{i=1}^{P} \sum_{j=1}^{Q} R(i, j) \sin \left(\frac{\pi}{h^{y}}\left(j-y_{l}\right)\right) \bar{A}_{k, l}(i, j), \\
& c_{(k, l)}^{(1,1)}=\frac{16}{h^{x} h^{y}} \sum_{i=1}^{P} \sum_{j=1}^{Q} R(i, j)\left(\cos \left(\frac{\pi}{h^{x}}\left(i-x_{k}\right)\right)-\frac{1}{2}\right) \sin \left(\frac{\pi}{h^{y}}\left(j-y_{l}\right)\right) \bar{A}_{k, l}(i, j), \\
& d_{(k, l)}^{(1,0)}=\frac{4}{h^{x} h^{y}} \sum_{i=1}^{P} \sum_{j=1}^{Q} R(i, j) \sin \left(\frac{\pi}{h^{x}}\left(i-x_{k}\right)\right) \bar{A}_{k, l}(i, j), \\
& d_{(k, l)}^{(1,1)}=\frac{16}{h^{x} h^{y}} \sum_{i=1}^{P} \sum_{j=1}^{Q} R(i, j) \sin \left(\frac{\pi}{h^{x}}\left(i-x_{k}\right)\right)\left(\cos \left(\frac{\pi}{h^{y}}\left(j-y_{l}\right)\right)-\frac{1}{2}\right) \bar{A}_{k, l}(i, j) .
\end{aligned}
$$

The compressed image $\left[{ }^{t} \bar{F}_{k, l}^{11}\right]_{p \times q}$ can be decoded by using the inverse ${ }^{t} \bar{F}^{11}$-transform as follows

$$
{ }^{t} \overline{\hat{f}}_{p q}^{11}(R(i, j))=\sum_{k=1}^{p} \sum_{l=1}^{q}{ }^{t} \bar{F}_{k l}^{11}(i, j) \bar{A}_{k, l}(i, j), \quad(i, j) \in\{1, \cdots, P\} \times\{1, \cdots, Q\} .
$$

The value $\rho=\frac{p q}{P Q}$ is called compression rate. The quality of the reconstructed image via the Peak Signal to Noise Ratio can be evaluated by

$$
P S N R=20 \log _{10} \frac{L-1}{R M S E},
$$

where $L$ is the length of the gray scale of image and

$$
R M S E=\sqrt{\frac{\sum_{i=1}^{P} \sum_{j=1}^{Q}\left(R(i, j)-{ }^{t} \hat{\hat{f}}_{p q}^{11}(R(i, j))\right)^{2}}{P Q}},
$$


is Root Mean Square Error.

In this paper, we extract our test images from http://decsai.ugr.es/cvg/dbimagenes/. We apply our method to gray image Zelda (Fig. 9(a)) of size $256 \times 256$. In Fig. 9(b)-9(f), we show decoded images for the set of compression ratio $\rho=\{0.0156,0.0625,0.1406,0.2844,0.3086\}$. The PSNR trends of corresponding images are $23.1204,28.4264,31.9527,33.4949,35.1267$ respectively.

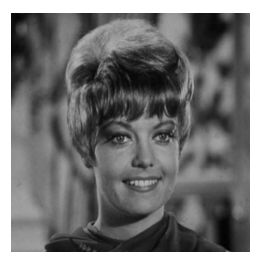

(a)

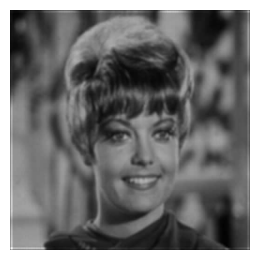

(d)

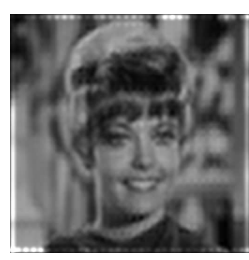

(b)

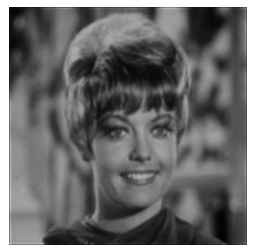

(e)

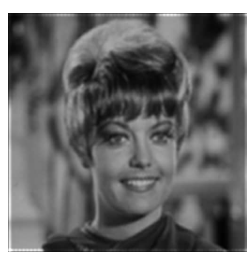

(c)

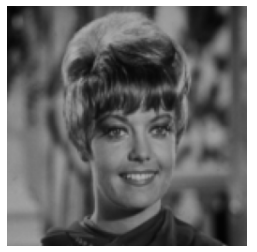

(f)

Figure 9: (a) Source image. (b) ${ }^{t} \bar{F}^{11}$-transform with $\rho=0.0156$. (c) ${ }^{t} \bar{F}^{11}$-transform with $\rho=0.0625$. (d) ${ }^{t} \bar{F}^{11}$-transform with $\rho=0.1406$. (e) ${ }^{t} \bar{F}^{11}$-transform with $\rho=0.2844$. (f) ${ }^{t} \bar{F}^{11}$-transform with $\rho=0.3086$.

To compare ${ }^{t} \bar{F}^{11}$-transform method with $F^{1}$-transform in [27], we show our results for three gray level images as Lena, Einstein and Leopard of size $256 \times 256$ ( Fig. 10(a), 10(b), and 10(c)). For reasons of brevity, we present the mean value of PSNR trends which is obtained from images Lena, Einstein, and Leopard in Table 3.

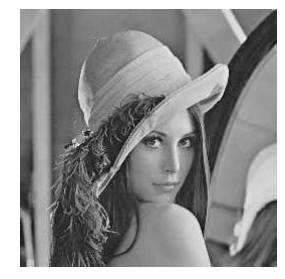

(a)

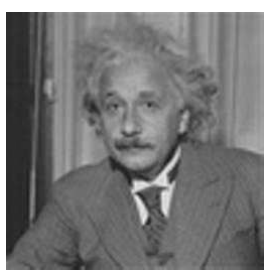

(b)

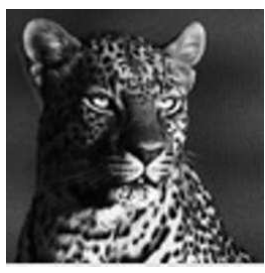

(c)

Figure 10: (a) Lena; (b) Einstein; (c) Leopard. 
Table 3: Mean value of PSNR trends for images Lena, Einstein and Leopard

\begin{tabular}{|c|c|c|}
\hline$\rho$ & $F^{1}$-transform & ${ }^{t} \bar{F}^{11}$-transform \\
\hline 0.062500 & 22.8753 & 25.2342 \\
\hline 0.140625 & 25.4007 & 28.1375 \\
\hline 0.284444 & 28.1530 & 31.0680 \\
\hline 0.390625 & 29.2410 & 31.9800 \\
\hline 0.444444 & 30.2320 & 32.7527 \\
\hline 0.562500 & 31.0130 & 33.5453 \\
\hline
\end{tabular}

Finally, we compare our results with the Fast MF-transform method [28]. So, we evaluate the PSNR and SSIM ( structural similarity index measure) for gray image Airport (Fig. 11(a)) of size $512 \times 512$, that is decoded with compression ratio values 0.06 and 0.11 under ${ }^{t} \bar{F}^{11}$-transform method. The obtained results, which are shown in Fig. 11 and Table 4, verify with less CPU time, we obtain the decoded image which the quality of image is better than the Fast MF-transform method. Also, with the same PSNR value, the CPU time of our method is about half.

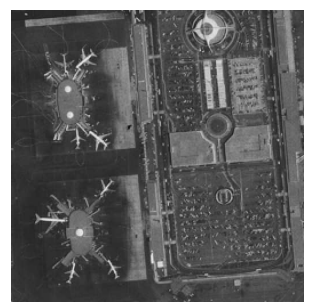

(a)

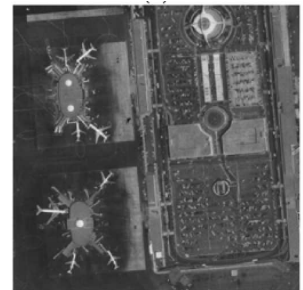

(b)
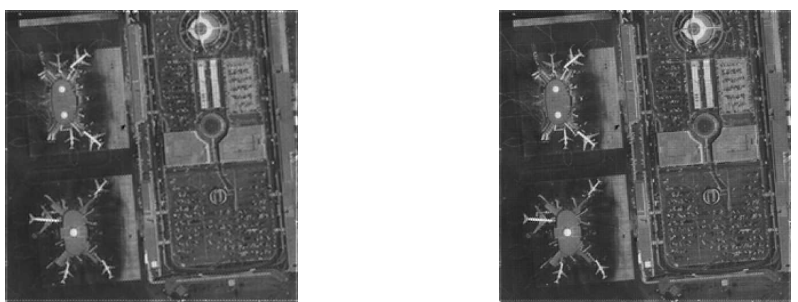

(c) (d)

Figure 11: (a) Source image. (b) Decoded image at level $3(\rho=0.11)$ with Fast MF-tr. (c) Decoded image $(\rho=0.06)$ with ${ }^{t} \bar{F}^{11}$-tr. (d) Decoded image $(\rho=0.11)$ with ${ }^{t} \bar{F}^{11}$-tr.

Table 4: PSNR, SSIM and CPU time trends for the $512 \times 512$ source image $11(\mathrm{a})$

\begin{tabular}{|l|l|c|c|l|c|}
\hline Algorithm & $\rho$ & levels & PSNR & SSIM & CPU time(s) \\
\hline Fast MF-tr & 0.11 & 3 & 28.10 & 0.94 & 59.86 \\
\hline${ }^{t} \bar{F}^{11}$-tr & 0.11 & & 30.57 & 0.95 & 41.14 \\
\hline${ }^{t} F^{11}-\operatorname{tr}$ & 0.06 & & 28.27 & 0.94 & 29.21 \\
\hline
\end{tabular}

As mentioned above, the results obtained by using the ${ }^{t} \bar{F}^{11}$-transform for compression of gray scale images show the efficiency of the proposed method from the point of view of accuracy and computation 
speed. On the other words, the results of Theorem 5.4

$$
R(i, j)-{ }^{t} \overline{\hat{f}}_{p q}^{11}(R(i, j))=O\left(\max \left(\left(h^{x}\right)^{3},\left(h^{y}\right)^{3}\right)\right), \quad(i, j) \in\{1, \cdots, P\} \times\{1, \cdots, Q\},
$$

verify that ${ }^{t} \bar{F}^{11}$-transform speeds up the decoding time by enhancement of the decoded image quality with small compression ratio.

\section{Appendix A}

In the present Appendix, we have proposed the details of the ${ }^{t} \bar{F}^{m n}$-transform of the partial derivatives of two-variable functions based on the forward, central and backward differences with respect to $x$ and $y$.

Table 5: The ${ }^{t} \bar{F}^{m n}$-transform of partial derivations of $f$ based on the forward differences w.r.t. $x$ and $y$

\begin{tabular}{|c|}
\hline $\begin{aligned}{ }^{t} \bar{F}_{k l}^{m n}\left(\frac{\partial f}{\partial x}\right) & =\sum_{s=0}^{m} \sum_{t=0}^{n} \frac{a_{(k+1, l)}^{(s, t)}-a_{(k, l)}^{(s, t)}}{h^{x}} \mathcal{U}_{(k, l)}^{(s, t)}+\sum_{s=1}^{m} \sum_{t=1}^{n} \frac{b_{(k+1, l)}^{(s, t)}-b_{(k, l)}^{(s, t)}}{h^{x}} \mathcal{V}_{(k, l)}^{(s, t)} \\
& +\sum_{s=0}^{m} \sum_{t=1}^{n} \frac{c_{(k+1, l)}^{(s, t)}-c_{(k, l)}^{(s, t)}}{h^{x}} \mathcal{X}_{(k, l)}^{(s, t)}+\sum_{s=1}^{m} \sum_{t=0}^{n} \frac{d_{(k+1, l)}^{(s, t)}-d_{(k, l)}^{(s, t)}}{h^{x}} \mathcal{Y}_{(k, l)}^{(s, t)}+O\left(h^{x}\right)\end{aligned}$ \\
\hline $\begin{aligned}{ }^{t} \bar{F}_{k l}^{m n}\left(\frac{\partial f}{\partial y}\right) & =\sum_{s=0}^{m} \sum_{t=0}^{n} \frac{a_{(k, l+1)}^{(s, t)}-a_{(k, l)}^{(s, t)}}{h^{y}} \mathcal{U}_{(k, l)}^{(s, t)}+\sum_{s=1}^{m} \sum_{t=1}^{n} \frac{b_{(k, l+1)}^{(s, t)}-b_{(k, l)}^{(s, t)}}{h^{y}} \mathcal{V}_{(k, l)}^{(s, t)} \\
& +\sum_{s=0}^{m} \sum_{t=1}^{n} \frac{c_{(k, l+1)}^{(s, t)}-c_{(k, l)}^{(s, t)}}{h^{y}} \mathcal{X}_{(k, l)}^{(s, t)}+\sum_{s=1}^{m} \sum_{t=0}^{n} \frac{d_{(k, l+1)}^{(s, t)}-d_{(k, l)}^{(s, t)}}{h^{y}} \mathcal{Y}_{(k, l)}^{(s, t)}+O\left(h^{y}\right)\end{aligned}$ \\
\hline $\begin{aligned}{ }^{t} \bar{F}_{k l}^{m n}\left(\frac{\partial^{2} f}{\partial x \partial y}\right) & =\sum_{s=0}^{m} \sum_{t=0}^{n} \frac{a_{(k+1, l+1)}^{(s, t)}-a_{(k+1, l)}^{(s, t)}-a_{(k, l+1)}^{(s, t)}+a_{(k, l)}^{(s, t)}}{h^{x} h^{y}} \mathcal{U}_{(k, l)}^{(s, t)} \\
& +\sum_{s=1}^{m} \sum_{t=1}^{n} \frac{b_{(k+1, l+1)}^{(s, t)}-b_{(k+1, l)}^{(s, t)}-b_{(k, l+1)}^{(s, t)}+b_{(k, l)}^{(s, t)}}{h^{x} h^{y}} \mathcal{V}_{(k, l)}^{(s, t)} \\
& +\sum_{s=0}^{m} \sum_{t=1}^{n} \frac{c_{(k+1, l+1)}^{(s, t)}-c_{(k+1, l)}^{(s, t)}-c_{(k, l+1)}^{(s, t)}+c_{(k, l)}^{(s, t)}}{h^{x} h^{y}} \mathcal{X}_{(k, t)}^{(s, t)} \\
& +\sum_{s=1}^{m} \sum_{t=0}^{n} \frac{d_{(k+1, l+1)}^{(s, t)}-d_{(k+1, l)}^{(s, t)}-d_{(k, l+1)}^{(s, t)}+d_{(k, l)}^{(s, t)}}{h^{x} h^{y}} \mathcal{Y}_{(k, l)}^{(s, t)}+O\left(h^{x}+h^{y}\right)\end{aligned}$ \\
\hline 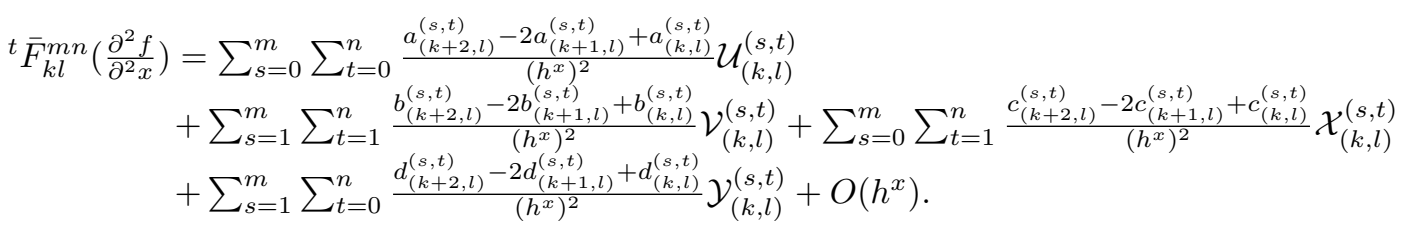 \\
\hline $\begin{aligned}{ }^{t} \bar{F}_{k l}^{m n}\left(\frac{\partial^{2} f}{\partial^{2} y}\right) & =\sum_{s=0}^{m} \sum_{t=0}^{n} \frac{a_{(k, l+2)}^{(s, t)}-2 a_{(k, l+1)}^{(s, t)}+a_{(k, l)}^{(s, t)}}{\left(h^{y}\right)^{2}} \mathcal{U}_{(k, l)}^{(s, t)} \\
& +\sum_{s=1}^{m} \sum_{t=1}^{n} \frac{b_{(k, l+2)}^{(s, t)}-2 b_{(k, t)}^{(s)}+b_{(k, l)}^{(s, t)}}{\left.\left(h^{y}\right)^{2}\right)} \mathcal{V}_{(k, l)}^{(s, t)}+\sum_{s=0}^{m} \sum_{t=1}^{n} \frac{c_{(k, l+2)}^{(s, t)}-2 c_{(k, l+1)}^{(s, t)}+c_{(k, l)}^{(s, t)}}{\left(h^{y}\right)^{2}} \mathcal{X}_{(k, l)}^{(s, t)} \\
& +\sum_{s=1}^{m} \sum_{t=0}^{n} \frac{d_{(k, l+2)}^{(s, t)}-2 d_{(k, l)}^{(s, t)}+d_{(k, l)}^{(s, t)}}{\left(h^{y}\right)^{2}} \mathcal{Y}_{(k, l)}^{(s, t)}+O\left(h^{y}\right) .\end{aligned}$ \\
\hline
\end{tabular}


Table 6: The ${ }^{t} \bar{F}^{m n}$-transform of partial derivations of $f$ based on the central differences w.r.t. $x$ and $y$

\begin{tabular}{|c|}
\hline $\begin{aligned}{ }^{t} \bar{F}_{k l}^{m n}\left(\frac{\partial f}{\partial x}\right) & =\sum_{s=0}^{m} \sum_{t=0}^{n} \frac{a_{(k+1, l)}^{(s, t)}-a_{(k-1, l)}^{(s, t)}}{2 h^{x}} \mathcal{U}_{(k, l)}^{(s, t)}+\sum_{s=1}^{m} \sum_{t=1}^{n} \frac{b_{(k+1, l)}^{(s, t)}-b_{(k-1, l)}^{(s, t)}}{2 h^{x}} \mathcal{V}_{(k, l)}^{(s, t)} \\
& +\sum_{s=0}^{m} \sum_{t=1}^{n} \frac{c_{(k+1, l)}^{(s, l)}-c_{(k-1, l)}^{(s, t)}}{2 h^{x}} \mathcal{X}_{(k, l)}^{(s, t)}+\sum_{s=1}^{m} \sum_{t=0}^{n} \frac{d_{(k+1, l)}^{\left(s,-d_{(k-1, l)}\right.}}{2 h^{x}} \mathcal{Y}_{(k, l)}^{(s, t)}+O\left(\left(h^{x}\right)^{2}\right) .\end{aligned}$ \\
\hline $\begin{aligned}{ }^{t} \bar{F}_{k l}^{m n}\left(\frac{\partial f}{\partial y}\right) & =\sum_{s=0}^{m} \sum_{t=0}^{n} \frac{a_{(k, l+1)}^{(s, t)}-a_{(k, l-1)}^{(s, t)}}{2 h^{y}} \mathcal{U}_{(k, l)}^{(s, t)}+\sum_{s=1}^{m} \sum_{t=1}^{n} \frac{b_{(k, l+1)}^{(s, t)}-b_{(k, l-1)}^{(s, t)}}{2 h^{y}} \mathcal{V}_{(k, l)}^{(s, t)} \\
& +\sum_{s=0}^{m} \sum_{t=1}^{n} \frac{c_{(k, l+1)}^{(s, t)}-c_{(k, l-1)}^{(s, t)}}{2 h^{y}} \mathcal{X}_{(k, l)}^{(s, t)}+\sum_{s=1}^{m} \sum_{t=0}^{n} \frac{d_{(k, l+1)}^{(s, t)}-d_{(k, l-1)}^{(s, t)}}{2 h^{y}} \mathcal{Y}_{(k, l)}^{(s, t)}+O\left(\left(h^{y}\right)^{2}\right) .\end{aligned}$ \\
\hline 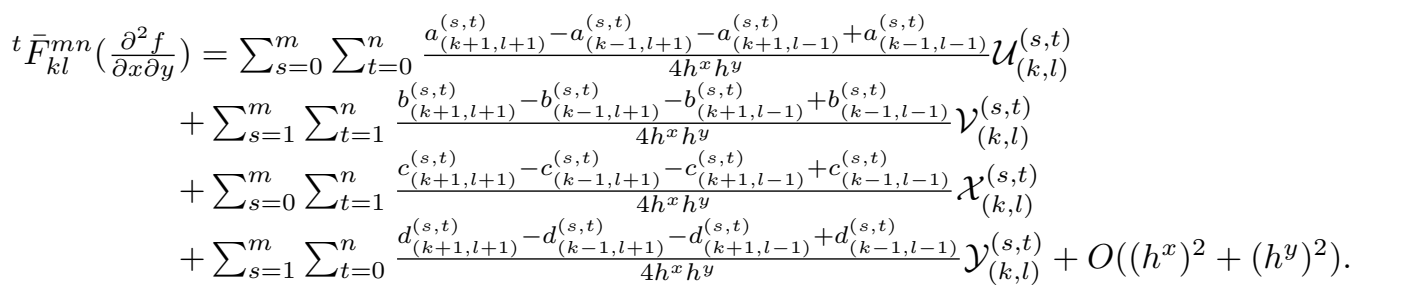 \\
\hline $\begin{aligned}{ }^{t} \bar{F}_{k l}^{m n}\left(\frac{\partial^{2} f}{\partial^{2} x}\right) & =\sum_{s=0}^{m} \sum_{t=0}^{n} \frac{a_{(k+1, l)}^{(s, t)}-2 a_{(k, t)}^{(s, t)}+a_{(k-1, l)}^{(s, t)}}{\left(h^{x}\right)^{2}} \mathcal{U}_{(k, l)}^{(s, t)} \\
& +\sum_{s=1}^{m} \sum_{t=1}^{n} \frac{b_{(k+1, l)}^{(s, t)}-2 b_{k, t)}^{(s, t)}+b_{(k-1, l)}^{(s, t)}}{\left(h^{x}\right)^{2}} \mathcal{V}_{(k, l)}^{(s, t)}+\sum_{s=0}^{m} \sum_{t=1}^{n} \frac{c_{(k+1, l)}^{(s, t)}-2 c_{(k, l)}^{(s, t)}+c_{(k-1, l)}^{(s, t)}}{\left(h^{x}\right)^{2}} \mathcal{X}_{(k, l)}^{(s, t)} \\
& +\sum_{s=1}^{m} \sum_{t=0}^{n} \frac{d_{(k+1, l)}^{(s, t)}-2 d_{(k, l)}^{(s, t)}+d_{(k-1, l)}^{(s, t)}}{\left(h^{x}\right)^{2}} \mathcal{Y}_{(k, l)}^{(s, t)}+O\left(\left(h^{x}\right)^{2}\right) .\end{aligned}$ \\
\hline $\begin{aligned}{ }^{t} \bar{F}_{k l}^{m n}\left(\frac{\partial^{2} f}{\partial^{2} y}\right) & =\sum_{s=0}^{m} \sum_{t=0}^{n} \frac{a_{(k, l+1)}^{(s, t)}-2 a_{(k, l)}^{(s, t)}+a_{(k, l-1)}^{(s, t)}}{\left(h^{y}\right)^{2}} \mathcal{U}_{(k, l)}^{(s, t)} \\
& +\sum_{s=1}^{m} \sum_{t=1}^{n} \frac{b_{(k, l+1)}^{(s, t)}-2 b_{(k, l)}^{(s, t)}+b_{(k, l-1)}^{(s, t)}}{\left(h^{y}\right)^{2}} \mathcal{V}_{(k, t)}^{(s, t)}+\sum_{s=0}^{m} \sum_{t=1}^{n} \frac{c_{(k, l+1)}^{(s, t)}-2 c_{(k, l)}^{(s, t)}+c_{(k, l-1)}^{(s, t)}}{\left(h^{y}\right)^{2}} \mathcal{X}_{(k, l)}^{(s, t)} \\
& +\sum_{s=1}^{m} \sum_{t=0}^{n} \frac{d_{(k, l+1)}^{(s, t)}-2 d_{(k, l)}^{(s, t)}+d_{(k, l-1)}^{(s, t)}}{\left(h^{y}\right)^{2}} \mathcal{Y}_{(k, l)}^{(s, t)}+O\left(\left(h^{y}\right)^{2}\right) .\end{aligned}$ \\
\hline
\end{tabular}


Table 7: The ${ }^{t} \bar{F}^{m n}$-transform of partial derivations of $f$ based on the backward differences w.r.t. $x$ and $y$

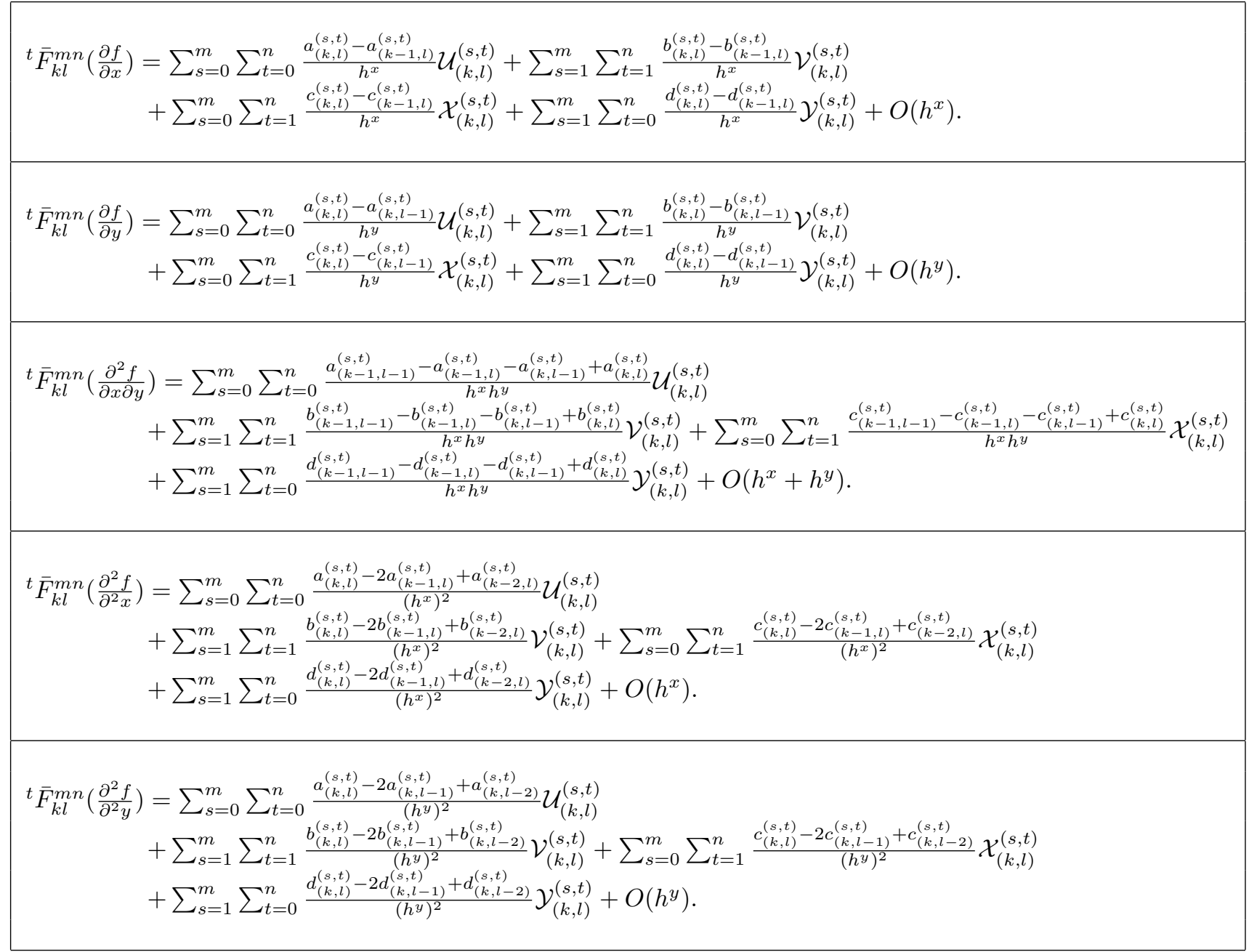

\section{Acknowledgements}

The work of Irina Perfilieva was supported from ERDF/ESF by the project "Centre for the development of Artificial Intelligence Methods for the Automotive Industry of the region" No. CZ.02.1.01/0.0/0.0/17049/0008414.

\section{Compliance with Ethical Standards statements}

Ethical approval: This article does not contain any studies with human participants or animals performed by any of the authors.

Funding details: No funding was received for conducting this study.

Conflict of interest: The authors declare that there is no conflict of interests regarding the publication of this paper.

Informed Consent: The authors agree with the publication of the article in Soft Computing journal Authorship contributions: Writing-original draft - R. Alikhani, M. Ganjeh-Alamdari; Writing-review \& editing, Irina Perfilieva; Numerical Computation - R. Alikhani, M. Ganjeh-Alamdari. 


\section{References}

[1] Perfilieva I (2006) Fuzzy transforms: theory and applications. Fuzzy Sets Syst 157:993-1023

[2] Khastan A, Alijani Z, Perfilieva I (2017) Fuzzy transform to approximate solution of two-point boundary value problems. Math. Methods Appl. Sci 40:6147-6154

[3] Perfilieva I, De Baets B (2010) Fuzzy transforms of monotone functions with applications to image compression. Inf. Sci 180:3304-3315

[4] Perfilieva I (2017) F-transform for numerical solution of two-point boundary value problem. Iranian J. Fuzzy Syst 14:1-13

[5] Tomasiello S (2017) An alternative use of fuzzy transform with application to a class of delay differential equations. Int. J. Comput. Math 94:1719-1726

[6] ALKasasbeh H, Perfilieva I, Ahmad MZ, Yahya ZR (2018) New approximation methods based on fuzzy transform for solving SODEs: II. Applied System Innovation 1(3):30

[7] Perfilieva I, Daňková M, Bede B (2011) Towards a higher degree F-transform. Fuzzy Sets Syst 180:3-19

[8] Alikhani R, Zeinali M, Bahrami F, Shahmorad S, Perfilieva I (2017) Trigonometric $F^{m}$-transform and its approximative properties. Soft Comput 21:3567-3577

[9] Alikhani R, Ganje MA (2018) An approximation method to solution of boundary value problem by trigonometric fuzzy transform. 14th Seminar on Differential Equations, Dynam. Syst. Appl pp.89-92.

[10] Khastan A, Perfilieva I, Alijani Z (2016) A new fuzzy approximation method to Cauchy problems by fuzzy transform. Fuzzy sets Syst 288:75-95

[11] Zeinali M, Alikhani R, Shahmorad S, Bahrami F, Perfilieva I (2018) On the structural properties of $F^{m}$-transform with applications. Fuzzy Sets Systs 342:32-52

[12] Soltani S, Andersen MS, Hansen PC (2017) Tomographic image reconstruction using training images. Journal of Computational and Applied Mathematics 313:243-258

[13] Bahrami F, Alikhani R, Khastan A (2018) Transport equation with fuzzy data. Iranian J. Fuzzy Syst 15:67-78

[14] Mei JJ, Dong Y, Huang TZ (2019) Simultaneous image fusion and denoising by using fractional-order gradient information. Journal of Computational and Applied Mathematics 351:212-227

[15] Padcharoen A, Kumam P, Martnez-Moreno J (2019) Augmented Lagrangian method for TV-11-12 based colour image restoration. Journal of Computational and Applied Mathematics 354:507-519

[16] Alikhani R, Bahrami F (2019) Fuzzy partial differential equations under the cross product of fuzzy numbers. Information Sciences 494:80-99

[17] Alikhani R, Bahrami F, Parvizi S (2019) Differential calculus of fuzzy multi-variable functions and its applications to fuzzy partial differential equations. Fuzzy Sets Syst 375:100-120

[18] Groza G, Razzaghi M (2019) Approximation of solutions of polynomial partial differential equations in two independent variables. Journal of Computational and Applied Mathematics 346:205-223

[19] Shen S, Liu F, Anh V (2019) The analytical solution and numerical solutions for a two-dimensional multi-term time fractional diffusion and diffusion-wave equation, J. Comput. Appl. Math 345:515-534

[20] McKee S, Vynnycky M, Cuminato JA (2019) An elementary diffusion problem, Laplace transforms and novel mathematical identities. Journal of Computational and Applied Mathematics 353:113-119

[21] Štěpnička M (2008) Fuzzy transform and its application to problems in engineering practice, University of Ostrava, Ostrava, http://irafm. osu. cz/f/PhD-theses/Stepnicka 
[22] Štěpnička M, Valášek R (2004) Fuzzy transforms and their application on wave equation. J. Electr. Eng 55(12):7-10.

[23] Abu-Saman AM, EL-Zerii HS (2016) Fuzzy transform algorithm for partial fractional differential equations. J. Math. Comput. Sci 6(2):235-246

[24] Di Martino F, Sessa S (2019) Complete image fusion method based on fuzzy transforms. Soft Computing 23(6):2113-2123

[25] Manchanda M, Sharma R (2018) An improved multimodal medical image fusion algorithm based on fuzzy transform. Journal of Visual Communication and Image Representation 51:76-94

[26] Hodáková P, Perfilieva I (2013) $F^{1}$-transform of functions of two variables. 8th Conference of the European for Fuzzy Logic and Technology pp.547-553

[27] Di Martino F, Sessa S, Perfilieva I (2017) First order fuzzy transform for images compression. Journal of Signal and Information Processing 8(03):178

[28] Di Martino F, Perfilieva I, Sessa S (2019) A Fast Multilevel Fuzzy Transform Image Compression Method. Axioms 8(4):135

[29] Perfilieva I, Hodáková P, Hurtík P (2016) Differentiation by the F-transform and application to edge detection. Fuzzy Sets Syst 288:96-114

[30] DeVore RA, Lorentz GG (1993) Constructive approximation. Springer, Berlin

[31] Stoer J, Bulirsch R (2002) Introduction to numerical analysis. Springer, New York

[32] Bede B, Rudas IJ (2011) Approximation properties of fuzzy transforms. Fuzzy Sets Syst 180(1):20-40

[33] Di Martino F, Sessa S (2007) Compression and decompression of images with discrete fuzzy transforms. Information Sciences 177(11):2349-2362

[34] Di Martino F, Loia V, Perfilieva I, Sessa S (2008) An image coding/decoding method based on direct and inverse fuzzy transforms. International Journal of Approximate Reasoning 48(1):110-131

[35] Di Martino F, Loia V, Sessa S (2010) Fuzzy transforms for compression and decompression of color videos. Information Sciences 180(20):3914-3931

[36] Perfilieva I, De Baets B (2010) Fuzzy transforms of monotone functions with application to image compression. Information Sciences 180(17):3304-3315 